\title{
ALMA observations of the early stages of substellar formation in the Lupus 1 and 3 molecular clouds
}

\author{
A. Santamaría-Miranda ${ }^{1,2,3}$, I. de Gregorio-Monsalvo ${ }^{1}$, A. L. Plunkett ${ }^{4}$, N. Huélamo ${ }^{5}$, C. López ${ }^{6}$, Á. Ribas ${ }^{1}$, \\ M. R. Schreiber ${ }^{7,3}$, K. Mužić ${ }^{8}$, A. Palau ${ }^{9}$, L. B. G. Knee ${ }^{10}$, A. Bayo ${ }^{2,3}$, F. Comerón ${ }^{11}$, and A. Hales ${ }^{6}$ \\ 1 European Southern Observatory, Av. Alonso de Córdova 3107, Casilla 19001, Santiago, Chile \\ e-mail: asantama@eso.org \\ 2 Instituto de Física y Astronomía, Universidad de Valparaíso, Av. Gran Bretaña 1111, Casilla 5030, Valparaíso, Chile \\ ${ }^{3}$ Núcleo Milenio Formación Planetaria - NPF, Valparaíso, Chile \\ 4 National Radio Astronomy Observatory, 520 Edgemont Rd., Charlottesville, VA 22903, USA \\ 5 Dpto. Astrofísica, Centro de Astrobiología (INTA-CSIC), ESAC Campus, Camino Bajo del Castillo s/n, \\ Urb. Villafranca del Castillo 28692, Villanueva de la Cañada, Spain \\ 6 Joint ALMA Observatory, Av. Alonso de Córdova 3107, Casilla 19001, Santiago, Chile \\ 7 Departamento de Física, Universidad Técnica Federico Santa María, Av. España 1680, Valparaíso, Chile \\ 8 CENTRA, Faculdade de Ciências, Universidade de Lisboa, Ed. C8, Campo Grande 1749-016, Lisboa, Portugal \\ 9 Instituto de Radioastronomía y Astrofísica, Universidad Nacional Autónoma de México, PO Box 3-72, 58090 Morelia, Michoacán, \\ Mexico \\ 10 Herzberg Astronomy and Astrophysics Research Centre, 5071 West Saanich Rd., Victoria, BC V9E 2E7, Canada \\ 11 European Southern Observatory, Karl-Schwarzschild-Strasse 2, 85748 Garching bei München, Germany
}

Received 14 September 2020 / Accepted 17 November 2020

\begin{abstract}
Context. The dominant mechanism leading to the formation of brown dwarfs (BDs) remains uncertain. While the census of Class II analogs in the substellar domain continues to grow, the most direct keys to formation, which are obtained from younger objects (pre$\mathrm{BD}$ cores and proto-BDs), are limited by the very low number statistics available.

Aims. We aim to identify and characterize a set of pre- and proto-BDs as well as Class II BDs in the Lupus 1 and 3 molecular clouds to test their formation mechanism.

Methods. We performed ALMA band $6(1.3 \mathrm{~mm})$ continuum observations of a selection of 64 cores previously identified from AzTEC/ASTE data $(1.1 \mathrm{~mm})$, along with previously known Class II BDs in the Lupus 1 and 3 molecular clouds. Surveyed archival data in the optical and infrared were used to complement these observations. We expect these ALMA observations prove efficient in detecting the youngest sources in these regions, since they probe the frequency domain at which these sources emit most of their radiation.

Results. We detected 19 sources from 15 ALMA fields. Considering all the pointings in our observing setup, the ALMA detection rate was $\sim 23 \%$ and the derived masses of the detected sources were between $\sim 0.18$ and $124 M_{\text {Jup }}$. We classified these sources according to their spectral energy distribution as 5 Class II sources, 2 new Class I/ 0 candidates, and 12 new possible pre-BD or deeply embedded protostellar candidates. We detected a promising candidate for a Class 0/I proto-BD source (ALMA J154229.778-334241.86) and inferred the disk dust mass of a bona fide Class II BD. The pre-BD cores might be the byproduct of an ongoing process of large-scale collapse. The Class II BD disks follow the correlation between disk mass and the mass of the central object that is observed at the low-mass stellar regime.

Conclusions. We conclude that it is highly probable that the sources in the sample are formed as a scaled-down version of low-mass star formation, although disk fragmentation may be responsible for a considerable fraction of BDs.
\end{abstract}

Key words. brown dwarfs - stars: formation - techniques: interferometric - submillimeter: stars

\section{Introduction}

More than two decades have passed since the first brown dwarf (BD) object was discovered (Rebolo et al. 1995; Oppenheimer et al. 1995; Basri et al. 1995). Since then a large number of BDs have been detected in several star-forming regions (SFRs), including Serpens (Lodieu et al. 2002), TaurusAuriga (White \& Basri 2003; Luhman et al. 2018), $\lambda$ Orionis (Barrado y Navascués et al. 2004; Bayo et al. 2011), $\sigma$ Orionis (Caballero et al. 2007; Peña Ramírez et al. 2015) Upper Scorpius (Bouy et al. 2007; Lodieu et al. 2018), $\rho$ Ophiuchi (Alves de Oliveira et al. 2012), NGC 1333 (Scholz et al. 2012), Lupus
(Comerón et al. 2009a; Sanchis et al. 2020), IC 348 in Perseus (Alves de Oliveira et al. 2013), Chamaeleon I (Luhman et al. 2007; Mužić et al. 2015), NGC 2244 (Mužić et al. 2019), and others in the field. However, their formation mechanism(s) are not fully understood.

Several mechanisms have been proposed to explain the formation of BDs, such as an ejection from filaments, disk fragmentation, photoevaporation, eroding outflows, and turbulent fragmentation. The ejection mechanism is based on the fragmentation of a molecular cloud and a subsequent close dynamical interaction between the objects, provoking the ejection of the less massive objects (Reipurth \& Clarke 2001). In this 
picture, Bate et al. (2002) used hydrodynamical simulations and found that $\sim 25 \%$ of the BDs are ejected from the filaments from which they were born, while the rest are formed via disk fragmentation. Hydrodynamical simulations by Bate (2014) showed no statistical difference between the properties of simulated BD and observed BD.

The theory of disk fragmentation suggests that during the formation of planetary-mass companions and substellar companions in a protoplanetary disk, mainly around Sun-like stars, about two thirds of the BDs is expected to be ejected from the disk in the process (Stamatellos \& Whitworth 2009) and those that remain bound to the central star should be able to retain their own disk and accrete. Evidence for accretion in substellar companions on wide orbits ( $>330 \mathrm{AU}$ ) has been found in several objects such as FW Tau B, CT Chab, GSC 06214-210 B, and SR 12 C (Bowler et al. 2014, 2011; Wu et al. 2015; Santamaría-Miranda et al. 2018).

Another BD formation mechanism,namely, photoevaporation, was proposed by Hester et al. (1996) and developed in detail by Whitworth \& Zinnecker (2004). In this scenario, a massive star triggers the formation of a stable pre-stellar core while the ionizing radiation erodes the outside part of the core. Due to the mass loss, the final object is expected to be either a very lowmass star, a BD or a planetary-mass object. Several observational works studying SFRs containing hot stars have identified proto$\mathrm{BD}$ candidates that could have been formed by this mechanism (see e.g., Huélamo et al. 2017; Barrado et al. 2018).

Machida et al. (2009) have proposed that BDs are formed as a scaled-down version of low-mass stars from a core that is less massive and compact with an extreme accretion rate $\left(\sim 10^{-5}-10^{-6} M_{\odot} \mathrm{yr}^{-1}\right)$. This decreases the mass transfer from the envelope to the protostar obtaining a BD as a final object instead of a star. Machida et al. (2008) postulate that the molecular outflow is solely responsible for reducing the mass transfer, lowering the accretion rate and the star formation efficiency (SFE).

Ultimately, there are certain alternative perspectives. For instance, turbulent fragmentation theory (Padoan \& Nordlund 2004; Chabrier et al. 2014) explains that a turbulent environment driven by supersonic turbulence can naturally yield the formation of substellar fragments, which should be gravitationally unstable and collapse to form pre- and proto-BDs. On the other hand, the Global Hierarchical Collapse scenario (VázquezSemadeni et al. 2019) proposes that Hoyle-like gravitational fragmentation (Hoyle 1953) in the presence of low levels of turbulence can continue down to substellar-mass fragments, which finally end up in BDs. In both cases, it is expected that BDs are formed as a scaled-down version of low-mass stars.

One of the main goals in the field of the formation of substellar objects is to test the different formation mechanisms and to determine which of them is dominant. If BDs are formed as a scaled-down version of low-mass stars, they should have cold envelopes and outflows similar to those observed in Class 0/I protostars. To test this scenario, we need to increase the number of detected sources at the earliest evolutionary stages in the (sub)millimetre $(\mathrm{mm})$ regime when they are still embedded in the parental cloud - namely, the pre- and proto-BDs. So far, the only confirmed pre-BD is Oph B-11 (André et al. 2012) and there are pre-BD candidates both in Taurus (Palau et al. 2012; Tokuda et al. 2019) and Barnard 30 (Huélamo et al. 2017; Barrado et al. 2018). Regarding proto-BDs, one excellent Class 0/I candidate has been identified in Perseus (Palau et al. 2014) and other candidates are proposed in Taurus (Apai et al. 2005; Barrado et al. 2009; Palau et al. 2012; Morata et al. 2015;
Dang-Duc et al. 2016), Chamaeleon II (de Gregorio-Monsalvo et al. 2016), Ophiuchus (Whelan et al. 2018; Riaz et al. 2018; Kawabe et al. 2018), Serpens (Riaz et al. 2016, 2018), and $\sigma$ Orionis (Riaz et al. 2015, 2017, 2019), although many of these may also be more evolved Class I objects. Finally, the search and study of very low luminosity objects (VeLLOs) have revealed sources that show proto-BD characteristics (Bourke et al. 2006; Lee et al. 2009, 2013, 2018; Kauffmann et al. 2011; Kim et al. 2019; also see the references in Table 4 of Palau et al. 2014).

Class II BDs are also expected to exhibit phenomena similar to those seen in very low mass (VLM) protostars, including disks and jets in the optical and infrared. Indeed, circumstellar disks around BDs were identified by Natta \& Testi (2001) and Natta et al. (2002) through the study of spectral energy distributions (SEDs) and the confirmed presence of jets, Herbig-Haro objects, outflows, and accretion (Jayawardhana et al. 2003; Natta et al. 2004; Whelan et al. 2005; Phan-Bao et al. 2008; Riaz et al. 2017). In particular, (sub)millimetre observations are important to study the properties of BD disks. The first (sub)mm study was presented by Klein et al. (2003), who reported the detection of millimetre dust emission from BDs. Then, Scholz et al. (2006) in a sample of 20 BD disks in Taurus inferred dust settling towards the disk midplane. Following these pioneering works, BD disks were observed using ALMA, constraining their main parameters, such as the dust and gas disk masses or their radii, which provide information about the formation of these objects. The first ALMA study of three BD disks was performed in Taurus (Ricci et al. 2014). Subsequent studies (Testi et al. 2016) showed the possible environmental influence on the formation of BDs in Ophiuchus when compared to Taurus disks. A similar survey in Upper Scorpius (van der Plas et al. 2016) revealed a new relation between the stellar luminosity and the temperature in the substellar regime. The TBOSS survey with ALMA (Ward-Duong et al. 2018) included BD disks in Taurus, and IC 348, and Chamaeleon that have been studied by Ruíz-Rodríguez et al. (2018) and Pascucci et al. (2016). Finally, Lupus BDs have been also observed with ALMA (Ansdell et al. 2016, 2018; Sanchis et al. 2020).

The Soul of Lupus with ALMA Consortium (SOLA, Saito et al. 2015) is focused on the study of the Lupus clouds. The Lupus molecular complex (Barnard 1927) is composed of nine molecular clouds. In this work, we focus on the Lupus 1 and 3 clouds. Despite both clouds belonging to the same complex, they show different properties. The mass of Lupus 1 is an order of magnitude larger than that of Lupus 3. Cambrésy (1999) derived a mass $2.6 \times 10^{4} M_{\odot}$ for Lupus 1 and $3 \times 10^{3} M_{\odot}$ for Lupus 3 from extinction values; a similar mass ratio was obtained using ${ }^{13} \mathrm{CO}$ (Tachihara et al. 1996) with masses of $1.3 \times 10^{3} M_{\odot}$ and $3.4 \times 10^{2} M_{\odot}$, respectively. Lupus 1 is more isolated in the complex than is Lupus 3 , the latter of which is surrounded by Lupus clouds 2, 4, 5, and 6. (Comerón 2008, and references therein). The stellar populations are also different: Lupus 1 is dominated by early M dwarfs (Comerón et al. 2009a) and Lupus 3 is abundant in $\mathrm{T}$ Tauri stars. Furthermore, Lupus 3 has the highest star formation rate and column density of all the Lupus clouds (Comerón 2008, and references therein).

In this work, we present the main results from the SOLA project. The objective is to shed light on the main formation mechanism of BDs. We have complemented the ALMA data with optical and infrared archival observations to build the SED of all the objects. In Sect. 2, we present the AzTEC and ALMA observations performed for this SOLA project. In Sect. 3, we present the data analysis, including ALMA continuum 
detections, mass estimates, the optical/infrared counterpart association to the ALMA detections, SEDs, bolometric temperatures and luminosities, and a brief description of each source. In Sect. 4, we present a comparison of our results with previous AzTEC results and assess them against the main BD formation theories. We also discuss the detection rate and the evolution of the cores and compare the disk masses with those in other SFRs. Finally, our conclusions and summary are presented in Sect. 5.

\section{Observations}

\subsection{The sample}

The sample is based on two maps of Lupus 1 and 3 clouds (Fig. 1), taken with the AzTEC $1.1 \mathrm{~mm}$ array camera at the Atacama Submillimeter Telescope Experiment (ASTE) telescope located in the Atacama Desert in northern Chile. A subset of these data were presented by Tsukagoshi et al. (2011) and Tamura et al. (2015), who studied V1094 Sco (discussed later in this work) and bright submillimetre galaxies, respectively.

The SOLA Consortium was given access to these two maps of millimetre continuum observations and in using them, we have revealed hundreds of continuum sources embedded within filamentary structures. The angular resolution of the AzTEC observations was $28^{\prime \prime}$ with a FoV of $7.8^{\prime}$ and a rms noise level of $5 \mathrm{mJy} \mathrm{beam}^{-1}$. More information about these observations is found in Tsukagoshi et al. (2011) and Tamura et al. (2015).

To select dust cores in AzTEC maps, we first used the clumpfind algorithm (Williams et al. 1994). As part of the selection criteria for our source sample, we selected AzTEC clumps with peak intensities in the range $30-100 \mathrm{mJy}$, which show a mass below $0.225 M_{\odot}$ assuming a temperature of $10 \mathrm{~K}$ and an emissivity index of $\beta=2$. Considering a SFE $30 \%$ (Motte et al. 1998) these sources should form substellar objects. Using this methodology, we obtained 39 AzTEC cores that were not previously reported in the literature.

Then, we cross-correlated the AzTEC substellar cores with optical and infrared archival observations (see Sect. 2.2) to build the SEDs. The SEDs were consistent with young (sub)stellar objects (YSO). These cores were classified as 33 pre-BD objects along with 6 Class 0 and I objects. A detailed explanation of classification methodology used in this work using the SED is in Sect. 3.3.2.

We complemented the AzTEC sample with previously known substellar objects in Lupus 1 and 3 in order to fully characterize the substellar population at different evolutionary stages. We selected 22 already known Class II BD and 3 Class I/II BD from Merín et al. (2008), Comerón et al. (2009a), Mužić et al. (2014).

In summary, the final sample of 64 objects was divided in a preliminary classification of 33 pre-BD objects, 6 Class 0 and I objects, 3 Class I/II, and 22 Class II objects. The initial classification, phase centre coordinates, and noise rms are given in Table A.1.

\subsection{Ancillary data}

The combination of the AzTEC maps and a subsequent search of a catalog derived from literature and archival data (López et al., in prep.) enabled us to obtain a set of good-quality preand proto-BDs candidates. This catalog includes data from optical to the (sub)millimetre wavelengths. In order to classify the objects detected with ALMA (see Fig. 2 to see the position of the detected sources in a Herschel map), we searched in the archives of several observatories. The filters used and the respective astrometric accuracy for each telescope/instrument are given in Table 1.

Optical data were obtained from Comerón et al. (2009a) using the Wide Field Imager (WFI) at the $2.2 \mathrm{~m} \mathrm{MPG/ESO} \mathrm{Tele-}$ scope (Baade et al. 1999).

The near-infrared wavelength range data was covered by two surveys: the Deep Near Infrared Survey of the Southern Sky (DENIS) at the $1 \mathrm{~m}$ ESO telescope (Epchtein et al. 1994) and the Cerro Tololo Inter-American Observatory 2MASS telescope (CTIO/2MASS; Skrutskie et al. 2006).

Mid-infrared wavelength data were obtained from the WideField Infrared Survey Explorer (WISE, Wright et al. 2010 and the InfraRed Array Camera on the Spitzer Space Telescope (IRAC; Fadda et al. 2004), both NASA space missions. Spitzer had another instrument, the Multiband Imaging Photometer for SIRTF(MIPS), that provided far-infrared wavelength coverage. Additionally, two more space telescopes provided farinfrared data: ASTRO-F (Murakami et al. 2007) from JAXA and Herschel (Pilbratt et al. 2010) from the European Space Agency (ESA). From Herschel we obtained data from two instruments: the Photodetector Array Camera and Spectrometer (PACS; Poglitsch et al. 2010) and the Spectral and Photometric Imaging Receiver (SPIRE; Griffin et al. 2010).

Millimetre and submillimetre observations of Lupus 3 were also available from the Large Apex BOlometer CAmera (LABOCA, Siringo et al. 2009) at the APEX Observatory.

Finally, data from other telescopes including the Australia Telescope Compact Array (ATCA), the Swedish-ESO Submillimetre Telescope (SEST), and the Submillimeter Array (SMA) were examined, but no detections were obtained from any of these telescopes for Lupus 1 and 3.

\subsection{ALMA observations}

ALMA observations were performed between 31 March and 1 April 2016 as part of the ALMA Cycle 3 program 2015.1.00512.S. We observed all 64 targets in our sample using single field interferometry in Band 6, with a field of view (FoV) of 23". The number of antennas for each data set ranged between 42 and 44 . The total allocated time for this program was $6.7 \mathrm{~h}$ including overheads, with an average time on each science source of $4.7 \mathrm{~min}$. Data were taken under good and stable weather conditions (precipitable water vapour 1.1-1.7 mm).

The correlator was set up to observe five different spectral windows in dual polarization mode with the primary aim of detecting continuum emission, while also detecting possible molecular emission lines. One of the basebands was configured to observe two spectral windows of $0.469 \mathrm{GHz}$ bandwidth and $0.488 \mathrm{MHz}$ channel width $\left(\sim 0.3 \mathrm{~km} \mathrm{~s}^{-1}\right.$ velocity resolution) each, one of them centred at $230.538 \mathrm{GHz}, \mathrm{CO}(2-1)$ rest frequency, and the other one at $231.150 \mathrm{GHz}$. The other three basebands were configured to observe three different spectral windows of $1.875 \mathrm{GHz}$ each, and at a spectral resolution of $1.938 \mathrm{MHz}\left(\sim 2.5 \mathrm{~km} \mathrm{~s}^{-1}\right.$ velocity resolution) centred at 233.5 GHz, 217.0 GHz and $219.25 \mathrm{GHz}$ for possible detections of $\mathrm{C}^{18} \mathrm{O}(2-1), \mathrm{SiO}(5-4)$, and $\mathrm{DCN}(3-2)$ and to have the best atmospheric transmission.

QSOJ1517-2422 was used as bandpass calibrator for the whole sample. This QSO was used as a flux calibrator for one of the datasets while Titan was used for the remaining execution blocks. Two phase calibrators were used: for the three datasets of Lupus 1 QSO J1610-3958 was used, while QSO J1534-3526 was used for the observations towards Lupus 3. 


\section{Lupus 1}

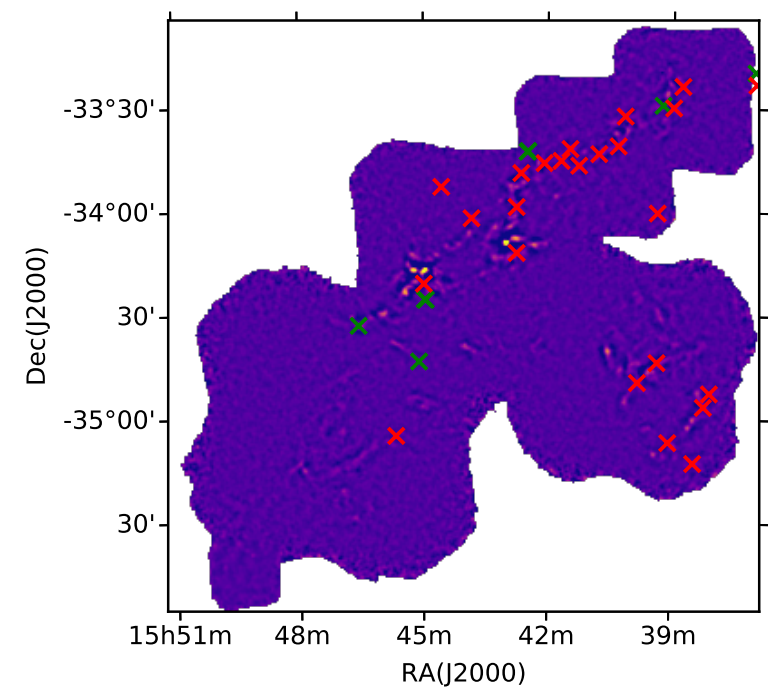

Lupus 3

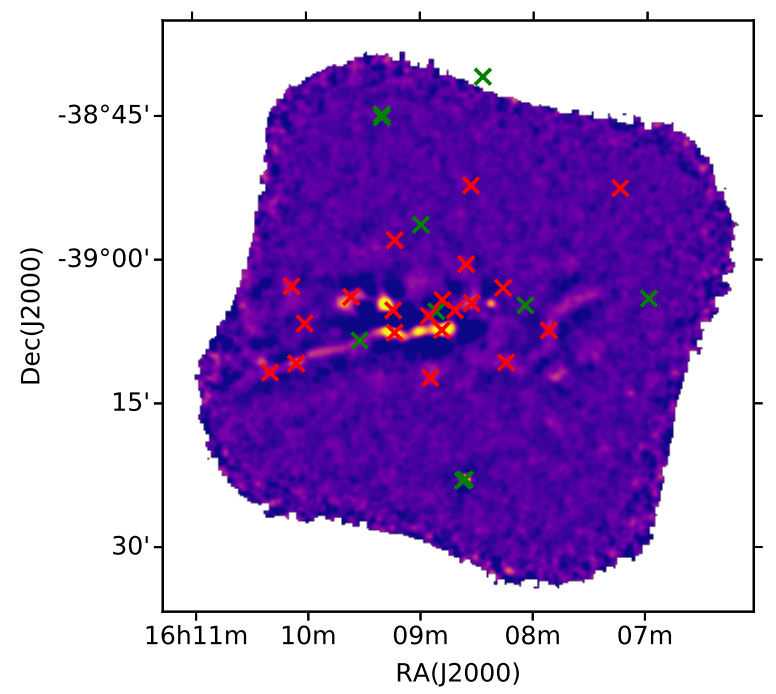

Fig. 1. AzTEC $1.1 \mathrm{~mm}$ maps of Lupus 1 (left panel) and Lupus 3 molecular clouds (right panel) overlaid with the ALMA pointings. Green crosses represent ALMA detections and red ones the AzTEC-identified sources that were not detected with ALMA.
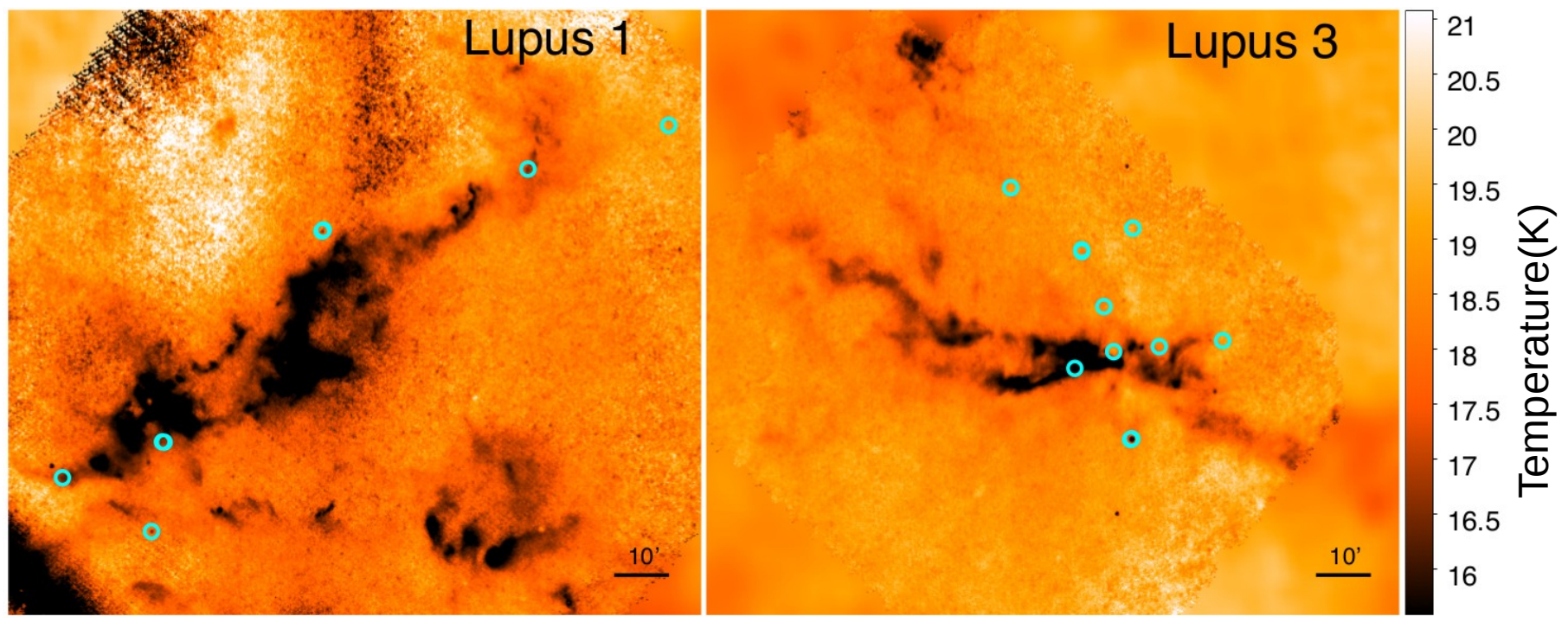

Fig. 2. Herschel temperature maps for Lupus 1 and 3 clouds (Teixeira et al., in prep.). North is up and East to the left. The ALMA detections are displayed as cyan circles and some circles overlap.

Table 1. Telescopes and filters for archival data.

\begin{tabular}{lccccc}
\hline \hline $\begin{array}{l}\text { Telescope/ } \\
\text { instrument or survey }\end{array}$ & Filter $_{1}\left(\lambda_{1}\right)$ & Filter $_{2}\left(\lambda_{2}\right)$ & Filter $_{3}\left(\lambda_{3}\right)$ & Filter $_{4}\left(\lambda_{4}\right)$ & $\begin{array}{c}\text { Astrometric accuracy } \\
\text { [arcsec] }\end{array}$ \\
\hline ESO 2.2 m/WFI & $R_{c}(657.1 \mathrm{~nm})$ & $I_{c}(826.9 \mathrm{~nm})$ & $Z(964.8 \mathrm{~nm})$ & & $\sim 2$ \\
ESO 1 m/DENIS & $I(0.82 \mu \mathrm{m})$ & $J(1.25 \mu \mathrm{m})$ & $K(2.15 \mu \mathrm{m})$ & & 0.1 \\
CTIO/2MASS & $J(1.235 \mu \mathrm{m})$ & $H(1.662 \mu \mathrm{m})$ & $K_{s}(2.159 \mu \mathrm{m})$ & & 0.50 \\
WISE & $W 1(3.4 \mu \mathrm{m})$ & $W 2(4.6 \mu \mathrm{m})$ & $\mathrm{W} 3(12 \mu \mathrm{m})$ & $W 4(22 \mu \mathrm{m})$ & $0.25-0.50$ \\
Spitzer/IRAC & $1(3.6 \mu \mathrm{m})$ & $2(4.5 \mu \mathrm{m})$ & $3(5.8 \mu \mathrm{m})$ & $4(8.0 \mu \mathrm{m})$ & $\sim 0.5$ \\
Spitzer/MIPS & $1(23.675 \mu \mathrm{m})$ & $2(71.42 \mu \mathrm{m})$ & $3(155.9 \mu \mathrm{m})$ & & 1.6 \\
Akari & $\mathrm{f}(9 \mu \mathrm{m})$ & $\mathrm{f} 18(18 \mu \mathrm{m})$ & & & 1.6 \\
Herschel/PACS & $70 \mu \mathrm{m}$ & $100 \mu \mathrm{m}$ & $160 \mu \mathrm{m}$ & & 4.5 \\
Herschel/SPIRE & $250 \mu \mathrm{m}$ & $350 \mu \mathrm{m}$ & $500 \mu \mathrm{m}$ & & 2 \\
APEX/LABOCA & $868.9 \mu \mathrm{m}$ & & & & \\
AzTEC/ASTE & $1.1 \mathrm{~mm}$ & & & & \\
\hline
\end{tabular}


The Common Astronomy Software Applications package (CASA; McMullin et al. 2007) was used to process the data. Most of the datasets used the pipeline version 4.5.3 for calibration and image processing and only one of the executions was reduced using the ALMA standard calibration mode (not pipelined) in CASA version 4.6. The task CLEAN was used to produce continuum and spectral line images. We selected a Briggs weighting with Robust parameter value of 2 to produce all the images. Primary beam correction was applied before inferring physical parameters from the images. The achieved noise rms at the phase centre was on average $\sim 70 \mu \mathrm{Jy} \mathrm{beam}^{-1}$ for the continuum and $\sim 6 \mathrm{mJy}_{\text {beam }}^{-1}$ in a $0.3 \mathrm{~km} \mathrm{~s}^{-1}$ channel width for the spectral line data. The angular resolution of the images was $\sim 0.9^{\prime \prime}$, corresponding to $\sim 138 \mathrm{AU}$ at $154 \mathrm{pc}$ (see Sect. 2.4). The absolute positional accuracy of the ALMA images in our sample spans between 4 and 270 mas with an average value of 148 mas $^{1}$.

We detected 19 sources from the 15 ALMA fields containing 64 science targets. Detailed information for each source detection is given in Table 2. The main properties of the detected sources are described in Sect. 3.

We adopted the naming convention recommended by ALMA for newly discovered sources, but for convenience in the discussion we shorten the source names to J\#\#\#\#\#, for example ALMA J154228.675-334230.18 is referred to as J154228, with a minor modification in the case of ALMA J160920.089-384515.92 (J1609200) and ALMA J160920.171-384456.40 (J1609201) in order to distinguish them.

\subsection{Distances}

We used the parallaxes from Gaia Data Release 2 (Gaia Collaboration 2018) to obtain an accurate average distance estimate of the Lupus 1 and 3 clouds. We used a sample of spectroscopically confirmed members (Comerón et al. 2009a) of Lupus 1 and 3 and applied the kalkayot ${ }^{2}$ python code (Olivares et al. 2020) that uses a Bayesian approach to obtain a precise distance, as recommended in Bailer-Jones (2015). We also used this methodology to obtain the distance for single sources in both Lupus 1 and 3 when their parallaxes were available (see Table 3 ). Otherwise we assumed the mean distance for each cloud that we obtained,namely, $153.4 \pm 4.6$ and $154.8 \pm 9.6 \mathrm{pc}$ for Lupus 1 and 3, respectively. There are two Class II BDs (153701.1-332 and 160545.8-385) in our survey whose distances are not the expected ones for Lupus 1 and 3 using $3 \sigma$. Therefore, we do not consider these sources as part of the complex. In order to make comparisons among source characteristics in Lupus and other SFRs in the literature, we recalculated distances to the regions that we will reference in Sect. 4.6. Chamaeleon I distances for individual sources are included in Manara et al. (2019). For the sources without Gaia parallaxes, we assumed the distance to Chamaeleon I cloud, $179_{-10}^{+11}$ (Voirin et al. 2018). Upper Scorpius distances for individual sources and the mean distance of the cloud are included in Galli et al. (2018); we assumed the mean distance to the cloud for the sources without Gaia parallaxes. Taurus distances are included in Galli et al. (2019). In Ophiuchus, we derived the distances from Gaia DR2 parallaxes

\footnotetext{
1 ALMA Technical Handbook, Chapter 10.5.2 Astrometric Observations, https://help.almascience.org/index.php?/ Knowledgebase/Article/View/319/6/what-is-theastrometric-accuracy-of-alma

2 https://github.com/olivares-j/Kalkayotl
}

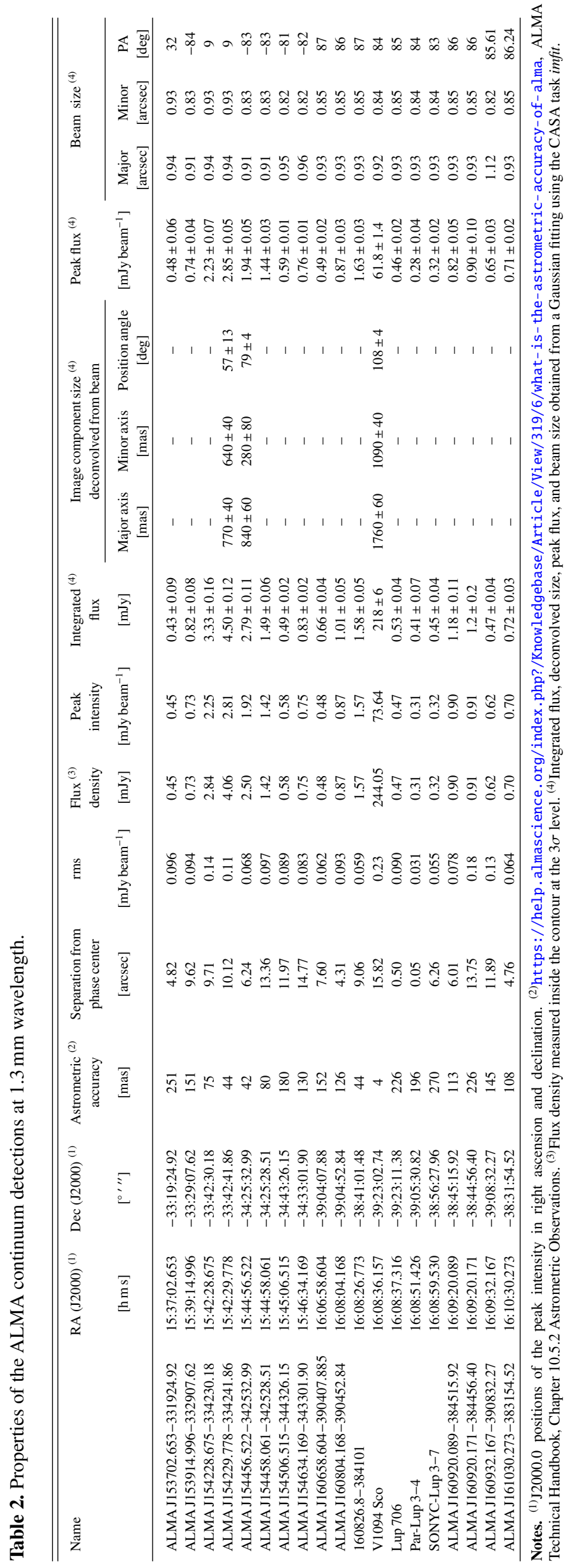

A10, page 5 of 30 
Table 3. Distances using Gaia Data Release 2.

\begin{tabular}{lc}
\hline \hline Name & Distance [pc] \\
\hline \multicolumn{2}{c}{ Lupus BDs detected with ALMA } \\
\hline 160826.8-384101 & $165 \pm 4$ \\
V1094 Sco & $154.7 \pm 1.1$ \\
Lup 706 & $191 \pm 29$ \\
Par-Lup 3-4 & $155 \pm 14$ \\
SONYC-Lup 3-7 & $151 \pm 6$ \\
\hline Lupus BDs not detected with ALMA \\
\hline 153701.1-332255 & $94 \pm 4$ \\
153709.9-330129 & $175 \pm 7$ \\
153921.8-340020 & $152 \pm 9$ \\
154140.8-334519 & $151 \pm 8$ \\
160545.8-385454 & $44 \pm 1$ \\
160714.0-385238 & $125 \pm 4$ \\
160816.0-390304 & $164 \pm 5$ \\
160833.0-385222 & $158 \pm 4$ \\
160848.2-390920 & $184 \pm 5$ \\
161144.9-383245 & $169 \pm 5$ \\
161225.6-381742 & $159 \pm 3$ \\
161210.4-390904 & $233 \pm 30$ \\
\hline \multicolumn{2}{c}{ Ophiuchus } \\
\hline ISO-Oph030 \\
ISO-Oph032 & $137 \pm 7$ \\
ISO-Oph042 & $150 \pm 9$ \\
ISO-Oph102 & $154 \pm 19$ \\
GY92-264 & $142 \pm 6$ \\
ISO-Oph160 & $139 \pm 5$ \\
ISO-Oph164 & $142 \pm 22$ \\
GY92-320 & $142 \pm 16$ \\
ISO-Oph176 & $141 \pm 18$ \\
ISO-Oph193 & $140 \pm 19$ \\
\hline \multicolumn{2}{c}{$147 \pm 17$} \\
\hline \multicolumn{2}{c}{. } \\
153
\end{tabular}

(Gaia Collaboration 2018) using the mentioned kalkayotl code. Using a selection of confirmed members (Natta et al. 2006) in the Ophiuchus SFR, we obtained an average distance to Ophiuchus SFR of $139.26 \pm 14.57 \mathrm{pc}$. The distance for individual sources in Ophiuchus using the same methodology are in Table 3. The distance to Lupus sources in Sanchis et al. (2020) that were not part of our sample are included in Manara et al. (2019).

\section{Results}

\subsection{ALMA: continuum detections}

The ALMA continuum images for each detection are shown in Fig. 3. In total, 19 sources were detected in continuum emission in 15 pointings among the 64 science targets. The flux density for detections ranged between 0.22 and 244.05 mJy (see Table 2).

There are four ALMA pointings where we detect two sources inside the primary beam (J154228 and J154229, J154456 and J154458, J1609200 and J160920171, Lup 706 and V1094 Sco) as can be seen in Fig. B.1. Finally, we note that most of the detected sources (16 out of 19) are spatially unresolved in our observations.

All the sources detected in the continuum with ALMA are included in Table 2. We provide the position, the separation from the phase centre, the rms, the flux density, and the peak intensity. Other quantities such as the integrated flux, size, peak flux, and beam size obtained from a Gaussian fitting using the task imfit in CASA are also included in the table.

\subsection{ALMA: Gas emission detections}

Although obtaining continuum emission images was the main observational goal of the ALMA observations, the $\mathrm{CO}(2-1)$ emission line was also included in the spectral setup. Detecting gas emission close to the cloud velocity for our sources would be a confirmation of their cloud association. Unfortunately, we only have a clear gas detection in two sources. The first one is V1094 Sco and it was studied by Ansdell et al. (2016, 2018). The second one is Par-Lup 3-4 where we discovered the base of a bipolar molecular outflow traced by CO gas (Santamaría-Miranda et al. 2020). Extended cloud emission is seen in ${ }^{12} \mathrm{CO}(2-1)$ surrounding $\mathrm{J} 154634$ at velocities between +3.4 and $+5.3 \mathrm{~km} \mathrm{~s}^{-1}$, but no spatially compact gas emission was detected at the position of this source. These velocities are consistent with those of the Lupus 3 cloud, $+4.1 \mathrm{~km} \mathrm{~s}^{-1}$ with $\Delta V=1.7 \mathrm{~km} \mathrm{~s}^{-1}$ (Tachihara et al. 1996). We note that several disks in Lupus 3 show systemic velocities in that range (e.g., EX Lup at $+4.4 \mathrm{~km} \mathrm{~s}^{-1}$ Hales et al. 2018 or the sources in Ansdell et al. 2018). The rest of the ALMA sources were not detected in $\mathrm{CO}$ and have an upper limit to their $\mathrm{CO}(2-1)$ fluxes of $\sim 6 \mathrm{mJy} \mathrm{beam}^{-1}(1 \sigma)$ in a $0.3 \mathrm{~km} \mathrm{~s}^{-1}$ channel. Additionally, there is no detection of the other emission lines $\left(\mathrm{C}^{18} \mathrm{O}(2-1), \mathrm{SiO}(5-4)\right)$ in any of the sources with a rms of $\sim 4 \mathrm{mJy}$ beam $^{-1}, \sim 1 \mathrm{mJy}^{-1}$ beam $^{-1}$, respectively, with both of them in a $2.7 \mathrm{~km} \mathrm{~s}^{-1}$ channel.

\subsection{Spectral energy distribution}

\subsubsection{Optical/IR/radio counterpart association}

To associate the ALMA detections with optical/IR/radio sources, we first inspected the archival images. A total of seven counterpart sources were identified by searching within a separation smaller than the total accuracy error. The total accuracy error is calculated as the square root of the quadratic sum of ALMA astrometric accuracy and the astrometric accuracies of the counterpart's instrument. In the case of the ESO $2.2 \mathrm{~m} / \mathrm{WFI}$, for which there is no astrometric accuracy, we use a conservative value of $\sim 2^{\prime \prime}$. The astrometric accuracies were between 1 and 2 arcsec, depending on the instrument, and are listed in Table 1. The SEDs of all the ALMA detections are consistent with YSOs and are displayed in Fig. 4.

\subsubsection{Classification}

We classified the sources in the following categories: ALMA detections without optical/IR counterpart (pre-BD phase), Class 0, Class I, and Class II sources. For this classification, we use the $\alpha_{\text {IR }}$ slope (Adams et al. 1987), as well as the $T_{\text {bol }}$ and $L_{\mathrm{bol}}$ values. We used the $\alpha_{\mathrm{IR}}$ slope values in Greene et al. (1994) where Class 0 protostars have $\alpha_{\mathrm{IR}}$ slope values exceding 0.3 , Class I/II sources have $\alpha_{\mathrm{IR}}$ slope values between -0.3 and 0.3 , Class II sources have $\alpha_{\text {IR }}$ slope values between -1.6 and -0.3 , and Class III sources have $\alpha_{\mathrm{IR}}$ slope values below -1.6 . We used different combinations of photometric bands $(2.16 \mu \mathrm{m}$, $12 \mu \mathrm{m}),(2.16 \mu \mathrm{m}, 23.67 \mu \mathrm{m})$ and $(3.4 \mu \mathrm{m}, 23.67 \mu \mathrm{m})$ because not all the photometry was available for all sources.

We calculated the bolometric luminosity $\left(L_{\mathrm{bol}}\right)$ and the bolometric temperature $\left(T_{\text {bol }}\right)$ for each source that has at least a counterpart at three different wavelengths (see Table 4). This group 
A. Santamaría-Miranda et al.: Early stages of substellar formation in the Lupus 1 and 3 molecular clouds
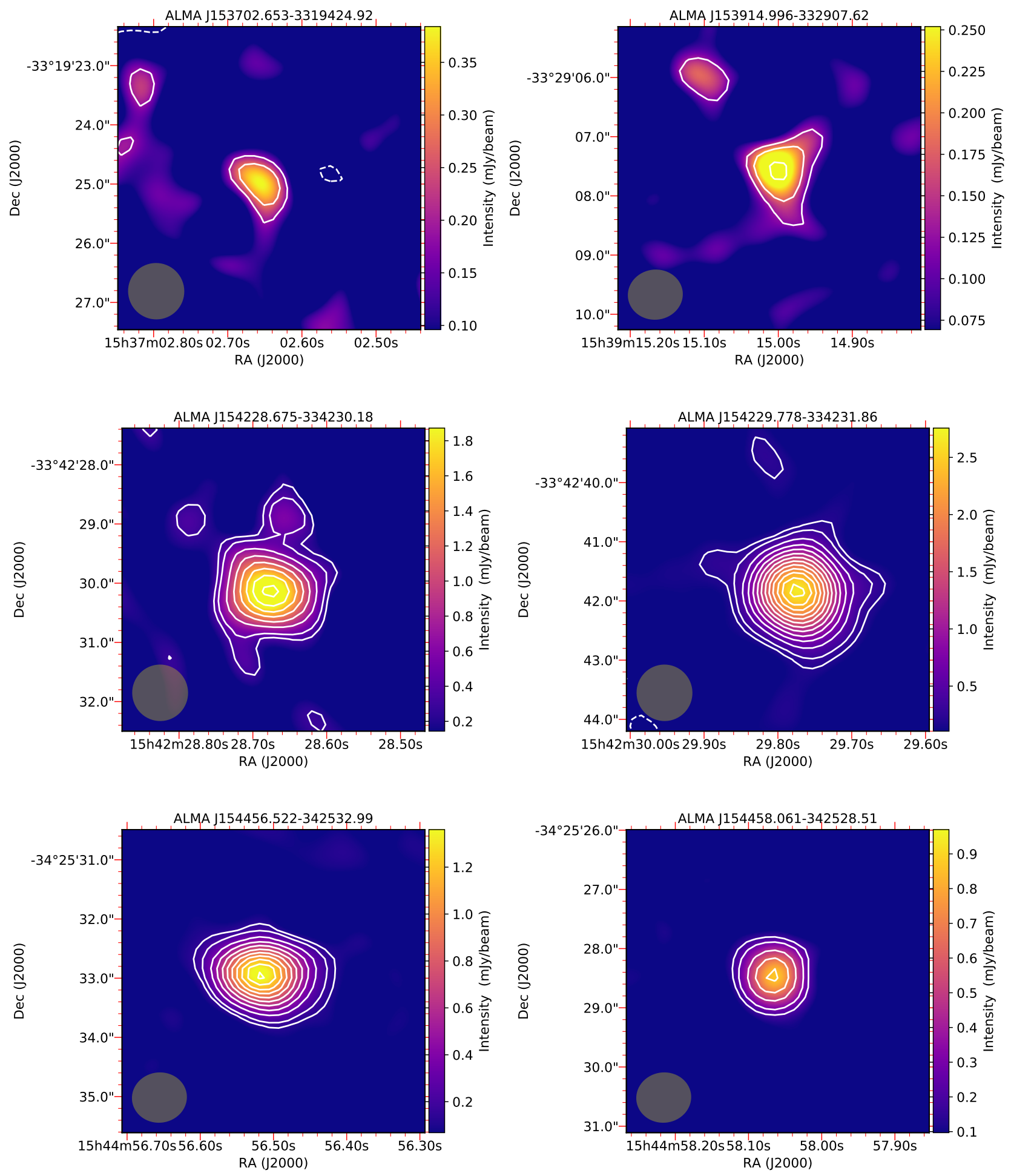

Fig. 3. $1.3 \mathrm{~mm}$ ALMA continuum images of the 19 detected sources. White contours are 2, 3, 5, 7, 9, 11, 13, 15, 17, 19, 21, 23, 25, 27, 29, 50, $100,150 \sigma$. Here, $\sigma$ is the rms noise level of each respective map, given in Table 2. Dashed contours are negative emission at $-2 \sigma$. Beam size is represented by the grey ellipse in the bottom left corner. Continued on Fig. B.2.

of sources includes: J154229, 160826, V1094 Sco, Lup 706, Par-Lup 3-4, and SONYC-Lup 3-7. We also included J153914 to obtain a lower limit, although this source does not have an infrared counterpart. To calculate $L_{\mathrm{bol}}$, we used formula (1) from Enoch et al. (2009), and for $T_{\text {bol }}$, we used formula (2) from the same paper and the mean frequency from Myers \& Ladd (1993). In addition, to classify Class 0 , I or II/III consistently, we used the definition in Chen et al. (1995) where Class 0 protostars have $T_{\text {bol }}$ below $70 \mathrm{~K}$, Class I sources have temperatures between 70 and $650 \mathrm{~K}$, and Class II-III sources temperatures exceeding $650 \mathrm{~K}$.

One source $(\mathrm{J} 154229)$ is at the boundary between Class 0 and Class I objects, according to its $T_{\text {bol }}$ value. Using the $T_{\text {bol }}$ classification method Par-Lup 3-4 and Lup 706 appear to be 

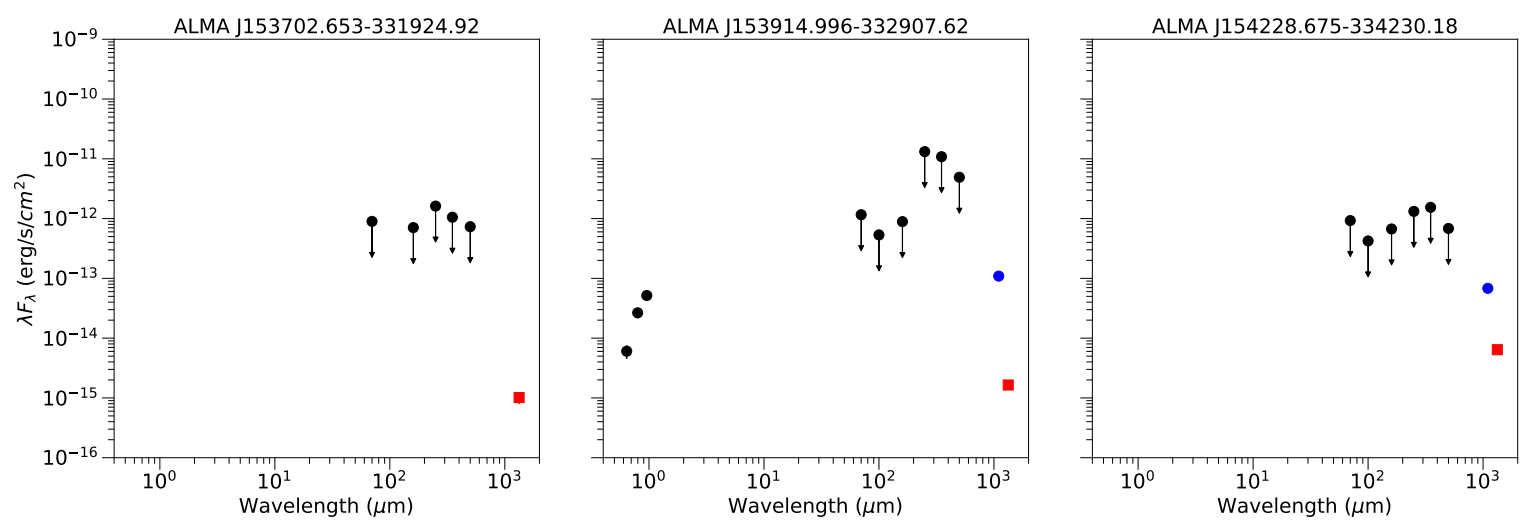

Fig. 4. SED for sources detected with ALMA at $1.3 \mathrm{~mm}$. Red squares show the ALMA fluxes. Blue circles show the AzTEC fluxes. Black circles show the fluxes from other telescopes: WFI, DENIS, 2MASS, WISE, Spitzer, Akari, Herschel, and APEX/LABOCA. Upper limits are included as arrows. Continued on Fig. B.3.

Table 4. Bolometric temperature and bolometric luminosity for those ALMA detections with more than three points in their SED.

\begin{tabular}{lcc}
\hline \hline Name & $\begin{array}{c}\text { Temperature } \\
{[\mathrm{K}]}\end{array}$ & $\begin{array}{c}\text { Luminosity } \\
{\left[L_{\odot}\right]}\end{array}$ \\
\hline $\mathrm{J} 154229$ & $64.6_{-1.7}^{+2.4}$ & $0.0044 \pm 0.0008$ \\
$\mathrm{~J} 153914$ & $>46.92$ & $>0.04012$ \\
160826 & $1411_{-5}^{+9}$ & $0.0268_{-0.0005}^{+0.0009}$ \\
V1094 Sco & $2019_{-139}^{+25}$ & $0.5600_{-0.0005}^{+0.0009}$ \\
Lup 706 & $415_{-14}^{+10}$ & $0.0290 \pm 0.003$ \\
Par-Lup 3-4 & $406_{-24}^{+21}$ & $0.0190 \pm 0.0006$ \\
SONYC-Lup 3-7 & $1959_{-14}^{+16}$ & $0.0095 \pm 0.0004$ \\
\hline
\end{tabular}

Class I objects, although both sources have been previously classified as Class II in the literature. These two sources are mentioned in Alcalá et al. (2014) as having the lowest luminosities among all the Lupus YSOs. The subluminous nature of ParLup 3-4 has been investigated by Huélamo et al. (2010) concluding that it could be explained by the presence of a close to edge-on disk (inclination of $\sim 81^{\circ}$ ). A detailed study of this source has been presented by Santamaría-Miranda et al. (2020), confirming the high inclination of the system. In the case of Lup706, the $\alpha_{\mathrm{IR}}$ slope shows values of Class I/II or Class II using different combination of photometric bands, it may be in transition to Class I to Class II. The remaining sources (160826, V1094 Sco, and SONYC-Lup 3-7) can be classified as Class II or III objects and we maintain their previous classifications. Figure 5 shows the position in the $T_{\text {bol }}-L_{\text {bol }}$ diagram of the mentioned sources along with objects from previous studies in the regions of Perseus and Taurus.

The sources we study in this work have lower luminosities than other BD candidates from previous works, as shown in Fig. 5, with the exception of the proto-BD candidate J041757 of Barrado et al. (2009) and Palau et al. (2012). Thanks to the sensitivity of ALMA and the close proximity of the Lupus complex, we have detected the least luminous pre-BD candidate so far (J160658). We also detected a new Class 0/I substellar candidate (J154229), a type of object for which confirmed detections are very few in number (e.g., ICM348-SSM2E, Palau et al. 2014). Class I sources are also difficult to detect, and here we add one new candidate to the list of Class I substellar candidate sources such as L1148-IRS (Kauffmann et al. 2011), and J042118 and
J041757 (Palau et al. 2012). The ALMA detection of the Class II sources provides a new point in the SED that helps us to better constrain their position in the $T_{\text {bol }}-L_{\text {bol }}$ diagram.

Additionally, we identified the whole sample as VeLLOs, with the exception of V1094 Sco. These VeLLOs are sources that have an internal luminosity $\left(L_{\text {int }}\right)$ below $0.1 L_{\odot}$. To estimate $L_{\text {int }}$, we used the formula from Dunham et al. (2008):

$L_{\text {int }}\left(L_{\odot}\right)=3.3 \times 10^{8}\left(F_{70}\left(\frac{d}{140}\right)^{2}\right)^{0.94}$

where $F_{70}$ is the flux at $70 \mu \mathrm{m}$. For almost all the objects in our sample we only have upper limits (see Table C.1) for that specific wavelength. Using these upper limits we find that all our sources are below the VeLLO luminosity threshold.

Finally, we double-checked previous classifications in the literature, if available, for all the sources. Table 5 shows the classification for each source using the different methods.

\subsection{ALMA: Mass estimates}

The total mass of the ALMA detections (see Table 6) is calculated assuming that the observed emission is optically thin and the gas-to-dust mass ratio is 100 (Bohlin et al. 1978). Given these assumptions, we use the following formula from Hildebrand (1983) to estimate the dust and gas mass:

$M=\frac{S_{v} D^{2}}{B_{v}\left(T_{\mathrm{d}}\right) \kappa_{v}}$.

Here $S_{v}$ is the flux density in the region inside a $3 \sigma$ contour level, $D$ is the distance to the source, or the average cloud distance if not in Table $3, B_{v}\left(T_{\mathrm{d}}\right)$ is the Planck function at the observed frequency $(225 \mathrm{GHz})$ at temperature $T_{\mathrm{d}}$, and $\kappa_{v}$ is the absorption coefficient obtained from Table 1 in Ossenkopf \& Henning (1994; column for thin ice mantles and density of $10^{6} \mathrm{~cm}^{-3}$ ), interpolated for a frequency of $225 \mathrm{GHz}$, which provides a value of $\kappa_{v}=8.5 \times 10^{-3} \mathrm{~cm}^{2} \mathrm{~g}^{-1}$ taking into account the aforementioned gas-to-dust ratio.

We built the SED (Fig. 4) of all the ALMA detected sources to classify their evolutionary state, and then we adopted a dust temperature depending on class. For Class 0/I candidates, we used a temperature of $15 \pm 5 \mathrm{~K}$ based on the average temperature maps over the Class 0/I candidates in Lupus 1 and 3 (Fig. 2). These maps have been obtained from Herschel data (Teixeira et al., in prep.) following the methodology described 


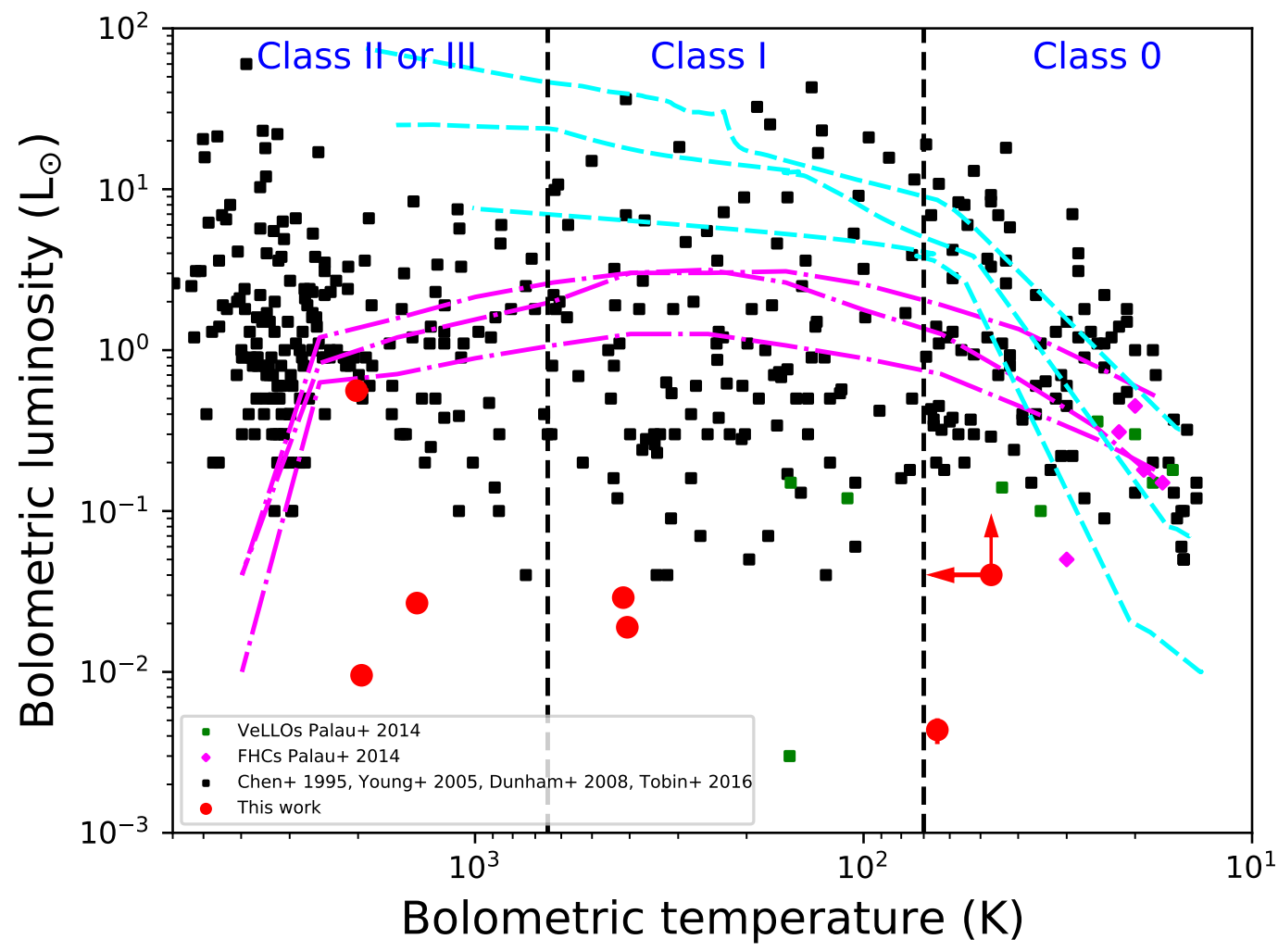

Fig. 5. Bolometric luminosity versus bolometric temperature. Black squares represent sources from Young \& Evans (2005) and Dunham et al. (2008) which show evidence of embedded low luminosity sources. Sources in Perseus from Tobin et al. (2016) are represented as open squares. Young Taurus (Chen et al. 1995) sources are displayed as black crosses. Green squares are known VeLLOs and the magenta diamonds are the First Hydrostatic Cores from Palau et al. (2014). The vertical dashed lines mark the Class 0-I and Class I-II bolometric temperature boundaries from Chen et al. (1995). The cyan short-dashed lines represent the evolutionary tracks for the three models with different masses considered by Young \& Evans (2005). The magenta, dotted, and dot-dashed lines show the evolutionary tracks for three models considered by Myers et al. (1998). Red points including uncertainty represent values obtained in this papers. Lower limits are included as red arrows shows for J153914.

in Lombardi et al. (2014). Most of the ALMA detections in Lupus 1 lie in regions with temperatures close to $16.5 \mathrm{~K}$, with only one source (J153702) showing a value close to $19 \mathrm{~K}$. In the case of the Lupus 3 sources, the reported temperatures vary between $\sim 13$ and $19 \mathrm{~K}$, depending on their location within the cloud. Other examples of similar temperatures for Class $0 / \mathrm{I}$ in the literature can be found in Stutz et al. $(2010 ; 17 \pm 1 \mathrm{~K})$ for lowmass stars, or for BDs (Barrado et al. 2018), using a temperature convention of $15 \mathrm{~K}$. For less-evolved (starless) sources (ALMA detections without optical/IR counterpart), we use a temperature of $9 \pm 1 \mathrm{~K}$ as an intermediate value between $\sim 8.5$ and 10 (André et al. 2012, and references therein) or 7-13 K from earlier work (Evans et al. 2001). For spectroscopically confirmed Class II BDs, we used a temperature of $20 \mathrm{~K}$ (Pascucci et al. 2016). The temperature for V1094 Sco came from the formula $T=25\left(L_{*} / L_{\odot}\right)^{0.25} \mathrm{~K}$ (Andrews et al. 2013) as it is in the solarmass regime (see Sect. 3.7.3).

Mass uncertainties are calculated using the computed distance error, the flux error, and the temperature error. Opacity uncertainty is not included although we are aware that it is a major source of uncertainty for mass estimates. Using the opacity formula from Ward-Thompson et al. (2010), the masses would be larger by $60 \%$.

The range of masses of the ALMA detections is between 0.18 and $124 M_{\text {Jup }}$. All the masses are in the planetary mass regime, except that of V1094 Sco, which is a well-known protostar with a protoplanetary disk surrounding it (Ansdell et al. 2016).

\subsection{AzTEC: Mass estimates}

Masses derived from the AzTEC data are estimated in the same fashion as described in Sect. 3.4. In Table 6, we show the mass estimates based on the ALMA continuum detections together with the mass estimates and the sizes of the AzTEC clumps, as well as an estimate of the missing flux in our ALMA observations. We also show the cloud membership for each object (11 in Lupus 3 and 8 in Lupus 1).

In order to calculate the missing flux in the ALMA observations we first calculated the expected flux of the AzTEC clumps at the frequency of the ALMA observations, assuming a dust emissivity index of $\beta=1.8$. The missing flux estimate for V1094 Sco is not included in the table given the complexity of the dust emissivity index for this source (van Terwisga et al. 2018). There is a large mass difference between the AzTEC clumps and the compact sources detected with ALMA, the latter providing much smaller mass values. The percentage of missing flux with ALMA ranges between $\sim 82 \%$ to more than $\sim 99 \%$. In Sect. 4.2 we discuss the flux discrepancy.

There are five ALMA sources (J160658, J160804, ParLup 3-4, J1609200, and J1609201) not associated with any AzTEC clump. With the exception of Par-Lup 3-4, all these sources were serendipitously discovered in the ALMA field of view (see Sect. 3.6), where we expected to find another BD. In the case of Lup 706, we assumed that most of the emission originates from V1094 Sco. The rest of the ALMA detections are associated with AzTEC spatially resolved clumps 
Table 5. Source classification using different methods.

\begin{tabular}{lccccc}
\hline \hline Source & Previous classification & Counterpart & $\alpha_{\text {IR }}$ slope & $T_{\text {bol }}$ & Final classification \\
\hline ALMA J153702.653-331924.92 & No & No & - & - & Pre-BD phase candidate \\
ALMA J153914.996-332907.62 & No & Yes & - & $>$ Class 0 & $\begin{array}{c}\text { Class I candidate } \\
\text { ALMA J154228.675-334230.18 }\end{array}$ \\
ALMA J154229.778-334241.86 & No & No & - & - & Pre-BD phase candidate \\
Class 0/I candidate \\
ALMA J154456.522-342532.99 & No & Yes & Class I & Class 0/I & pre-BD phase candidate \\
ALMA J154458.061-342528.51 & No & No & - & - & pre-BD phase candidate \\
ALMA J154506.515-344326.15 & No & No & - & - & Pre-BD phase candidate \\
ALMA J154634.169-343301.90 & No & No & - & - & Pre-BD phase candidate \\
ALMA J160658.604-390407.88 & No & No & - & - & Pre-BD phase candidate \\
ALMA J160804.168-390452.84 & No & No & - & - & Pre-BD phase candidate \\
160826.8-384101 & Class II & Yes & Class II & Class II & Class II \\
V*V1094 Sco & Class II & Yes & Class II & Class II & Class II \\
Lup706 & Class II & Yes & Class I/II & Class I & Class I/II \\
Par-lup 3-4 & Class I/II & Yes & Class I & Class I & Class I/II \\
SONYC-Lup 3-7 & Class II & Yes & Class I/II & Class II & Class II \\
ALMA J160920.089-384515.92 & No & No & - & - & Pre-BD phase candidate \\
ALMA J160920.171-384456.40 & No & No & - & - & Pre-BD phase candidate \\
ALMA J160932.167-390832.27 & No & No & - & - & Pre-BD phase candidate \\
ALMA J161030.273-383154.52 & No & No & - & - & Pre-BD phase candidate \\
\hline
\end{tabular}

Table 6. Derived properties from ALMA and AzTEC detections.

\begin{tabular}{lccccc}
\hline \hline Name & $\begin{array}{c}\text { Mass (ALMA) } \\
{\left[M_{\text {Jup }}\right]}\end{array}$ & $\begin{array}{c}\text { Mass (AzTEC) } \\
{\left[M_{\text {Jup }}\right]}\end{array}$ & $\begin{array}{c}\text { Size (AzTEC) } \\
{[\text { AU] }]}\end{array}$ & $\begin{array}{c}\text { Lupus } \\
\text { cloud }\end{array}$ & $\begin{array}{c}\text { Missing } \\
\text { flux }[\%]\end{array}$ \\
\hline ALMA J153702.653-331924.92 & $0.9 \pm 0.3$ & - & - & 1 & - \\
ALMA J153914.996-332907.62 & $0.63 \pm 0.11$ & $18 \pm 9$ & 3270 & 1 & 97 \\
ALMA J154228.675-334230.18 & $5.4 \pm 1.1$ & $41 \pm 10$ & $<2680$ & 1 & 82 \\
ALMA J154229.778-334241.86 & $3.5 \pm 1.7$ & $11 \pm 6$ & 3580 & 1 & 83 \\
ALMA J154456.522-342532.99 & $4.8 \pm 1.0$ & $31 \pm 8$ & 3800 & 1 & 87 \\
ALMA J154458.061-342528.51 & $2.7 \pm 0.6$ & $18 \pm 4$ & 3800 & 1 & 86 \\
ALMA J154506.515-344326.15 & $1.1 \pm 0.3$ & $51 \pm 12$ & 3380 & 1 & 99 \\
ALMA J154634.169-343301.90 & $1.4 \pm 0.3$ & $180 \pm 40$ & 7580 & 1 & 99 \\
ALMA J160658.604-390407.88 & $1.0 \pm 0.2$ & - & - & 3 & - \\
ALMA J160804.168-390452.84 & $1.7 \pm 0.4$ & - & - & 3 & - \\
160826.8-384101 & $1.1 \pm 0.4$ & - & - & 3 & - \\
V1094 Sco & $124 \pm 35$ & $82 \pm 10$ & 5030 & 3 & - \\
Lup706 & $0.20 \pm 0.12$ & - & - & 3 & - \\
Par-lup 3-4 & $0.19 \pm 0.08$ & - & - & 3 & - \\
SONYC-Lup 3-7 & $0.18 \pm 0.07$ & - & - & 3 & - \\
ALMA J160920.089-384515.92 & $1.8 \pm 0.4$ & - & - & 3 & - \\
ALMA J160920.171-384456.40 & $1.8 \pm 0.5$ & - & - & 3 & - \\
ALMA J160932.167-390832.27 & $1.2 \pm 0.4$ & $92 \pm 25$ & 5000 & 3 & 99 \\
ALMA J161030.273-383154.52 & $1.4 \pm 0.3$ & - & - & 3 & - \\
\hline
\end{tabular}

except for $\mathrm{J} 154228$, which is associated with a point-like source in the AzTEC maps (see Fig. B.4). SONYC-Lup 3-7 and J161030, detected with ALMA, were not covered by the AzTEC map. J153702 and 160826.8-384101 were noisy maps and discarded.

The masses of the AzTEC cores with no ALMA continuum detections are also given in Table 7.

\subsection{ALMA sources without optical/IR counterpart}

The following 12 sources detected in our ALMA survey have no optical or infrared counterpart: J153702, J154228, J154456,
J154458, J154506, J154634, J160658, J160804, J1609200, J1609201, J160932, and J161030. Six of these sources (J153702, $\mathrm{J} 160658$, J160804, J1609200, J1609201, and J161030) were serendipitously detected inside the ALMA primary beam at a distance greater than $3^{\prime \prime}$ from the nominal position of the spectroscopically confirmed Class II sources originally targeted. The remaining six sources (J154228, J154456, J154458, J154506, $\mathrm{J} 154634$, and J160932) are detected inside the ALMA primary beam of the observed pre-BD candidates from our AzTEC catalog.

J153702 is located at a distance of $6.9^{\prime \prime}$ from the Class II object 153703.1-331927 (ALMA phase centre). J160658 is 
Table 7. AzTEC: densities (critical and observed) and radii (critical and observed) at a temperature of $9 \mathrm{~K}$ for the ALMA non-detections.

\begin{tabular}{|c|c|c|c|c|c|c|}
\hline Name & $\begin{array}{l}\text { Mass } \\
{\left[M_{\odot}\right]}\end{array}$ & $\begin{array}{c}n_{\text {crit }} \\
{\left[\mathrm{cm}^{-3}\right]}\end{array}$ & $\begin{array}{c}n_{\mathrm{obs}} \\
{\left[\mathrm{cm}^{-3}\right]}\end{array}$ & $\begin{array}{l}R_{\max } \\
{[\mathrm{AU}]}\end{array}$ & $\begin{array}{l}R_{\mathrm{obs}} \\
{[\mathrm{AU}]}\end{array}$ & Dynamical state \\
\hline AzTEC-lup1-99 & 0.055 & $3.6 \times 10^{6}$ & $2.4 \times 10^{4}$ & 760 & 4000 & Stable \\
\hline AzTEC-lup1-103 & 0.07 & $2.2 \times 10^{6}$ & $1.7 \times 10^{4}$ & 960 & 4930 & Stable \\
\hline AzTEC-lup1-109 & 0.085 & $1.5 \times 10^{6}$ & $1.5 \times 10^{4}$ & 1170 & 5460 & Stable \\
\hline AzTEC-lup1-111 & 0.068 & $2.4 \times 10^{6}$ & $1.5 \times 10^{4}$ & 930 & 4980 & Stable \\
\hline AzTEC-lup1-57 & 0.085 & $1.5 \times 10^{6}$ & $1.9 \times 10^{4}$ & 1170 & 5060 & Stable \\
\hline AzTEC-lup1-67 & 0.11 & $9.2 \times 10^{5}$ & $1.4 \times 10^{4}$ & 1490 & 6100 & Stable \\
\hline AzTEC-lup1-114 & 0.10 & $1.1 \times 10^{6}$ & $1.1 \times 10^{4}$ & 1390 & 6450 & Stable \\
\hline AzTEC-lup1-84 & 0.048 & $4.7 \times 10^{6}$ & $3.2 \times 10^{4}$ & 660 & 3510 & Stable \\
\hline AzTEC-lup1-104 & 0.10 & $1.0 \times 10^{6}$ & $1.3 \times 10^{4}$ & 1430 & 6090 & Stable \\
\hline AzTEC-lup1-101 & 0.11 & $9.0 \times 10^{5}$ & $1.2 \times 10^{4}$ & 1510 & 6390 & Stable \\
\hline AzTEC-lup1-119 & 0.062 & $2.9 \times 10^{6}$ & $1.9 \times 10^{4}$ & 850 & 4510 & Stable \\
\hline AzTEC-lup1-124 & 0.11 & $9.7 \times 10^{5}$ & $1.1 \times 10^{4}$ & 1460 & 6580 & Stable \\
\hline AzTEC-lup1-52 & 0.15 & $5.2 \times 10^{5}$ & $1.4 \times 10^{4}$ & 1990 & 6580 & Stable \\
\hline AzTEC-lup1-54 & 0.28 & $1.4 \times 10^{5}$ & $9.6 \times 10^{3}$ & 3780 & 9320 & Stable \\
\hline AzTEC-lup1-94 & 0.12 & $7.6 \times 10^{5}$ & $1.1 \times 10^{4}$ & 1640 & 6680 & Stable \\
\hline AzTEC-lup1-123 & 0.048 & $4.7 \times 10^{6}$ & $2.3 \times 10^{4}$ & 660 & 3890 & Stable \\
\hline AzTEC-lup3-15 & 0.12 & $7.6 \times 10^{5}$ & $1.1 \times 10^{4}$ & 1640 & 6720 & Stable \\
\hline AzTEC-lup3-20 & 0.077 & $1.8 \times 10^{6}$ & $1.3 \times 10^{4}$ & 1060 & 5540 & Stable \\
\hline AzTEC-lup3-12 & 0.28 & $1.0 \times 10^{5}$ & $1.3 \times 10^{4}$ & 4260 & 8390 & Stable \\
\hline AzTEC-lup3-10 & 0.034 & $6.7 \times 10^{6}$ & $4.9 \times 10^{4}$ & 520 & 2710 & Stable \\
\hline AzTEC-lup3-5 & 1.3 & $4.6 \times 10^{3}$ & $3.2 \times 10^{4}$ & 20160 & 10480 & Unstable \\
\hline AzTEC-lup3-14 & 0.13 & $6.1 \times 10^{5}$ & $1.1 \times 10^{4}$ & 1830 & 6950 & Stable \\
\hline AzTEC-lup3-19 & 0.037 & $5.7 \times 10^{6}$ & $5.3 \times 10^{4}$ & 570 & 2710 & Stable \\
\hline AzTEC-lup3-8 & 1.0 & $7.7 \times 10^{3}$ & $4.3 \times 10^{4}$ & 15510 & 8710 & Unstable \\
\hline AzTEC-lup3-4 & 1.0 & $7.7 \times 10^{3}$ & $3.0 \times 10^{4}$ & 15460 & 9790 & Unstable \\
\hline AzTEC-lup3-13 & 0.54 & $2.8 \times 10^{4}$ & $1.7 \times 10^{4}$ & 8200 & 9680 & Stable \\
\hline AzTEC-lup3-9 & 0.22 & $1.7 \times 10^{5}$ & $2.0 \times 10^{4}$ & 3300 & 6790 & Stable \\
\hline AzTEC-lup3-16 & 0.079 & $1.8 \times 10^{6}$ & $1.5 \times 10^{4}$ & 1080 & 5350 & Stable \\
\hline
\end{tabular}

located at a distance of $3.1^{\prime \prime}$ from the Class II object $160658.7-390405$, that is located at $157 \pm 3 \mathrm{pc}$ according to the Gaia detection. J160804 is at a distance of 8.0" from the Class II source $160804.8-390449$, the source that was originally targeted in our survey. J161030 is at a distance of 5.0" from 161030.6-383151, the source originally targeted by ALMA. $\mathrm{J} 1609200$ and J1609201 are two sources detected in the same ALMA field of view (see Fig. B.1), located at 10.5" and 15.7" distance, respectively from the phase centre, which was the position of the Class II source 160920.8-384510. There is an optical/infrared counterpart close to J1609201 at 1.7", including Gaia measurements, but the high precision astrometry of Gaia as well as the accurate absolute astrometry for this source reveals that the counterpart are not associated with J1609201. The 2MASS counterpart in Gaia is 16092031-3844568 (Cutri et al. 2003).

The remaining sources are seen inside the ALMA primary beam centred at the AzTEC pre-BD candidates of our sample. J154228 is located 9.7" from the ALMA phase centre. The NASA/IPAC Extragalactic Database (NED) catalogue lists the closest object to the source as extragalactic and located at a distance of 3.7", which makes a physical association unlikely given the absolute position accuracy of ALMA. J154456 and J154458 fall inside the same primary beam. The separation between these two sources is $19.6^{\prime \prime}$, and they are located at $6.2^{\prime \prime}$ and $13.4^{\prime \prime}$, respectively, from the phase centre. The closest opti- cal object found is SSTSL2 J154456.77-342532.3 at 3.1" and $16.4^{\prime \prime}$ distance from J154456 and J154458, respectively. The ALMA detection of J154456 is spatially resolved with a Gaussian deconvolved size of $0.84^{\prime \prime} \pm 0.06^{\prime \prime} \times 0.28 \pm 0.08^{\prime \prime}$. J154506, $\mathrm{J} 160932$, and $\mathrm{J} 154634$ are located at distances of 12.0", 11.9", and $14.8^{\prime \prime}$, respectively, from their phase centres.

At the distance of Lupus, we found that the ALMA compact continuum emission mentioned above implies substellar masses below $10 M_{\text {Jup }}$, which are well inside the substellar regime independent of the values of temperature or opacity coefficient that we adopted. We checked for possible extragalactic contaminants in the NED and we found no clear detections associated with any of the detected objects. All these sources except J153702 and $\mathrm{J} 161030$, are located well within or quite close to the Lupus dust filaments as seen in the Herschel and AzTEC maps (see Figs. 1 and 2). Therefore, they are probably associated with the Lupus molecular clouds and we classify them as pre-BD candidates or deeply embedded proto-BD candidates. Future gas observations with better sensitivity at the positions of these candidates should help to confirm or reject their Galactic nature and the membership of each source to the complex.

\subsection{ALMA sources with optical/infrared counterpart}

The sources described in this subsection have optical or infrared counterparts, or a combination of both. Using the classification 
tools described above (counterpart presence, $\alpha_{\mathrm{IR}}$ slope, and $T_{\mathrm{bol}}$ ), we make an attempt to classify their state of evolution, considering the uncertainty in their physical association to the Lupus clouds, and the incomplete SEDs for most of them. We identify one new Class 0/I proto-BD candidate, one Class I proto-BD candidate (we note that these sources may instead be pre-BDs if their optical counterpart are not in fact associated with them), and confirm five Class II substellar objects previously known.

\subsubsection{Class $0 / 1$}

J154229 (Fig. 3) is detected and spatially resolved in this work for the first time. We report a $26 \sigma$ detection and a deconvolved size of $0.77^{\prime \prime} \times 0.64^{\prime \prime}$, with a mass of $3.5 \pm 1.7 M_{\text {Jup }}$. We found a counterpart at several wavelengths at a distance between $0.1^{\prime \prime}$ and 0.4" (WFI, WISE, Spitzer/IRAC, Spitzer/MIPS). The counterpart in other wavebands are positionally consistent with a single source. The $\mathrm{J} 154229 \alpha_{\mathrm{IR}}$ slope is compatible with a Class I source, but the bolometric temperature indicates that it is most likely a Class 0 object that is very close to the boundary with Class I. Therefore we classify it as a proto-BD candidate in a transition stage between Class 0 to Class I (see Table 4). The SED of J154229 is similar to that of the Taurus proto-BD Class 0/I candidate J041757-B found by Barrado et al. (2009). The pre$\mathrm{BD}$ candidate $\mathrm{J} 154228$ is located inside the same primary beam at a distance of $17.9^{\prime \prime}$.

\subsubsection{Class I}

J153914 has not been previously reported in the literature. We detect an ALMA continuum point source corresponding to a mass of $0.63 M_{\text {Jup }}$. This source has an optical counterpart $0.7^{\prime \prime}$ distant from the ALMA detection. The source is detected at three different optical bands showing a steep positive slope. There are no infrared counterpart associated with this source, which complicates the confirmation of the physical association to the WFI source with the ALMA source. If confirmed, this source would be a Class I proto-BD candidate or a background object. On the contrary, if there is not such an association then it would be classified as a pre-BD or deeply embedded proto-BD candidate. Future infrared observations are needed to clarify the exact nature of this source.

\subsubsection{Class $\mathrm{I} / \mathrm{II}$ and Class II}

160826.8-384101 is a spectroscopically confirmed Class II source in Lupus 3 (Comerón et al. 2009a). The central object has $T_{\text {eff }}=2900 \mathrm{~K}$ and a mass of $0.06 M_{\odot}$ (Comerón et al. 2009a). ALMA continuum emission at $1.3 \mathrm{~mm}$ is detected at $26 \sigma$ (Fig. B.2), and it is not spatially resolved. The disk mass is $1.1 \pm 0.4 M_{\text {Jup }}$ at a distance of $165 \pm 4 \mathrm{pc}$. The bolometric temperature is $\sim 1411 \mathrm{~K}$, in the Class II range.

V1094 Sco is a Class II YSO in the stellar mass regime (Frasca et al. 2017) discovered by Krautter et al. (1997). Our high signal-to-noise ratio image spatially resolves the dust disk. We estimated a disk mass of $124 \pm 35 M_{\text {Jup }}$ and the disk temperature is $22.5 \mathrm{~K}$, at a distance of $154.7 \pm 1.1 \mathrm{pc}$. The object is extensively discussed in several papers (Baraffe et al. 2015; Alcalá et al. 2017; Frasca et al. 2017). The central source has $T_{\text {eff }}=4205 \pm 193 \mathrm{~K}$ with a mass of $1.10 M_{\odot}$ and it is classified as K6 (Frasca et al. 2017). The SED is very complete including detections from the optical to the infrared at different separations (WFI, DENIS, 2MASS, WISE, Spitzer-MIPS, SpitzerIRAC, Spitzer-MIPS, SPIRE, PACS, LABOCA, and Akari).
Lup 706 is a spectroscopically confirmed Class II substellar object, previously discovered and classified as a BD (López Martí et al. 2005). We report a non-spatially resolved detection (Fig. B.2). The mass of the disk is $0.20 \pm 0.12 M_{\text {Jup }}$ at a distance of $191 \pm 29 \mathrm{pc}$. The nominal distance is on the high side but the large error bar of the distance does not rule out cloud membership. The projected separation between Lup 706 and V1094 Sco is $16^{\prime \prime}$.

The central object has $T_{\text {eff }}=2750 \mathrm{~K}$ and a mass of $0.06_{-0.02}^{+0.03} M_{\odot}$ and is classified as an M7.5 BD (Alcalá et al. 2014; Mužić et al. 2014). Lup 706 has been classified as a Class II object (Alcalá et al. 2014, 2017), the same classification that we obtained from the SED. Although the bolometric temperature is $\sim 415 \mathrm{~K}$, below the Class II source limit, it is possible that, as we see in Par-Lup 3-4, the inclination of the surrounding disk is responsible for this low temperature estimate. Both sources, Par-Lup 3-4 and Lup 706 were classified as subluminous by Alcalá et al. (2014). Future SED modeling could give us more information about the inclination angle. A recent study (Sanchis et al. 2020) reports a lower dust disk mass of Lup 706 from ALMA Band 7 data, but their results are consistent with ours within $1 \sigma$ using the absorption coefficient in Ossenkopf \& Henning (1994).

Par-Lup 3-4 (Fig. B.2) was discovered by Nakajima et al. (2000) and confirmed spectroscopically as a Lupus 3 member by Comerón et al. (2003). It is a VLM star with a mass of $0.13 M_{\odot}$, M4.5 spectral type and $T_{\text {eff }}=3089 \pm 246 \mathrm{~K}$ (Alcalá et al. 2017; Manara et al. 2013; Frasca et al. 2017). We report a spatially unresolved detection. The mass of the disk is $0.19 \pm 0.08 M_{\text {Jup }}$ at a distance of $155 \pm 14 \mathrm{pc}$. We also detect $\mathrm{CO}(2-1)$ gas emission associated with this source at velocities close to the $V_{\mathrm{LSR}}$ of Lupus, which confirms its association to the molecular cloud. Par-Lup 3-4 is classified as a Class II source in the literature, with an almost edge-on disk (Huélamo et al. 2010). Interestingly, additional ALMA data on this source in Band 7 have revealed the presence of a bipolar molecular outflow (Santamaría-Miranda et al. 2020). The bolometric temperature is $\sim 405 \mathrm{~K}$, in the Class I regime, but the source is under-luminous (Comerón et al. 2003).

SONYC-Lup 3-7 is a spectroscopically confirmed Class II substellar source in Lupus 3 discussed in Alcalá et al. (2014) and Mužić et al. (2014). Our ALMA data (Fig. B.2) show an unresolved object with a disk mass of $0.18 \pm 0.07 M_{\text {Jup }}$ at a distance of $151 \pm 6 \mathrm{pc}$. The bolometric temperature obtained from the SED is 1959 K. Alcalá et al. (2014) and Mužić et al. (2014) found that the central object has a temperature of 2600-2850 K and a mass of $0.03 \pm 0.01 M_{\odot}$. We confirm the source as a Class II, based on its SED. A recent study (Sanchis et al. 2020) reported a dust disk mass of SONYC-Lup 3-7 in Band 7 and that is compatible with our results within $2 \sigma$.

\section{Discussion}

\subsection{Spatial distribution: Detections versus non-detections}

The spatial distribution of the Lupus 1 objects detected with AzTEC is shown in Fig. 1. The AzTEC data reveal two filaments, the primary extending more than $1.5^{\circ}$ in the Northern part of the map (left panel Fig. 1), and a second smaller filament located southwest from the primary. Almost all the sources are in the primary filament, although there are several non-detections in the secondary filament. There are three AzTEC cores outside the two filaments. Of these three cases outside of the filaments, only one (J153702) is detected with ALMA, and this source was 
serendipitously detected when we pointed to a spectroscopically confirmed Class II BD. For Lupus 3, the distribution is slightly different (right panel in Fig. 1). There is only one filament that has several sources along it, including ALMA detections without an optical/IR counterpart, such as J160804 or 160932. However, there are also some ALMA detections located at the outskirts of the filament (J1609200, J1609201 and J161030). Finally, there are nine AzTEC detections are located at a considerable distance from the centre of the filament, that are distributed across the whole map. For comparison, we checked that some proto-BD candidates in other regions are also detected outside the main filaments. For example, J041757 is at the outskirts of the B213 main filament of Taurus (Palau et al. 2012). We conclude that there is no specific preferred cloud environment where AzTEC sources detected with ALMA tend to cluster.

\subsection{Detection rate of pre- and proto-BD candidates and large-scale core properties}

The ALMA detection rate of the whole sample was $23 \%$ (15 of the 64 pointings), or $18 \%$ ( 5 of 40 ) if we reduce the sample to the initial classification of 33 pre-stellar and 7 Class 0 and I objects we surveyed. A similar work in Barnard 30 (Huélamo et al. 2017) using LABOCA and ALMA obtained a detection rate of $17 \%$. Both ALMA experiments were designed to detect faint sources even in the worst-case scenario where all the emission was extended in an area equivalent to the ALMA largest angular scale, which in our case was $\sim 11^{\prime \prime}$. The selection strategy (see Sect. 2.1) was built using an AzTEC beam size of $\sim 30^{\prime \prime}$ and cores with peak intensities between 30 and $100 \mathrm{mJy}$. We adopted a very conservative approach whereby the emission from the dusty envelope or disk surrounding a very young brown dwarf could be as large as the ALMA largest angular size (LAS) of $11^{\prime \prime}$ for our observations, that is, $\sim 1650 \mathrm{AU}$ at $\sim 150 \mathrm{pc}$, and a uniform distribution over that envelope. Considering the ALMA synthesized beam of our observations $\left(\sim 0.85^{\prime \prime}\right.$ or $\left.\sim 130 \mathrm{AU}\right)$ and the fact that our data are sensitive enough to detect sources at the $5 \sigma$ level based on the average AzTEC rms, this means that nondetections are probably related to the source size and not to the sensitivity, further suggesting the AzTEC clump material is distributed mainly on large scales. This implies that we are filtering most of the extended emission in our ALMA observations.

\subsection{Evolution of the ALMA starless cores: Final mass of the pre-BD candidates}

For the ALMA pre-BD candidates, we estimated the final masses of the central compact object by assuming a core formation efficiency in the substellar regime of $30 \%$ (and a temperature of $9 \mathrm{~K}$ ), which is similar to that inferred for low-mass cores (Motte et al. 1998). Therefore, for the pre-BD candidates detected with ALMA, we added $30 \%$ of the AzTEC clump mass to the ALMA mass. As a result we find that all of these pre-BD candidates have the capacity to evolve to form substellar objects with final masses between 9 and $60 M_{\text {Jup }}$ (see Fig. 6). We conclude that the six ALMA compact sources detected inside the six AzTEC clumps, and without counterpart at shorter wavelengths, may end up as substellar objects.

\subsection{Nature of ALMA detections without optical/infrared counterparts}

In this section, we discuss the nature of the ALMA detections without counterparts based on the assumption that they are star-

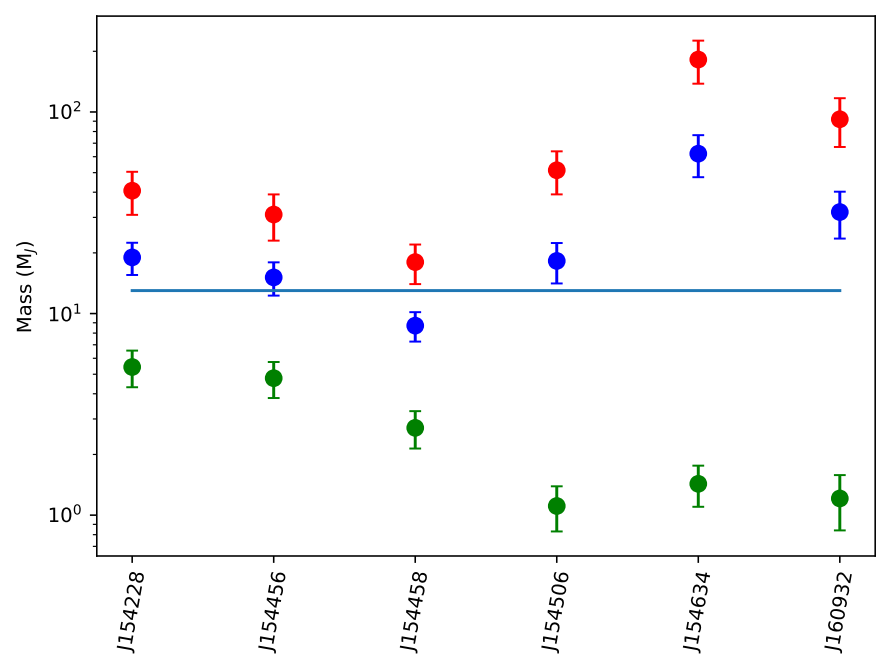

Fig. 6. Mass of the ALMA pre-BDs candidates without an opti$\mathrm{cal} /$ infrared counterpart at a temperature of $9 \mathrm{~K}$. Red points are the AzTEC masses. Green points are the ALMA masses. Blue points are the assumed final masses as a combination of the ALMA mass and the AzTEC mass assuming a core SFE of $30 \%$. Horizontal line marks the deuterium-burning limit.

less cores (pre-BD). We note, however, that we cannot exclude the possibility that they are embedded proto-BDs without any counterparts detected in the infrared images.

\subsubsection{Infall according to turbulent fragmentation}

The theory of turbulent fragmentation (Padoan \& Nordlund 2004) is based on collapse due to externally driven supersonic turbulence. The critical mass for the collapse of a Bonnor-Ebert sphere (Bonnor 1956) is defined as:

$M_{\mathrm{BE}}\left[M_{\odot}\right]=3.3\left(\frac{T}{10[\mathrm{~K}]}\right)^{3 / 2}\left(\frac{n_{\text {crit }}}{10^{3}\left[\mathrm{~cm}^{-3}\right]}\right)^{-1 / 2}$,

where $T$ is the temperature in $\mathrm{K}$ and $n_{\text {crit }}$ is the critical density in $\mathrm{cm}^{-3}$. For the following calculations, we assume the BonnorEbert mass is the one from AzTEC cores or ALMA compact detections. The uncertainty in the Bonnor-Ebert sphere mass is estimated using the mass uncertainty in Table 6 and the temperature error with a fixed value of $1 \mathrm{~K}$, as done in Sect. 3.4. The critical radius $\left(R_{\text {crit }}\right)$ can be simplified to

$R_{\text {crit }}[\mathrm{AU}]=\left(\frac{2.83 \times 10^{16} M\left[M_{\odot}\right]}{n_{\text {crit }}\left[\mathrm{cm}^{-3}\right]}\right)^{1 / 3}$,

where $M$ is the mass of the source.

The observed density $\left(n_{\mathrm{obs}}\right)$ is defined as the ratio of the hydrogen column density to the linear size of the source, and it is calculated using the mass of the source estimated from the observations and the size of the emitting region. For those ALMA sources that are not spatially resolved we use the synthesized beam as an upper limit to their size.

According to this theory, cores with an observed density that is lower than the critical value $\left(n_{\text {obs }}<n_{\text {crit }}\right)$ are expected to be transient cores. On the contrary, if the density of the core is greater than the critical density $\left(n_{\text {obs }}>n_{\text {crit }}\right)$, the core should be gravitationally unstable and is expected to be in the pre-stellar phase. A comparison of the average radius of a source $\left(R_{\mathrm{obs}}\right)$ to the critical radius $\left(R_{\text {crit }}\right)$ corresponding to the critical density 
Table 8. ALMA: densities (critical and observed) and radii (critical and observed) at a temperature of $9 \mathrm{~K}$.

\begin{tabular}{lccccc}
\hline \hline Name & $\begin{array}{c}n_{\text {crit }} \\
{\left[\mathrm{cm}^{-3}\right] \pm \%}\end{array}$ & $\begin{array}{c}n_{\text {obs }} \\
{\left[\mathrm{cm}^{-3}\right] \pm \%}\end{array}$ & $\begin{array}{c}R_{\max } \\
{[\mathrm{AU}]}\end{array}$ & $\begin{array}{c}R_{\text {obs }} \\
{[\mathrm{AU}]}\end{array}$ & Dynamical state $^{(1)}$ \\
\hline $\mathrm{J} 153702$ & $1.2 \times 10^{10} \pm 66$ & $>6.3 \times 10^{7}$ & $13 \pm 3$ & $<72$ & Stable? \\
$\mathrm{J} 154228$ & $3.0 \times 10^{8} \pm 53$ & $>1.2 \times 10^{9}$ & $79 \pm 15$ & $<49$ & Unstable \\
$\mathrm{J} 154456$ & $3.8 \times 10^{8} \pm 52$ & $1.7 \times 10^{9} \pm 78$ & $70 \pm 13$ & 43 & Unstable \\
$\mathrm{J} 154458$ & $1.2 \times 10^{9} \pm 54$ & $>2.5 \times 10^{8}$ & $40 \pm 8$ & $<67$ & Stable? \\
$\mathrm{J} 154506$ & $7.1 \times 10^{9} \pm 61$ & $>9.6 \times 10^{7}$ & $16 \pm 4$ & $<68$ & Stable? \\
$\mathrm{J} 154634$ & $1.0 \times 10^{10} \pm 62$ & $>7.8 \times 10^{7}$ & $13 \pm 3$ & $<68$ & Stable? \\
$\mathrm{J} 160658$ & $4.1 \times 10^{9} \pm 59$ & $>1.2 \times 10^{8}$ & $21 \pm 4$ & $<69$ & Stable? \\
$\mathrm{J} 160804$ & $3.0 \times 10^{9} \pm 60$ & $>1.4 \times 10^{8}$ & $25 \pm 5$ & $<69$ & Stable? \\
$\mathrm{J} 1609200$ & $2.8 \times 10^{9} \pm 59$ & $>1.1 \times 10^{8}$ & $26 \pm 5$ & $<75$ & Stable? \\
$\mathrm{J} 1609201$ & $2.8 \times 10^{9} \pm 68$ & $>1.5 \times 10^{8}$ & $26 \pm 6$ & $<69$ & Stable? \\
$\mathrm{J} 160932$ & $1.1 \times 10^{10} \pm 80$ & $>7.4 \times 10^{7}$ & $13 \pm 4$ & $<69$ & Stable? \\
$\mathrm{J} 161030$ & $4.7 \times 10^{9} \pm 59$ & $>1.1 \times 10^{8}$ & $20 \pm 4$ & $<69$ & Stable? \\
\hline
\end{tabular}

Notes. ${ }^{(1)}$ Stable vs. infalling described in Sect. 4.4, the question mark indicates upper limits.

Table 9. ALMA: densities (critical and observed) and radii (critical and observed) at a temperature of $15 \mathrm{~K}$.

\begin{tabular}{lccccc}
\hline \hline Name & $\begin{array}{c}n_{\text {crit }} \\
{\left[\mathrm{cm}^{-3}\right] \pm \%}\end{array}$ & $\begin{array}{c}n_{\text {obs }} \\
{\left[\mathrm{cm}^{-3}\right] \pm \%}\end{array}$ & $\begin{array}{c}R_{\max } \\
{[\mathrm{AU}]}\end{array}$ & $\begin{array}{c}R_{\text {obs }} \\
{[\mathrm{AU}]}\end{array}$ & Dynamical state \\
\hline $\mathrm{J} 153702$ & $2.6 \times 10^{11} \pm 50$ & $>2.9 \times 10^{7}$ & $3 \pm 1$ & $<72$ & Stable? \\
$\mathrm{J} 154228$ & $6.6 \times 10^{9} \pm 32$ & $>5.5 \times 10^{8}$ & $22 \pm 2$ & $<49$ & Stable? \\
$\mathrm{J} 154456$ & $8.6 \times 10^{9} \pm 30$ & $7.6 \times 10^{8} \pm 69$ & $19 \pm 2$ & 43 & Stable? \\
$\mathrm{J} 154458$ & $2.7 \times 10^{10} \pm 33$ & $>1.1 \times 10^{8}$ & $11 \pm 1$ & $<67$ & Stable? \\
$\mathrm{J} 154506$ & $1.6 \times 10^{11} \pm 43$ & $>4.3 \times 10^{7}$ & $4 \pm 1$ & $<68$ & Stable? \\
$\mathrm{J} 154634$ & $2.3 \times 10^{11} \pm 45$ & $>3.5 \times 10^{7}$ & $4 \pm 1$ & $<68$ & Stable? \\
$\mathrm{J} 160658$ & $9.2 \times 10^{10} \pm 40$ & $>5.5 \times 10^{7}$ & $6 \pm 1$ & $<69$ & Stable? \\
$\mathrm{J} 160804$ & $6.8 \times 10^{10} \pm 42$ & $>6.4 \times 10^{7}$ & $7 \pm 1$ & $<69$ & Stable? \\
$\mathrm{J} 1609200$ & $6.4 \times 10^{10} \pm 41$ & $>5.1 \times 10^{7}$ & $7 \pm 1$ & $<75$ & Stable? \\
$\mathrm{J} 1609201$ & $6.2 \times 10^{10} \pm 53$ & $>6.6 \times 10^{7}$ & $7 \pm 1$ & $<69$ & Stable? \\
$\mathrm{J} 160932$ & $2.4 \times 10^{11} \pm 68$ & $>3.4 \times 10^{7}$ & $4 \pm 1$ & $<69$ & Stable? \\
$\mathrm{J} 161030$ & $1.1 \times 10^{11} \pm 41$ & $>5.1 \times 10^{7}$ & $5 \pm 1$ & $<69$ & Stable? \\
\hline
\end{tabular}

is also used to infer the energetic state of sources. Sources that are spatially unresolved, with $R_{\text {obs }}$ smaller than $R_{\text {crit }}$ indicate that the core is unstable. For resolved sources, $R_{\text {crit }}$ sets a boundary between stable cores $\left(R_{\mathrm{obs}}>R_{\text {crit }}\right)$ and unstable $\left(R_{\mathrm{obs}}<R_{\text {crit }}\right)$ cores.

We calculate the critical density and the observed density for the ALMA pre-BD candidates using two values of temperature: $T=9 \mathrm{~K}$, which is more characteristic of pre-BD cores (Table 8); and $T=15 \mathrm{~K}$ for deeply embedded protostars (Table 9), as we did in Sect. 3.4. We also computed these parameters for the AzTEC cores with ALMA detections (see Tables 10 and 11), and for the AzTEC clumps without ALMA detections Table 7.

An unresolved ALMA sources is presumed to be collapsing if its radius is smaller than the value given in Table 8 . For the resolved source $\mathrm{J} 154456$, we obtained density and size values that suggests it is collapsing.

For the spatially resolved larger AzTEC cores, we find observed densities that are one to two orders of magnitude smaller than the critical densities, regardless of the adopted temperature $(9$ or $15 \mathrm{~K})$. This remains true even if we include the $1 \sigma$ uncertainties. Their radii are also larger than the $R_{\text {crit }}$. All this suggests they are transient cores. The unresolved AzTEC core hosting $\mathrm{J} 154228$ could be unstable if its radius is smaller than $\sim 530 \mathrm{AU}$.

We carried out the calculation of the energetic states of the rest of the AzTEC cores where no ALMA detections were obtained and found that all the cores in the substellar regime seem to be stable.

In Fig. 7, we compare the derived $n_{\text {crit }}$ and $n_{\text {obs }}$ for the ALMA sources, assuming a temperature of $9 \mathrm{~K}$. As seen, two of our pre-BD candidates are in the infalling regime. The rest of the cores are not resolved with ALMA and therefore we cannot exclude that they also may be unstable. We compared our results with those from Huélamo et al. (2017) by computing the source masses in their work using a temperature of $9 \mathrm{~K}$ instead of the $15 \mathrm{~K}$ assumed in the original paper. With a temperature of $15 \mathrm{~K}$ and the uncertainties related to the mass (of a factor of 4 ) it is not entirely clear whether the cores in Barnard 30 are in collapse. However, using a temperature of $9 \mathrm{~K}$, the pre-BD core candidates in Barnard 30 clearly lie in the unstable regime. On the other hand, our Cycle 3 observations were more sensitive than the Cycle 1 observations in Huélamo et al. (2017), which might explain our ability to make detections in the apparently non-collapsing regime. 
Table 10. AzTEC: densities (critical and observed) and radii (critical and observed) at a temperature of $9 \mathrm{~K}$.

\begin{tabular}{lccccl}
\hline \hline Name & $\begin{array}{c}n_{\text {crit }} \\
{\left[\mathrm{cm}^{-3}\right] \pm \%}\end{array}$ & $\begin{array}{c}n_{\text {obs }} \\
{\left[\mathrm{cm}^{-3}\right] \pm \%}\end{array}$ & $\begin{array}{c}R_{\max } \\
{[\mathrm{AU}]}\end{array}$ & $\begin{array}{c}R_{\text {obs }} \\
{[\mathrm{AU}]}\end{array}$ & Dynamical state \\
\hline $\mathrm{J} 154228$ & $5.3 \times 10^{6} \pm 59$ & $>5.7 \times 10^{4}$ & $590 \pm 130$ & $<2680$ & Stable \\
$\mathrm{J} 154456$ & $9.0 \times 10^{6} \pm 59$ & $1.5 \times 10^{4} \pm 30$ & $450 \pm 100$ & 3800 & Stable \\
$\mathrm{J} 154558$ & $2.6 \times 10^{7} \pm 59$ & $8.9 \times 10^{3} \pm 30$ & $270 \pm 60$ & 3800 & Stable \\
$\mathrm{J} 154506$ & $3.3 \times 10^{6} \pm 59$ & $3.6 \times 10^{4} \pm 30$ & $750 \pm 160$ & 3380 & Stable \\
$\mathrm{J} 154634$ & $2.6 \times 10^{5} \pm 59$ & $1.1 \times 10^{4} \pm 30$ & $2650 \pm 560$ & 7580 & Stable \\
$\mathrm{J} 160932$ & $9.3 \times 10^{5} \pm 62$ & $2.1 \times 10^{4} \pm 39$ & $1410 \pm 320$ & 5000 & Stable \\
\hline
\end{tabular}

Table 11. AzTEC: densities (critical and observed) and radii (critical and observed) at a temperature of $15 \mathrm{~K}$.

\begin{tabular}{lccccc}
\hline \hline Name & $\begin{array}{c}n_{\text {crit }} \\
{\left[\mathrm{cm}^{-3}\right] \pm \%}\end{array}$ & $\begin{array}{c}n_{\text {obs }} \\
{\left[\mathrm{cm}^{-3}\right] \pm \%}\end{array}$ & $\begin{array}{c}R_{\max } \\
{[\mathrm{AU}]}\end{array}$ & $\begin{array}{c}R_{\text {obs }} \\
{[\mathrm{AU}]}\end{array}$ & Dynamical state \\
\hline $\mathrm{J} 154228$ & $1.4 \times 10^{8} \pm 37$ & $>2.4 \times 10^{4}$ & $150 \pm 20$ & $<2680$ & Stable? \\
$\mathrm{J} 154456$ & $2.3 \times 10^{8} \pm 37$ & $6.5 \times 10^{3} \pm 22$ & $120 \pm 15$ & 3800 & Stable \\
$\mathrm{J} 154558$ & $6.8 \times 10^{8} \pm 37$ & $3.8 \times 10^{3} \pm 22$ & $70 \pm 10$ & 3800 & Stable \\
$\mathrm{J} 154506$ & $8.5 \times 10^{7} \pm 37$ & $1.5 \times 10^{4} \pm 22$ & $190 \pm 30$ & 3380 & Stable \\
$\mathrm{J} 154634$ & $6.8 \times 10^{6} \pm 37$ & $4.8 \times 10^{3} \pm 22$ & $680 \pm 90$ & 7580 & Stable \\
$\mathrm{J} 160932$ & $2.4 \times 10^{7} \pm 43$ & $8.9 \times 10^{3} \pm 32$ & $360 \pm 60$ & 5000 & Stable \\
\hline
\end{tabular}

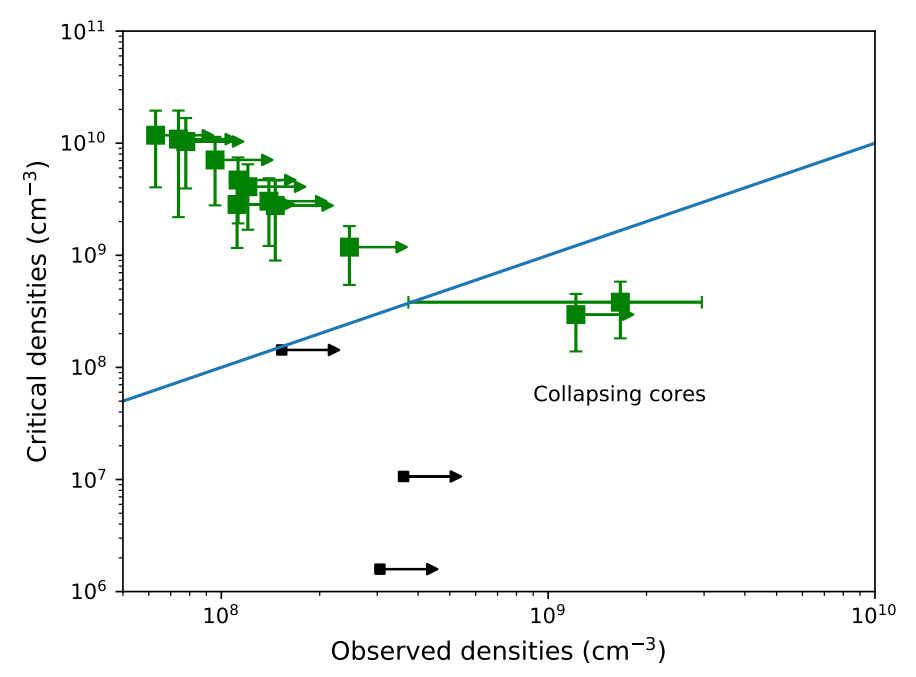

Fig. 7. Critical densities vs observed densities at an adopted temperature $T=9 \mathrm{~K}$ using ALMA. Green symbols shows the sources studied in this work and the square is J154456. Black symbols shows the sources in Huélamo et al. (2017). Lower limits are included as arrows. Below the blue line the cores should be unstable, and therefore collapsing.

\subsection{2. $r^{-2}$ density profile}

The analysis in the previous section shows that most of the AzTEC pre-BD cores with ALMA detections are stable. However, this result is in conflict with the fact that we already detect very compact sources at these core centers, which indicates ongoing collapse. Huélamo et al. (2017) proposed that this kind of configuration in pre-BD objects (stable largescale cores with a compact source inside) could be the "tip of the iceberg" of a larger-scale collapse. Thus, ALMA compact sources would have been formed as the product of gravitational contraction, where an $r^{-2}$ density profile is naturally developed with a finite infall velocity in a gravitationally unstable background (Naranjo-Romero et al. 2015; Gómez et al. 2007; Mohammadpour \& Stahler 2013; Larson 1969). This is naturally expected in the scenario of global hierarchical collapse (Vázquez-Semadeni et al. 2019).

Using the $r^{-2}$ density profile and the flux detected with AzTEC we estimate the expected mass inside the average ALMA beam having a FWHM of $0.91^{\prime \prime}$ ( 139 AU). Results can be seen in Table 12, where we give the ratio between the estimated and the observed masses. There are three sources that lie on the 1:1 relation. Hence, it seems that this set of pre-BD candidates could be the product of gravitational collapse. Three of the sources are not close to a ratio of 1 and two of them are the same sources that seem to be unstable when applying the turbulent fragmentation theory. The difference between the sources with a ratio close to 1 and the other three might be related to their evolutionary stage: ratios between 0.14 and 0.07 would suggest density profiles steeper than $r^{-2}$ and this would indicate that they have already accreted more mass onto the central object, which is consistent with their being more evolved. The other three sources show a Bonnor-Ebert isothermal profile, therefore indicating that they are less evolved.

The assumed $r^{-2}$ density profile predicts sizes smaller than the ALMA synthesized beam, with the exception of J154456 (the only pre-BD spatially resolved in the sample). This is in agreement with the unavailability of resolving spatially most of these compact structures with this interferometer configuration (see Table 13).

In Fig. 8, we compare the mass derived from the ALMA observations (at $T=9 \mathrm{~K}$ ) to the mass estimated from an $r^{-2}$ density profile. The estimated mass error is based on the ALMA and AzTEC mass errors. Barnard 30 pre-BD candidates are also included. We note that Huélamo et al. (2017) considered typical uncertainties of a factor of 4 in the mass estimate. The results in Barnard 30 are very similar to those we found in our Lupus sample, and they also seem to be in a state of gravitational contraction. Thus, both studies ultimately reach a very similar 
Table 12. Estimated masses of the ALMA pre-BD core candidates within an ALMA beam, assuming a $r^{-2}$ density profile from AzTEC.

\begin{tabular}{lcccccc}
\hline \hline \multirow{2}{*}{ Name } & \multicolumn{2}{c}{ AzTEC $(9 \mathrm{~K})$} & & \multicolumn{2}{c}{ ALMA mass $(9 \mathrm{~K})$} & \\
\cline { 2 - 3 } & $\begin{array}{c}\text { Mass } \\
{\left[M_{\text {Jup }}\right]}\end{array}$ & $\begin{array}{c}\text { Radii } \\
{[\text { AU }]}\end{array}$ & & $\begin{array}{c}\text { Estimated } \\
{\left[M_{\text {Jup }}\right]}\end{array}$ & $\begin{array}{c}\text { Observed } \\
{\left[M_{\text {Jup }}\right]}\end{array}$ & Ratio $^{(1)}$ \\
\hline ALMA J154228.675-334230.18 & $41 \pm 10$ & $<2680$ & & $0.75 \pm 0.18$ & $5.4 \pm 1.1$ & 0.14 \\
ALMA J154456.522-342532.99 & $31 \pm 8$ & 3800 & & $0.35 \pm 0.09$ & $4.8 \pm 1.0$ & 0.07 \\
ALMA J154458.061-342528.51 & $18 \pm 4$ & 3800 & & $0.20 \pm 0.05$ & $2.7 \pm 0.6$ & 0.07 \\
ALMA J154506.515-344326.15 & $51 \pm 12$ & 3380 & & $1.0 \pm 0.3$ & $1.1 \pm 0.3$ & 0.94 \\
ALMA J154634.169-343301.90 & $180 \pm 40$ & 7580 & & $1.6 \pm 0.4$ & $1.4 \pm 0.3$ & 1.15 \\
ALMA J160932.167-390832.27 & $100 \pm 30$ & 5000 & & $1.5 \pm 0.40$ & $1.2 \pm 0.4$ & 1.21 \\
\hline
\end{tabular}

Notes. ${ }^{(1)}$ Estimated ALMA mass/observed ALMA mass.

Table 13. Predicted pre-BD cores radii containing the mass measured with ALMA and assuming a $r^{-2}$ density profile, versus the observed radii with ALMA.

\begin{tabular}{lcc}
\hline \hline Name & $\begin{array}{c}\text { Estimated } \\
{[\mathrm{AU}]}\end{array}$ & $\begin{array}{c}\text { Observed } \\
{[\mathrm{AU}]}\end{array}$ \\
\hline ALMA J154228.675-334230.18 & 48 & $<49$ \\
ALMA J154456.522-342532.99 & 42 & 43 \\
ALMA J154458.061-342528.51 & 24 & $<67$ \\
ALMA J154506.515-344326.15 & 10 & $<68$ \\
ALMA J154634.169-343301.90 & 12 & $<69$ \\
ALMA J160932.167-390832.27 & 11 & $<69$ \\
\hline
\end{tabular}

conclusion: most of the objects detected with ALMA seem to be at the beginning of the large-scale contraction expected in a scaled-down version of a low-mass star formation scenario.

\subsection{The nature of J154229.778-334241.86}

J154229 is the only proto-BD candidate in our study whose SED is well populated. In the NED catalogue, there is a nearby infrared source located $0.24^{\prime \prime}$ distant, whose extragalactic nature has not been confirmed. To rule out the possibility that J154229 is extragalactic, meaning that it is, in fact, an active galactic nucleus (AGN), we compared its SED (see right panel in Fig. 9) with an average SED of radio-loud and radio-quiet AGN with different extinction values, following Palau et al. (2012). The differences, especially in the optical and in the millimetre, suggest it is not very likely that $\mathrm{J} 154229$ is an AGN. We compared the SED of J154229 with two other proto-BD candidates, J041757 and J042118 in Taurus (Barrado et al. 2009; Palau et al. 2012) (see left panel in Fig. 9). The similar shape of all three SEDs suggests J154229 is very likely a bona fide proto-BD candidate. One of these sources, J041757, has a cold dust envelope with a size of 1000 au and a mass of $5 M_{\text {Jup }}$, while the envelope of J154229 is three times larger ( 3500 AU) and more massive $\left(11 M_{\text {Jup }}\right)$. This difference may suggest that J15299 is slightly younger than J042118. We conclude that due to the similarities in the SED, J154229 is a promising proto-BD candidate. However, gas detection is necessary to confirm its association with the Lupus molecular cloud.

\subsection{Class II brown dwarfs: disk masses}

The properties of the disks of more evolved BDs can also shed light on the formation of substellar objects and on the possi-

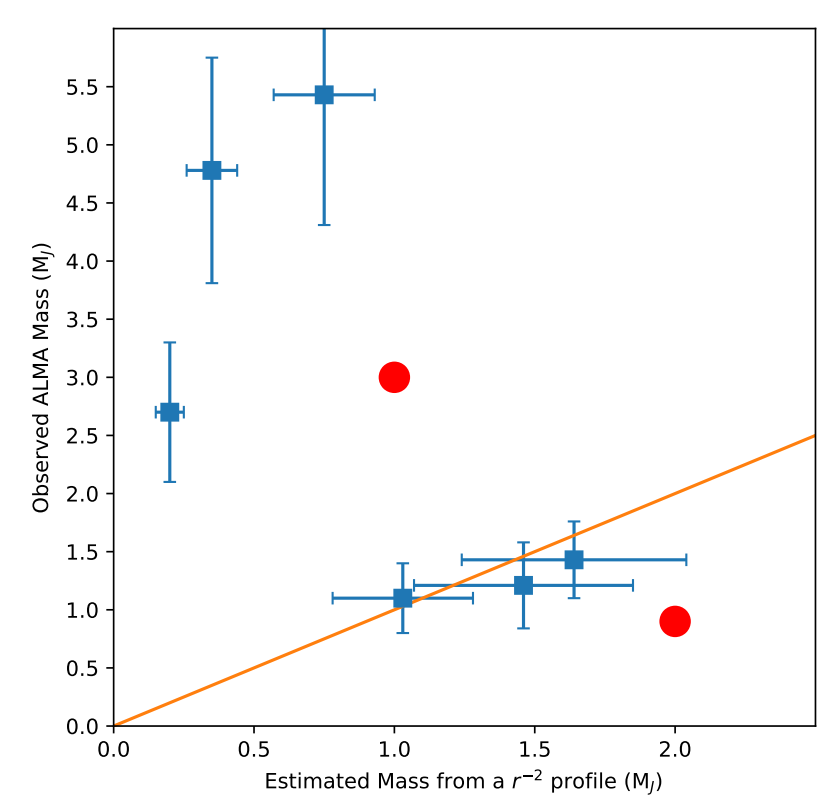

Fig. 8. Observed mass for the pre-BD vs. the estimated mass using a $r^{-2}$ density profile. Using that profile and the flux detected with AzTEC we estimate the expected mass inside the ALMA beam. Blue squares are sources in this work detected with ALMA. Red points are sources from Huélamo et al. (2017). The ratio between the observed and the estimated masses is close to $\sim 1$ (orange line) for half of the sources (see Sect. 4.4.2). The other three sources with steeper density profiles might be more evolved as they have accreted more mass onto the central object.

bility of planet formation in these sources. Theoretical predictions (Stamatellos \& Herczeg 2015) indicate that for central sources with equal masses, the disk masses are higher in sources formed by disk fragmentation compared to sources formed as a scaled-down version of low-mass stars. In order to compare different formation scenarios, we estimated the dust disk masses of the Class II sources in our sample along with previous Class II BDs observed with ALMA in several SFRs.

In the case of the Lupus sources, the mass of the central object was obtained from the literature (Comeron et al. 2009b; Alcalá et al. 2014) and adjusted to the new Gaia DR2 parallaxes. To do this, we rescaled the luminosity values from the literature using the new Gaia distances, then we interpolated the stellar mass from the position in the Hertzsprung-Russel diagram using the evolutionary models of Baraffe et al. (2015). We calculated the dust disk mass as described in Sect. 3.4 using the distances given in Table 3 and a value of $\kappa_{v}=8.5 \times 10^{-1} \mathrm{~cm}^{2} \mathrm{~g}^{-1}$ 

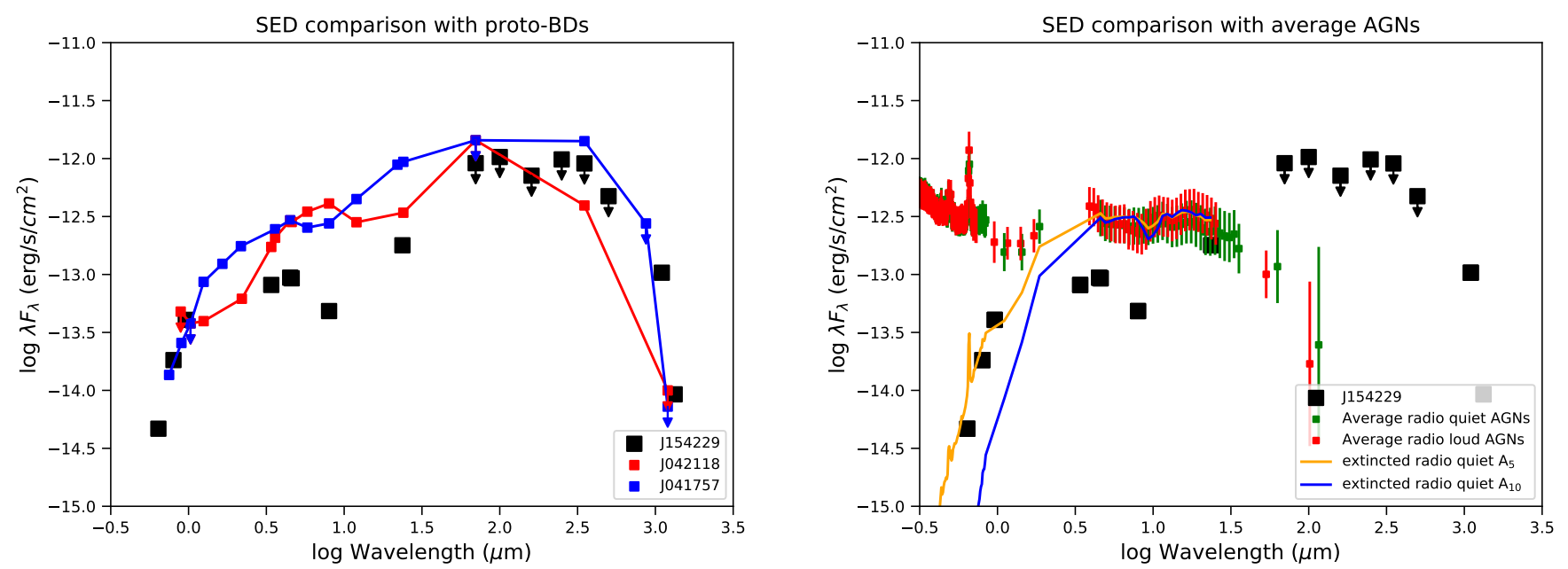

Fig. 9. Left panel: SED of J154229.778-334241.86 (black) compared with proto-BD candidates J041757 (blue) and J042118 (red) including upper limits (marked with arrows). This figure is based on Fig. 8 from Palau et al. (2012). Right panel: SED of J154229.778-334241.86 (black) compared with average SEDs for radio-quiet AGN (green) with a $V$-band extinction of 5 (orange) and $V$-band extinction of 10 (blue), and radio-loud AGN (red) from Shang et al. (2011). This figure is based on Fig. 9 from Palau et al. (2012).

Table 14. Disk dust masses for the spectroscopically confirmed Class II BDs our sample. Temperature is constant at $20 \mathrm{~K}$.

\begin{tabular}{lc}
\hline \hline Name & $\begin{array}{c}\text { Disk dust mass } \\
{\left[M_{\oplus}\right]}\end{array}$ \\
\hline 160826 & $3.4 \pm 0.2$ \\
Lup 706 & $0.6 \pm 0.2$ \\
Par-Lup 3-4 & $0.6 \pm 0.2$ \\
SONYC-Lup 3-7 & $0.58 \pm 0.09$ \\
\hline
\end{tabular}

(Ossenkopf \& Henning 1994). The range of masses we obtained is between 0.58 to $3.4 M_{\oplus}$ (see Table 14). We calculated upper limits for the Class II sources in Lupus 1 and 3 that were not detected with ALMA using the rms value in Table A.1. We included objects whose distances are compatible with the average distance derived for Lupus 1 and 3 (see Sect. 2.4) as well as BDs in our sample that are probably not Lupus members.

Having inferred the disk masses of our Class II substellar sources associated with Lupus, we compared them with BD and VLM star disks previously observed with ALMA. We searched for available Class II substellar sources with published ALMA detections and then we recalculated the masses using the fluxes provided in the literature and the above values of temperature and opacity (Ossenkopf \& Henning 1994). Using stellar parameters from the literature, the theoretical evolutionary models from Baraffe et al. (2015), and the new Gaia DR2 parallaxes, we calculated the masses for each BD and VLM star.

We included sources in several SFRs such as Ophiuchus (Testi et al. 2016), Upper Scorpius (van der Plas et al. 2016), Chamaeleon I (Pascucci et al. 2016), Taurus (Ricci et al. 2014; Ward-Duong et al. 2018), and Lupus (Ansdell et al. 2016; Sanchis et al. 2020). We used the stellar parameters in Alves de Oliveira et al. (2012), Mužić et al. (2012), Manara et al. (2015) to obtain the stellar masses for the Ophiuchus sources. From Upper Scorpius (van der Plas et al. 2016) we chose sources with spectral type later than M 4. The stellar parameters from Scholz et al. (2007), van der Plas et al. (2016).

For Taurus we collected sources from two different works: Ward-Duong et al. (2018) and Ricci et al. (2014). From the latter we included three BDs; however the error in the flux is not provided so we assumed the rms error. We used the stellar parameters from Ricci et al. (2013) and Andrews et al. (2013). From Ward-Duong et al. (2018), we selected sources with a spectral type later than M 4 choosing the flux obtained with the natural weighting algorithm. Stellar properties are indicated in the same work. From Chamaeleon I (Pascucci et al. 2016) we selected sources with spectral types later than M4, and stellar parameters from Manara et al. (2014, 2016). Finally, we incorporated other sources in Lupus with spectral type later than M4 that are not part of our survey (Ansdell et al. 2016; Sanchis et al. 2020); stellar masses for these sources are in Alcalá et al. (2014, 2017), Mužić et al. (2014). The stellar and disk masses for all these regions, together with those of Lupus 1 and 3, are shown in Fig. 10.

Regarding the formation mechanism, we included the predictions from Stamatellos \& Herczeg (2015) about disk fragmentation and ejection in Fig. 10. About $40 \%$ of all the detected substellar sources in the plot are in the expected range, although there are several sources whose values are even higher than expected for disk fragmentation. The blue and green areas represent the expected scaling relations between disk mass and stellar mass extrapolated from the stellar regime using three different models (D'Antona \& Mazzitelli 1997; Baraffe et al. 1998; Siess et al. 2000) and all the BDs, including the upper limits are in agreement with these relations. The two formation methods overlap in the BD regime and part of the detected objects with ALMA are consistent with both formation scenarios. However, the upper limits already indicate that these sources seem inconsistent with the disk fragmentation scenario. On the other hand, upper limits may be formed by the turbulent fragmentation and the whole dataset of detected BD with ALMA seem to favor the star-like scenario as a dominant mechanism.

The largest dust disk mass detected among the objects in our Lupus sample is $3.4 M_{\oplus}$. The minimum disk mass expected for planet formation is around $10 M_{\oplus}$ (Payne \& Lodato 2007; Testi et al. 2016). Thus, planet formation in the substellar regime in these objects seems not to be possible unless planets, planetesimals or planetary cores) are already formed in these systems. Recent results from studies of protoplanetary disks (Andrews et al. 2018) suggest that planets are formed much earlier than 


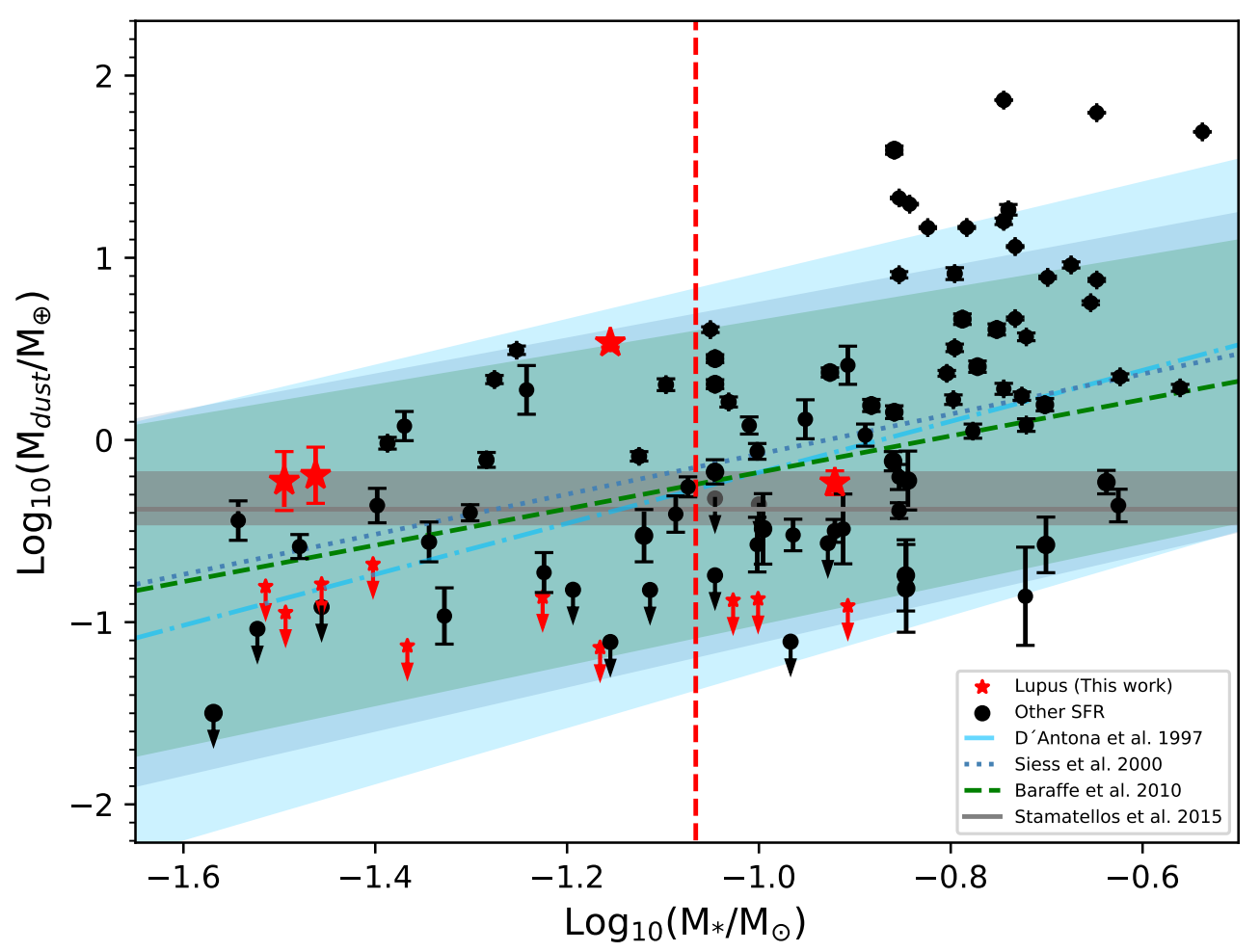

Fig. 10. Dust disk masses as a function of the central object mass $\left(M_{*}\right)$ for Class II BDs. Red stars mark sources in this paper including upper limits (marked with arrows). Black circles correspond to BD and VLM stars in other SFRs such as Ophiuchus (Testi et al. 2016), Upper Scorpius (van der Plas et al. 2016), Chamaeleon I (Pascucci et al. 2016), Taurus (Ricci et al. 2014; Ward-Duong et al. 2018), and Lupus sources that are not part of our survey (Ansdell et al. 2016; Sanchis et al. 2020). Upper limits are included as arrows. All the objects were observed with ALMA and masses were recalculated using $T=20 \mathrm{~K}$ for consistency. The grey area represents the predictions for disk fragmentation in Stamatellos \& Herczeg (2015), while the blue and green areas are the scaling relations between disk mass and stellar mass for protoplanetary disks derived by Andrews et al. (2013) using three different sets of model calculations (D’Antona \& Mazzitelli 1997; Baraffe et al. 1998; Siess et al. 2000). The red vertical line represents the limit between BDs and stars.

previously thought. This can explain the low dust disk masses that we measured if we assume that planet formation in BDs follows the core accretion or the disk instability mechanism in the stellar regime.

Finally, a word of caution should be included. The relation between disk temperature and luminosity is still under debate (van der Plas et al. 2016; Pascucci et al. 2016; Ward-Duong et al. 2018) given that the models make various assumptions that might not be correct. The luminosity, temperature, and disk radii are intrinsically related. As it was seen for some sources in Ophiuchus (Testi et al. 2016), the radii can be smaller than expected. This will affect the disk temperature. We chose a fixed temperature of $20 \mathrm{~K}$ for Class II BDs without any uncertainty. If we use $T=25\left(L / L_{\odot}\right)^{0.25}$ instead, the disk masses are $\sim 3$ times larger.

Future observations with better angular resolution, deeper observations to detect fainter sources, and future modeling are required to gain a fuller understanding of the dominant BD formation mechanism and to decide whether planet formation is possible in these disks, based on the detection similar substructures as those seen in the stellar regime.

\subsection{Exploring the dominant scenario for $B D$ formation}

In the previous sections, we present a review of the characteristics of pre-BD core candidates and Class II brown dwarfs according to various formation scenarios.

In conclusion, $40 \%$ of the detected Class II BDs in all the SFRs, are consistent with a disk fragmentation scenario, according to the theoretical predictions (Stamatellos \& Whitworth
2009). However, the entire sample follows the scaling relation between the stellar mass and the disk mass obtained in the stellar regime. Our detection rate is similar to the previous work of Huélamo et al. (2017). Given that our sample was bigger it is natural that we find a larger total number of candidates. We expect that observations with either increased sensitivity or larger LAS will increase the number of detected sources and help to finally confirm their nature (see Sect. 4.2). We think that photoevaporation is not the dominant formation mechanism in BDs for the two Lupus regions and Barnard 30 because they share similar very young populations, but reside in different large scale environments - in fact, there are no massive stars in Lupus 1.

As a final remark, we note that although we checked each source in the NED to check their classification as possible extragalactic sources, we cannot fully ensure their Galactic nature except for those sources that were detected by Gaia and spectroscopically confirmed as YSOs. The sources are mainly located in the cloud filament and are probably associated with the cloud, but only future gas detections at the velocity of the cloud would confirm the membership of each source to the complex.

\section{Conclusions}

In this work, we present a search of substellar objects at the earliest stages using high sensitivity ALMA observations in the Lupus 1 and 3 star-forming regions. Our target selection is based on sources found in AzTEC $(1.1 \mathrm{~mm})$ maps and complemented with Class II BDs from the literature. We supplement 
our observations with optical/IR archival data. The main objective of this work is to test and constrain BD formation theories by identifying pre- and proto-BDs and classifying them according to their evolutionary state. Our main results as follows.

- We detected sources in 15 of the 64 ALMA pointings. The total number of compact sources detected is 19 . We report masses between 0.33 and $124 M_{\mathrm{Jup}}$. The ALMA detection rate was $\sim 23 \%$. The ALMA non-detections may be related to the source size, with spatial scales larger than the ALMA LAS dominating the emission.

- The number of newly classified candidate objects is 14 . We classified 12 sources as pre-BDs or deeply embedded protostar candidates because they do not have optical/infrared counterpart. We classified one Class 0/I and one Class I candidate based on their SED slope and the $T_{\text {bol }}$.

- We detected continuum emission in five more Class II sources that were previously selected from the literature and we measured their disk masses, which span values between 0.58 and $3.4 M_{\oplus}$

- We studied the nature of the 12 sources that we classified as pre-BD or deeply embedded protostar candidates. For these sources, we compared the estimated density with the critical density from a Bonnor-Ebert isothermal sphere, concluding that one of the sources seems to be collapsing. Second, we explored an alternative path comparing the estimated ALMA masses with the masses that should be obtained from an $r^{-2}$ density profile, and we concluded that the pre-BDs core candidates could be the result of a large-scale gravitational collapse.

- We estimated the final mass of the pre-BD candidates, assuming a core efficiency of $30 \%$ using the mass derived from AzTEC and adding the ALMA mass, and we find that all the sources could end up as substellar objects.

- The Class 0/I candidate J154229 is at the boundary between Class 0 and I, based on bolometric temperature, and thanks to the efficient sampling of its SED, it has proven to be a promising proto-BD candidate. The SED suggests this object has already formed a BD in its core. Its SED is similar to that of other known proto-BDs and different from the typical SEDs of AGN.

- We compared the dust disk masses for the objects that we classified as Class II with previous studies of YSOs in other SFRs and obtained similar results. A scaled-down version of low-mass star formation may be the dominant scenario, however, it is fair to note that the disk fragmentation may be responsible for a non-negligible number of BDs.

Future observations are needed to confirm the nature of the ALMA pre- and proto-BD candidates. Optical/infrared spectroscopic studies for J153914 and J154229 are needed to confirm them as proto-BDs. In addition, the detection of gas associated with any of the candidates in the sample would confirm their membership to the Lupus molecular complex.

Acknowledgements. This paper makes use of the following ALMA data: ADS/JAO.ALMA\#2015.1.00512.S. ALMA is a partnership of ESO (representing its member states), NSF (USA) and NINS (Japan), together with NRC (Canada), MOST and ASIAA (Taiwan), and KASI (Republic of Korea), in cooperation with the Republic of Chile. The Joint ALMA Observatory is operated by ESO, AUI/NRAO and NAOJ. A.S.-M., I.G.-M., M.R.S. and A.B. acknowledge support from the "Iniciativa Científica Milenio" via Núcleo Milenio de Formación Planetaria. A.B. acknowledges support from FONDECYT Regular grant N. 1190748. IdG-M is partially supported by MCIU-AEI (Spain) grant AYA2017-84390-C2-R (co-funded by FEDER). NH acknowledges financial support from the Spanish State Research Agency (AEI) Project No. ESP201787676-C5-1-R and from project No. MDM-2017-0737 Unidad de Excelencia "María de Maeztu" - Centro de Astrobiología (INTA-CSIC). The National Radio Astronomy Observatory is a facility of the National Science Foundation operated under cooperative agreement by Associated Universities, Inc. KM acknowledges funding by the Science and Technology Foundation of Portugal (FCT), grants No. IF/00194/2015 and PTDC/FIS-AST/28731/2017. A.P. acknowledges financial support from CONACyT and UNAM-PAPIIT IN113119 grant, México.

\section{References}

Adams, F. C., Lada, C. J., \& Shu, F. H. 1987, ApJ, 312, 788

Alcalá, J. M., Natta, A., Manara, C. F., et al. 2014, A\&A, 561, A2 Alcalá, J. M., Manara, C. F., Natta, A., et al. 2017, A\&A, 600, A20 Alves de Oliveira, C., Moraux, E., Bouvier, J., \& Bouy, H. 2012, A\&A, 539, A151

Alves de Oliveira, C., Moraux, E., Bouvier, J., et al. 2013, A\&A, 549, A123 André, P., Ward-Thompson, D., \& Greaves, J. 2012, Science, 337, 69 Andrews, S. M., Rosenfeld, K. A., Kraus, A. L., \& Wilner, D. J. 2013, ApJ, 771, 129

Andrews, S. M., Huang, J., Pérez, L. M., et al. 2018, ApJ, 869, L41

Ansdell, M., Williams, J. P., van der Marel, N., et al. 2016, ApJ, 828, 46 Ansdell, M., Williams, J. P., Trapman, L., et al. 2018, ApJ, 859, 21 Apai, D., Tóth, L. V., Henning, T., et al. 2005, A\&A, 433, L33 Baade, D., Meisenheimer, K., Iwert, O., et al. 1999, The Messenger, 95, 15 Bailer-Jones, C. A. L. 2015, PASP, 127, 994

Baraffe, I., Chabrier, G., Allard, F., \& Hauschildt, P. H. 1998, A\&A, 337, 403 Baraffe, I., Homeier, D., Allard, F., \& Chabrier, G. 2015, A\&A, 577, A42 Barnard, E. 1927, Catalogue of 349 Dark Objects in the Sky (Chicago: Univ. of Chicago Press)

Barrado, D., Morales-Calderón, M., Palau, A., et al. 2009, A\&A, 508, 859 Barrado, D., de Gregorio Monsalvo, I., Huélamo, N., et al. 2018, A\&A, 612, A79 Barrado y Navascués, D., Stauffer, J. R., Bouvier, J., Jayawardhana, R., \& Cuillandre, J. C. 2004, ApJ, 610, 1064

Basri, G., Marcy, G. W., \& Graham, J. R. 1995, in Amer. Astron. Soc. Meet. Abstr., \#186, BAAS, 27, 1214

Bate, M. R. 2014, MNRAS, 442, 285

Bate, M. R., Bonnell, I. A., \& Bromm, V. 2002, MNRAS, 332, L65 Bayo, A., Barrado, D., Stauffer, J., et al. 2011, A\&A, 536, A63 Bohlin, R. C., Savage, B. D., \& Drake, J. F. 1978, ApJ, 224, 132 Bonnor, W. B. 1956, MNRAS, 116, 351

Bourke, T. L., Myers, P. C., Evans, N. J., II, et al. 2006, ApJ, 649, L37

Bouy, H., Huélamo, N., Martín, E. L., et al. 2007, A\&A, 463, 641

Bowler, B. P., Liu, M. C., Kraus, A. L., Mann, A. W., \& Ireland, M. J. 2011, ApJ, 743, 148

Bowler, B. P., Liu, M. C., Kraus, A. L., \& Mann, A. W. 2014, ApJ, 784, 65

Caballero, J. A., Béjar, V. J. S., Rebolo, R., et al. 2007, A\&A, 470, 903

Cambrésy, L. 1999, A\&A, 345, 965

Chabrier, G., Johansen, A., Janson, M., \& Rafikov, R. 2014, in Protostars and Planets VI, eds. H. Beuther, R. S. Klessen, C. P. Dullemond, \& T. Henning, 619

Chen, H., Myers, P. C., Ladd, E. F., \& Wood, D. O. S. 1995, ApJ, 445, 377

Comerón, F. 2008, in The Lupus Clouds, ed. B. Reipurth, 295

Comerón, F., Fernández, M., Baraffe, I., Neuhäuser, R., \& Kaas, A. A. 2003, A\&A, 406, 1001

Comerón, F., Spezzi, L., \& López Martí, B. 2009a, A\&A, 500, 1045

Comeron, F., Spezzi, L., \& Lopez Marti, B. 2009b, VizieR Online Data Catalog: $\mathrm{J} / \mathrm{A}+\mathrm{A} / 500 / 1045$

Cutri, R. M., Skrutskie, M. F., van Dyk, S., et al. 2003, VizieR Online Data Catalog: II/246

Dang-Duc, C., Phan-Bao, N., \& Dao-Van, D. T. 2016, A\&A, 588, L2

D’Antona, F., \& Mazzitelli, I. 1997, Mem. Soc. Astron. It., 68, 807

de Gregorio-Monsalvo, I., Barrado, D., Bouy, H., et al. 2016, A\&A, 590, A79

Dunham, M. M., Crapsi, A., Evans, N. J., II, et al. 2008, ApJS, 179, 249

Enoch, M. L., Evans, N. J., II, Sargent, A. I., \& Glenn, J. 2009, ApJ, 692, 973

Epchtein, N., de Batz, B., Copet, E., et al. 1994, Ap\&SS, 217, 3

Evans, N. J., II, Rawlings, J. M. C., Shirley, Y. L., \& Mundy, L. G. 2001, ApJ, 557,193

Fadda, D., Jannuzi, B. T., Ford, A., \& Storrie-Lombardi, L. J. 2004, AJ, 128, 1

Frasca, A., Biazzo, K., Alcalá, J. M., et al. 2017, A\&A, 602, A33

Gaia Collaboration (Brown, A. G. A., et al.) 2018, A\&A, 616, A1

Galli, P. A. B., Joncour, I., \& Moraux, E. 2018, MNRAS, 477, L50

Galli, P. A. B., Loinard, L., Bouy, H., et al. 2019, A\&A, 630, A137

Gómez, G. C., Vázquez-Semadeni, E., Shadmehri, M., \& Ballesteros-Paredes, J. 2007, ApJ, 669, 1042

Greene, T. P., Wilking, B. A., Andre, P., Young, E. T., \& Lada, C. J. 1994, ApJ, 434,614

Griffin, M. J., Abergel, A., Abreu, A., et al. 2010, A\&A, 518, L3

Hales, A. S., Pérez, S., Saito, M., et al. 2018, ApJ, 859, 111

Hester, J. J., Scowen, P. A., Sankrit, R., et al. 1996, AJ, 111, 2349

Hildebrand, R. H. 1983, QJRAS, 24, 267 
Hoyle, F. 1953, ApJ, 118, 513

Huélamo, N., Bouy, H., Pinte, C., et al. 2010, A\&A, 523, A42

Huélamo, N., de Gregorio-Monsalvo, I., Palau, A., et al. 2017, A\&A, 597, A17

Jayawardhana, R., Mohanty, S., \& Basri, G. 2003, ApJ, 592, 282

Kauffmann, J., Bertoldi, F., Bourke, T. L., et al. 2011, MNRAS, 416, 2341

Kawabe, R., Hara, C., Nakamura, F., et al. 2018, ApJ, 866, 141

Kim, G., Lee, C. W., Maheswar, G., et al. 2019, ApJS, 240, 18

Klein, R., Apai, D., Pascucci, I., Henning, T., \& Waters, L. B. F. M. 2003, ApJ, 593, L57

Krautter, J., Wichmann, R., Schmitt, J. H. M. M., et al. 1997, A\&AS, 123, 329

Larson, R. B. 1969, MNRAS, 145, 271

Lee, C. W., Bourke, T. L., Myers, P. C., et al. 2009, ApJ, 693, 1290

Lee, C. W., Kim, M.-R., Kim, G., et al. 2013, ApJ, 777, 50

Lee, C. W., Kim, G., Myers, P. C., et al. 2018, ApJ, 865, 131

Lodieu, N., Caux, E., Monin, J. L., \& Klotz, A. 2002, A\&A, 383, L15

Lodieu, N., Zapatero Osorio, M. R., Béjar, V. J. S., \& Peña Ramírez, K. 2018, MNRAS, 473, 2020

Lombardi, M., Bouy, H., Alves, J., \& Lada, C. J. 2014, A\&A, 566, A45

López Martí, B., Eislöffel, J., \& Mundt, R. 2005, A\&A, 440, 139

Luhman, K. L., Adame, L., D’Alessio, P., et al. 2007, ApJ, 666, 1219

Luhman, K. L., Herrmann, K. A., Mamajek, E. E., Esplin, T. L., \& Pecaut, M. J. 2018, AJ, 156, 76

Machida, M. N., Tomisaka, K., Matsumoto, T., \& Inutsuka, S.-I. 2008, ApJ, 677, 327

Machida, M. N., Inutsuka, S.-I., \& Matsumoto, T. 2009, ApJ, 699, L157

Manara, C. F., Testi, L., Rigliaco, E., et al. 2013, A\&A, 551, A107

Manara, C. F., Testi, L., Natta, A., et al. 2014, A\&A, 568, A18

Manara, C. F., Testi, L., Natta, A., \& Alcalá, J. M. 2015, A\&A, 579, A66

Manara, C. F., Fedele, D., Herczeg, G. J., \& Teixeira, P. S. 2016, A\&A, 585, A136

Manara, C. F., Mordasini, C., Testi, L., et al. 2019, A\&A, 631, L2

McMullin, J. P., Waters, B., Schiebel, D., Young, W., \& Golap, K. 2007, in CASA Architecture and Applications, eds. R. A. Shaw, F. Hill, \& D. J. Bell, ASP Conf. Ser., 376, 127

Merín, B., Jørgensen, J., Spezzi, L., et al. 2008, ApJS, 177, 551

Mohammadpour, M., \& Stahler, S. W. 2013, MNRAS, 433, 3389

Morata, O., Palau, A., González, R. F., et al. 2015, ApJ, 807, 55

Motte, F., Andre, P., \& Neri, R. 1998, A\&A, 336, 150

Murakami, H., Baba, H., Barthel, P., et al. 2007, PASJ, 59, S369

Mužić, K., Scholz, A., Geers, V., Jayawardhana, R., \& Tamura, M. 2012, ApJ, 744,134

Mužić, K., Scholz, A., Geers, V. C., Jayawardhana, R., \& López Martí, B. 2014, ApJ, 785, 159

Mužić, K., Scholz, A., Geers, V. C., \& Jayawardhana, R. 2015, ApJ, 810, 159

Mužić, K., Scholz, A., Peña Ramírez, K., et al. 2019, ApJ, 881, 79

Myers, P. C., \& Ladd, E. F. 1993, ApJ, 413, L47

Myers, P. C., Adams, F. C., Chen, H., \& Schaff, E. 1998, ApJ, 492, 703

Nakajima, Y., Tamura, M., Oasa, Y., \& Nakajima, T. 2000, AJ, 119, 873

Naranjo-Romero, R., Vázquez-Semadeni, E., \& Loughnane, R. M. 2015, ApJ, 814,48

Natta, A., \& Testi, L. 2001, A\&A, 376, L22

Natta, A., Testi, L., Comerón, F., et al. 2002, A\&A, 393, 597

Natta, A., Testi, L., Muzerolle, J., et al. 2004, A\&A, 424, 603

Natta, A., Testi, L., \& Randich, S. 2006, A\&A, 452, 245

Olivares, J., Sarro, L. M., Bouy, H., et al. 2020, A\&A, 644, A7

Oppenheimer, B. R., Kulkarni, S. R., Matthews, K., \& Nakajima, T. 1995, Science, 270,1478

Ossenkopf, V., \& Henning, T. 1994, A\&A, 291, 943

Padoan, P., \& Nordlund, A. 2004, ApJ, 617, 559
Palau, A., de Gregorio-Monsalvo, I., Morata, Ò., et al. 2012, MNRAS, 424, 2778

Palau, A., Zapata, L. A., Rodríguez, L. F., et al. 2014, MNRAS, 444, 833

Pascucci, I., Testi, L., Herczeg, G. J., et al. 2016, ApJ, 831, 125

Payne, M. J., \& Lodato, G. 2007, MNRAS, 381, 1597

Peña Ramírez, K., Zapatero Osorio, M. R., \& Béjar, V. J. S. 2015, A\&A, 574, A118

Phan-Bao, N., Riaz, B., Lee, C.-F., et al. 2008, ApJ, 689, L141

Pilbratt, G. L., Riedinger, J. R., Passvogel, T., et al. 2010, A\&A, 518, L1

Poglitsch, A., Waelkens, C., Geis, N., et al. 2010, A\&A, 518, L2

Rebolo, R., Zapatero Osorio, M. R., \& Martín, E. L. 1995, Nature, 377, 129

Reipurth, B., \& Clarke, C. 2001, AJ, 122, 432

Riaz, B., Thompson, M., Whelan, E. T., \& Lodieu, N. 2015, MNRAS, 446, 2550

Riaz, B., Vorobyov, E., Harsono, D., et al. 2016, ApJ, 831, 189

Riaz, B., Briceño, C., Whelan, E. T., \& Heathcote, S. 2017, ApJ, 844, 47

Riaz, B., Thi, W. F., \& Caselli, P. 2018, MNRAS, 481, 4662

Riaz, B., Machida, M. N., \& Stamatellos, D. 2019, MNRAS, 486, 4114

Ricci, L., Isella, A., Carpenter, J. M., \& Testi, L. 2013, ApJ, 764, L27

Ricci, L., Testi, L., Natta, A., et al. 2014, ApJ, 791, 20

Ruíz-Rodríguez, D., Cieza, L. A., Williams, J. P., et al. 2018, MNRAS, 478, 3674

Saito, M., de Gregorio, I., \& Team SOLA 2015, in The Soul of Lupus with

ALMA (SOLA) Project Overview, eds. D. Iono, K. Tatematsu, A. Wootten, \& L. Testi, ASP Conf. Ser., 499, 215

Sanchis, E., Testi, L., Natta, A., et al. 2020, A\&A, 633, A114

Santamaría-Miranda, A., Cáceres, C., Schreiber, M. R., et al. 2018, MNRAS, 475, 2994

Santamaría-Miranda, A., de Gregorio-Monsalvo, I., Huélamo, N., et al. 2020, A\&A, 640, A13

Scholz, A., Jayawardhana, R., \& Wood, K. 2006, ApJ, 645, 1498

Scholz, A., Jayawardhana, R., Wood, K., et al. 2007, ApJ, 660, 1517

Scholz, A., Jayawardhana, R., Muzic, K., et al. 2012, ApJ, 756, 24

Shang, Z., Brotherton, M. S., Wills, B. J., et al. 2011, ApJS, 196, 2

Siess, L., Dufour, E., \& Forestini, M. 2000, A\&A, 358, 593

Siringo, G., Kreysa, E., Kovács, A., et al. 2009, A\&A, 497, 945

Skrutskie, M. F., Cutri, R. M., Stiening, R., et al. 2006, AJ, 131, 1163

Stamatellos, D., \& Herczeg, G. J. 2015, MNRAS, 449, 3432

Stamatellos, D., \& Whitworth, A. P. 2009, MNRAS, 392, 413

Stutz, A., Launhardt, R., Linz, H., et al. 2010, A\&A, 518, L87

Tachihara, K., Dobashi, K., Mizuno, A., Ogawa, H., \& Fukui, Y. 1996, PASJ, 48, 489

Tamura, Y., Kawabe, R., Shimajiri, Y., et al. 2015, ApJ, 808, 121

Testi, L., Natta, A., Scholz, A., et al. 2016, A\&A, 593, A111

Tobin, J. J., Looney, L. W., Li, Z.-Y., et al. 2016, ApJ, 818, 73

Tokuda, K., Tachihara, K., Saigo, K., et al. 2019, PASJ, 71, 73

Tsukagoshi, T., Saito, M., Kitamura, Y., et al. 2011, ApJ, 726, 45

van der Plas, G., Ménard, F., Ward-Duong, K., et al. 2016, ApJ, 819, 102

van Terwisga, S. E., van Dishoeck, E. F., Ansdell, M., et al. 2018, A\&A, 616, A88

Vázquez-Semadeni, E., Palau, A., Ballesteros-Paredes, J., Gómez, G. C., \& Zamora-Avilés, M. 2019, MNRAS, 490, 3061

Voirin, J., Manara, C. F., \& Prusti, T. 2018, A\&A, 610, A64

Ward-Duong, K., Patience, J., Bulger, J., et al. 2018, AJ, 155, 54

Ward-Thompson, D., Kirk, J. M., André, P., et al. 2010, A\&A, 518, L92

Whelan, E. T., Ray, T. P., Bacciotti, F., et al. 2005, Nature, 435, 652

Whelan, E. T., Riaz, B., \& Rouzé, B. 2018, A\&A, 610, L19

White, R. J., \& Basri, G. 2003, ApJ, 582, 1109

Whitworth, A. P., \& Zinnecker, H. 2004, A\&A, 427, 299

Williams, J. P., de Geus, E. J., \& Blitz, L. 1994, ApJ, 428, 693

Wright, E. L., Eisenhardt, P. R. M., Mainzer, A. K., et al. 2010, AJ, 140, 1868

Wu, Y.-L., Close, L. M., Males, J. R., et al. 2015, ApJ, 807, L13

Young, C. H., \& Evans, N. J., II 2005, ApJ, 627, 293 


\section{Appendix A: ALMA detections and non detections}

List of the ALMA pointings based on the AzTEC detections. The rms is measured at the phase centre except for the sources with ALMA detections. We included the previous classification based on the AzTEC detections and the SED from the SOLA catalog. Class II and Class I/II sources were obtained from the literature.

Table A.1. ALMA pointings including detections and non-detections.

\begin{tabular}{|c|c|c|c|c|c|}
\hline Name ${ }^{(1)}$ & RA (J2000) & $\operatorname{Dec}(\mathbf{J} 2000)^{(2)}$ & $\mathrm{rms}^{(3)}\left[\mathrm{mJy}\right.$ beam $\left.^{-1}\right]$ & Classification $^{(4)}$ & References ${ }^{(5)}$ \\
\hline $153701.1-332255$ & $15: 37: 01.10$ & $-33: 22: 55.00$ & 0.10 & Class II & 1 \\
\hline ALMA J153702.653-331924.92 ${ }^{(6)}$ & $15: 37: 03.10$ & -33.19 .27 .00 & 0.096 & Class II & 1,3 \\
\hline $153709.9-330129$ & 15:37:09.90 & $-33: 01: 29.00$ & 0.085 & Class II & 1 \\
\hline AzTEC-lup1-99 & $15: 38: 04.40$ & $-34: 52: 28.24$ & 0.054 & Starless core & \\
\hline AzTEC-lup1-103 & $15: 38: 12.90$ & $-34: 56: 23.70$ & 0.056 & Starless core & \\
\hline AzTEC-lup1-109 & $15: 38: 27.44$ & $-35: 12: 40.90$ & 0.057 & Starless core & \\
\hline AzTEC-lup1-72 & $15: 38: 46.93$ & $-33: 23: 36.38$ & 0.092 & Class I & \\
\hline AzTEC-lup1-111 & $15: 38: 59.73$ & $-33: 29: 50.16$ & 0.055 & Starless core & \\
\hline AzTEC-lup1-57 & $15: 39: 04.25$ & $-35: 06: 43.0$ & 0.069 & Starless core & \\
\hline ALMA J153914.996-332907.62 ${ }^{(6)}$ & $15: 39: 15.84$ & $-33: 28: 58.50$ & 0.094 & Starless core & \\
\hline $153921.8-340020$ & $15: 39: 21.80$ & $-34: 00: 20.00$ & 0.085 & Class II & 1 \\
\hline AzTEC-lup1-67 & $15: 39: 21.17$ & $-34: 43: 37.52$ & 0.071 & Starless core & \\
\hline AzTEC-lup1-114 & $15: 39: 49.35$ & $-34: 49: 26.22$ & 0.063 & Starless core & \\
\hline AzTEC-lup1-84 & $15: 40: 09.15$ & $-33: 32: 20.16$ & 0.057 & Starless core & \\
\hline AzTEC lup1-90 & $15: 40: 18.86$ & $-33: 41: 00.09$ & 0.061 & Class 0 & \\
\hline AzTEC-lup1-40 & $15: 40: 46.65$ & $-33: 43: 17.88$ & 0.10 & Class I & \\
\hline AzTEC-lup1-104 & $15: 41: 15.39$ & $-33: 46: 41.34$ & 0.057 & Starless core & \\
\hline AzTEC-lup1-101 & $15: 41: 28.04$ & $-33: 41: 51.37$ & 0.059 & Starless core & \\
\hline $154140.8-334519$ & $15: 41: 40.80$ & $-33: 45: 19.00$ & 0.089 & Class II & 1,2 \\
\hline AzTEC-lup1-119 & $15: 42: 05.21$ & $-33: 45: 59.71$ & 0.058 & Starless core & \\
\hline ALMA J154229.778-334241.86(6) & $15: 42: 29.56$ & $-33: 42: 39.94$ & 0.11 & Class 0 & \\
\hline AzTEC-lup1-124 & $15: 42: 38.60$ & $-33: 48: 52.24$ & 0.055 & Starless core & \\
\hline AzTEC-lup1-52 & $15: 42: 45.21$ & $-33: 58: 43.41$ & 0.065 & Starless core & \\
\hline AzTEC-lup1-54 & $15: 42: 45.02$ & $-34: 12: 01.36$ & 0.075 & Starless core & \\
\hline AzTEC-lup1-94 & $15: 43: 50.32$ & $-34: 01: 59.60$ & 0.067 & Starless core & \\
\hline $154433.9-335254$ & $15: 44: 33.90$ & $-33: 52: 54.00$ & 0.11 & Class II & 1 \\
\hline ALMA J154456.522-342532.99 (6) & $15: 44: 57.22$ & $-34: 25: 31.55$ & 0.068 & Starless core & \\
\hline AzTEC-lup1-71 & $15: 44: 59.34$ & $-34: 20: 55.49$ & 0.091 & Class 0 & \\
\hline ALMA J154506.515-344326.15 ${ }^{(6)}$ & $15: 45: 06.45$ & $-34: 43: 16.23$ & 0.089 & Starless core & \\
\hline AzTEC-lup1-123 & $15: 45: 40.24$ & $-35: 04: 56.61$ & 0.062 & Starless core & \\
\hline ALMA J154634.169-343301.90 ${ }^{(6)}$ & $15: 46: 33.47$ & -34.33 .05 .10 & 0.083 & Starless core & \\
\hline $160545.8-385454$ & $16: 05: 45.80$ & $-38: 54: 54.00$ & 0.062 & Class II & 1 \\
\hline ALMA J160658.604-390407.88 ${ }^{(6)}$ & $16: 06: 58.70$ & $-39: 04: 05.00$ & 0.062 & Class II & 1 \\
\hline AzTEC-lup-3-15 & $16: 07: 51.70$ & $-39: 07: 29.50$ & 0.058 & Starless core & \\
\hline $160714.0-385238$ & 16:07:14.00 & $-38: 52: 38.00$ & 0.060 & Class II & 1,2 \\
\hline ALMA J160804.168-390452.84 & 16:08:04.80 & $-39: 04: 49.00$ & 0.093 & Class II & 1,3 \\
\hline AzTEC-lup-3-20 & 16:08:14.40 & $-39: 10: 50.89$ & 0.067 & Starless core & \\
\hline $160816.0-390304$ & $16: 08: 16.00$ & $-39: 03: 04.00$ & 0.057 & Class II & $1,2,3$ \\
\hline $160826.8-384101^{(6)}$ & $16: 08: 26.80$ & $-38: 41: 01.00$ & 0.059 & Class II & 1 \\
\hline AzTEC-lup3-12 & $16: 08: 32.70$ & $-39: 04: 39.80$ & 0.061 & Starless core & \\
\hline $160833.0-385222$ & $16: 08: 33.05$ & $-38: 52: 22.40$ & 0.066 & Class II & 1,3 \\
\hline $160835.5-390035$ & $16: 08: 35.48$ & $-39: 00: 35.80$ & 0.067 & Class II & 1,3 \\
\hline Lup $706^{(6)}$ & $16: 08: 37.33$ & $-39: 23: 10.90$ & 0.090 & Class II & 1,3 \\
\hline AzTEC-lup3-10 & $16: 08: 41.60$ & $-39: 05: 23.91$ & 0.058 & Starless core & \\
\hline $160848.2-390920$ & $16: 08: 48.20$ & $-39: 09: 19.00$ & 0.051 & Class II & $1,2,3$ \\
\hline AzTEC-lup3-5 & $16: 08: 48.50$ & $-39: 07: 27.97$ & 0.066 & Starless core & \\
\hline Par-Lup3-4 ${ }^{(6)}$ & $16: 08: 51.44$ & $-39: 05: 30.50$ & 0.031 & Class I/II & $1,2,3$ \\
\hline AzTEC-lup-3-14 & $16: 08: 54.60$ & $-39: 12: 26.90$ & 0.052 & Starless core & \\
\hline AzTEC-lup-3-19 & $16: 08: 55.40$ & $-39: 05: 59.89$ & 0.060 & Starless core & \\
\hline SONYC-Lup3-7 ${ }^{(6)}$ & $16: 08: 59.53$ & $-38: 56: 27.80$ & 0.055 & Class I/II & $1,2,3$ \\
\hline AzTEC-lup3-4 & $16: 09: 13.60$ & $-39: 07: 43.95$ & 0.059 & Starless core & \\
\hline SONYC-lup3-10 & $16: 09: 13.43$ & $-38: 58: 04.90$ & 0.062 & Class I/II & 3 \\
\hline AzTEC-lup3-8 & 16:09:14.30 & $-39: 05: 23.95$ & 0.065 & Starless core & \\
\hline AzTEC-lup3-13 & $16: 09: 36.60$ & $-39: 03: 59.64$ & 0.069 & Starless core & \\
\hline ALMA J160920.171-384456.40 ${ }^{(6)}$ & 16:09:20.80 & -38.45 .10 .00 & 0.18 & Class II & 1,3 \\
\hline ALMA J160932.167-390832.27 ${ }^{(6)}$ & $16: 09: 32.80$ & $-39: 08: 44.11$ & 0.13 & Starless core & \\
\hline AzTEC-lup3-29 & $16: 10: 01.33$ & $-39: 06: 45.10$ & 0.057 & Class II & 1,2 \\
\hline AzTEC-lup3-9 & $16: 10: 05.90$ & $-39: 10: 54.84$ & 0.061 & Starless core & \\
\hline AzTEC-lup-3-21 & $16: 10: 08.22$ & $-39: 02: 51.68$ & 0.053 & Class I & \\
\hline AzTEC-lup-3-16 & $16: 10: 19.80$ & $-39: 11: 51.07$ & 0.067 & Starless core & \\
\hline ALMA J161030.273-383154.52 ${ }^{(6)}$ & $16: 10: 30.60$ & $-38: 31: 51.00$ & 0.064 & Class II & 1 \\
\hline $161144.9-383234$ & $16: 11: 44.88$ & $-38: 32: 44.90$ & 0.059 & Class II & $1,2,3$ \\
\hline $161225.6-381742$ & $16: 12: 25.60$ & $-38: 17: 42.00$ & 0.056 & Class II & 1,3 \\
\hline $161210.4-390904$ & $16: 12: 10.46$ & $-39: 09: 04.00$ & 0.051 & Class II & 1,3 \\
\hline
\end{tabular}

Notes. ${ }^{(1)}$ Name as it appears in the literature. AzTEC-lup is a denomination for the non ALMA detection pointings based on AzTEC detections. ${ }^{(2)}$ Phase center of the observation. ${ }^{(3)} \mathrm{rms}$ is measured at the phase center, except for the ALMA detected sources. ${ }^{(4)}$ Previous classification before the ALMA observation based on AzTEC data and optical/infrared counterpart. ${ }^{(5)}$ Specific references used to obtain spectroscopically confirmed Class II sources in the ALMA pointing: (1) Comerón et al. (2009a), (2) Merín et al. (2008), (3) Mužić et al. (2014). ${ }^{(6)}$ Sources detected with ALMA. See Table 2. 


\section{Appendix B: Additional figures}
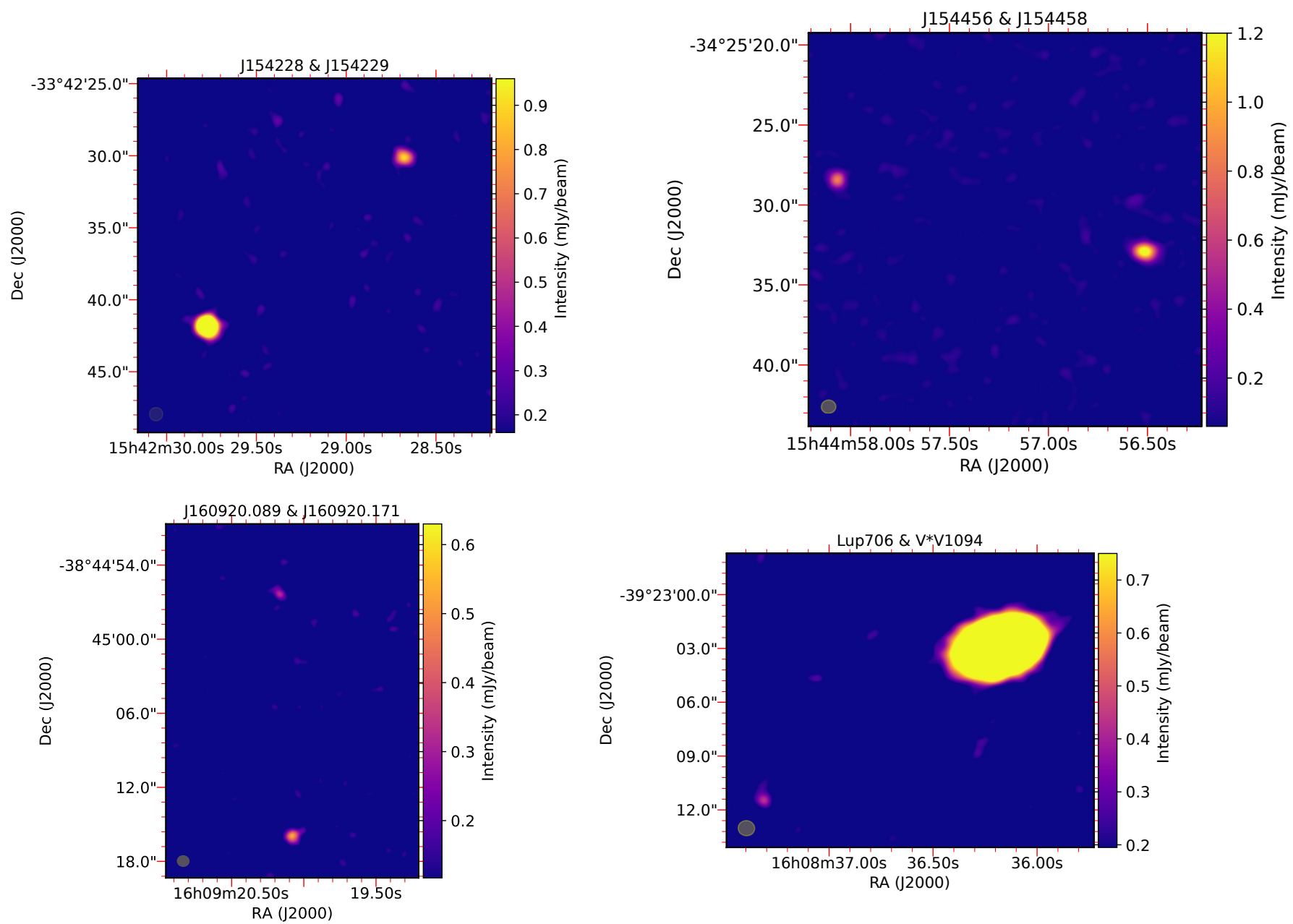

Fig. B.1. ALMA $1.3 \mathrm{~mm}$ maps for fields that show two sources inside the ALMA primary beam. Grey ellipse at the bottom-left corner represents the synthesized beam. Individual sources can be seen in more detail in Figs. 3 and B.2.
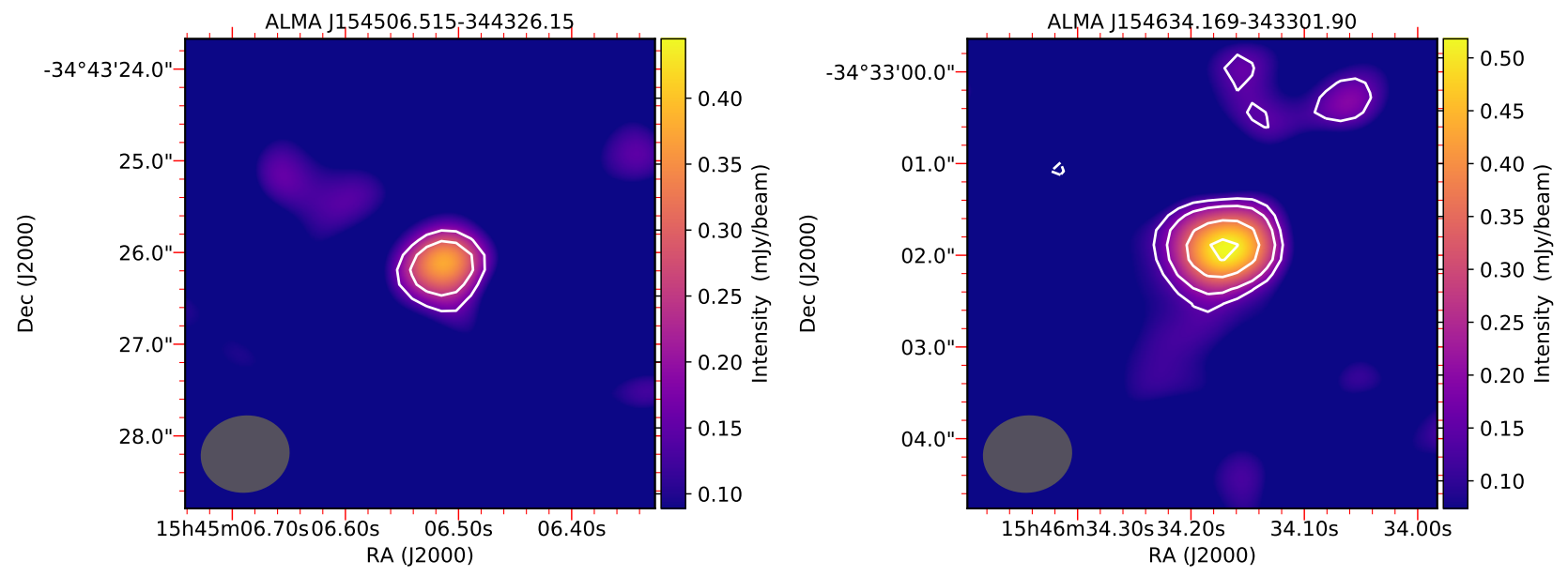

Fig. B.2. Continued from Fig. 3. 
A. Santamaría-Miranda et al.: Early stages of substellar formation in the Lupus 1 and 3 molecular clouds
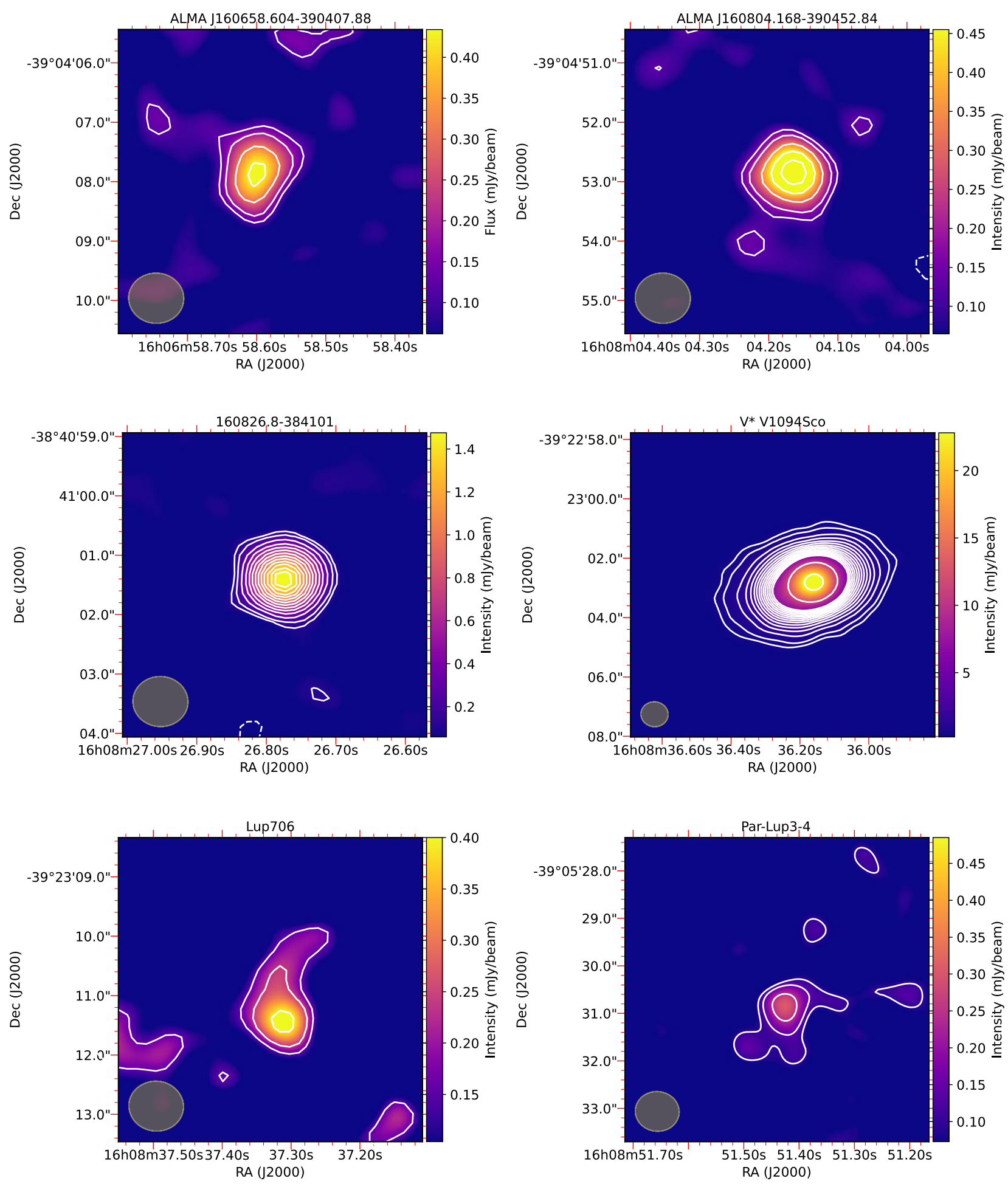

Fig. B.2. continued. 

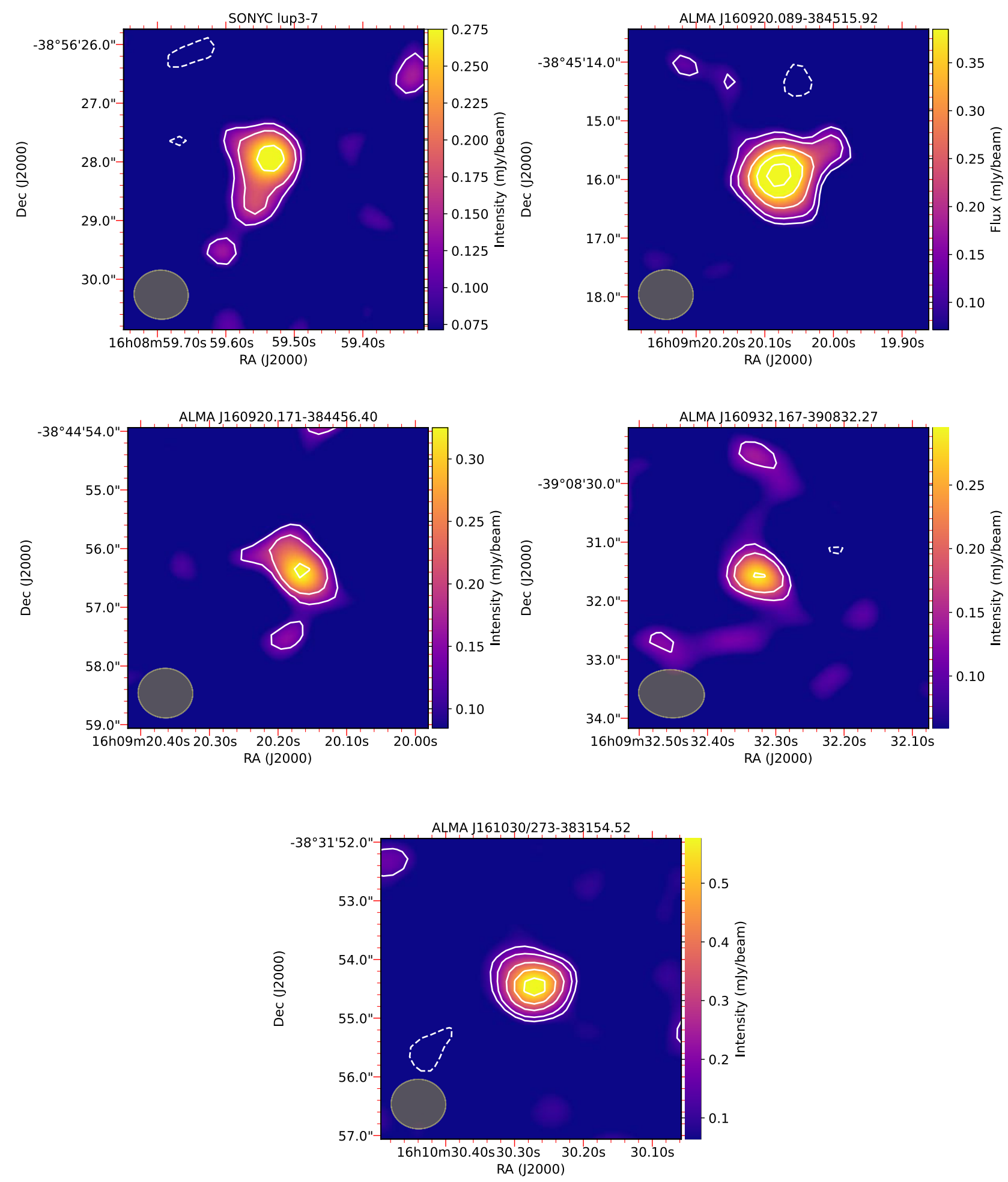

Fig. B.2. continued. 
A. Santamaría-Miranda et al.: Early stages of substellar formation in the Lupus 1 and 3 molecular clouds
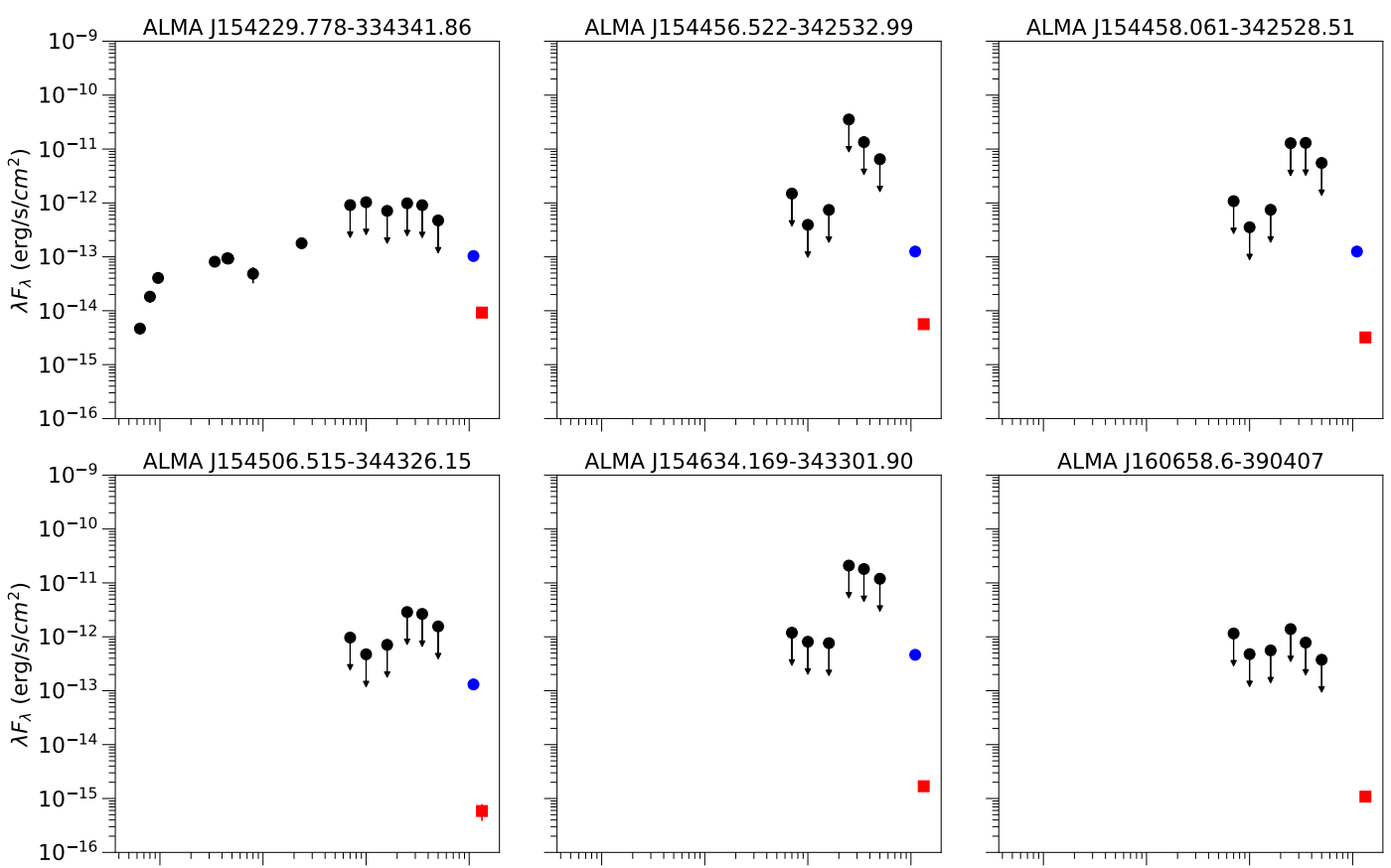

ALMA J154634.169-343301.90

ALMA J160658.6-390407
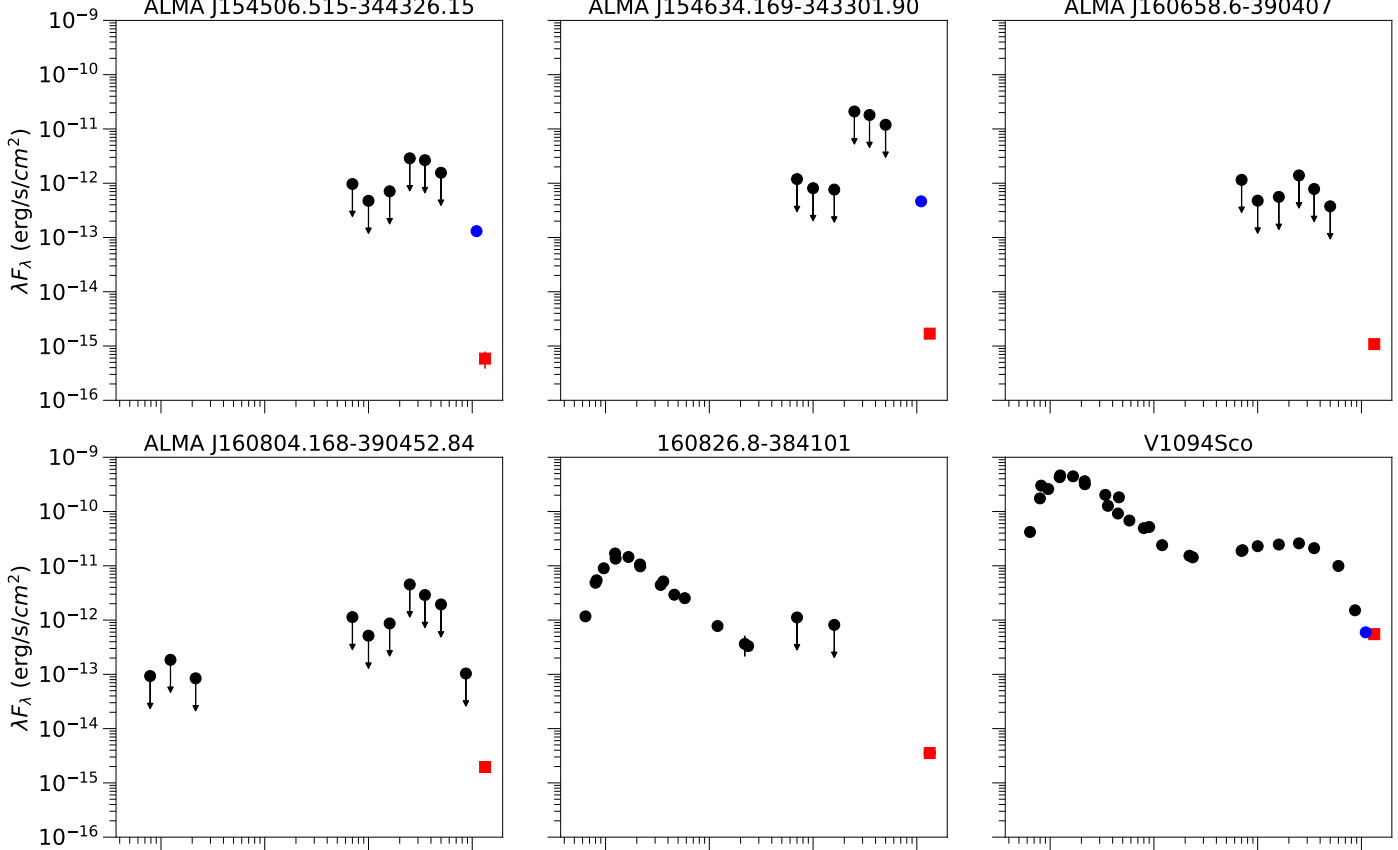

160826.8-384101

V1094Sco

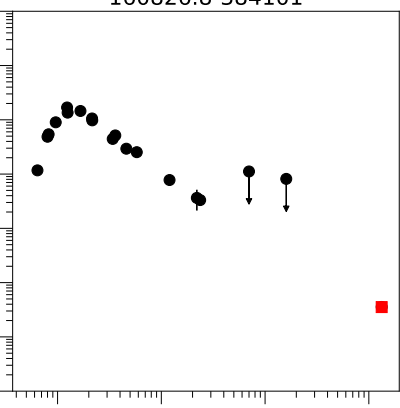

Par-lup3-4
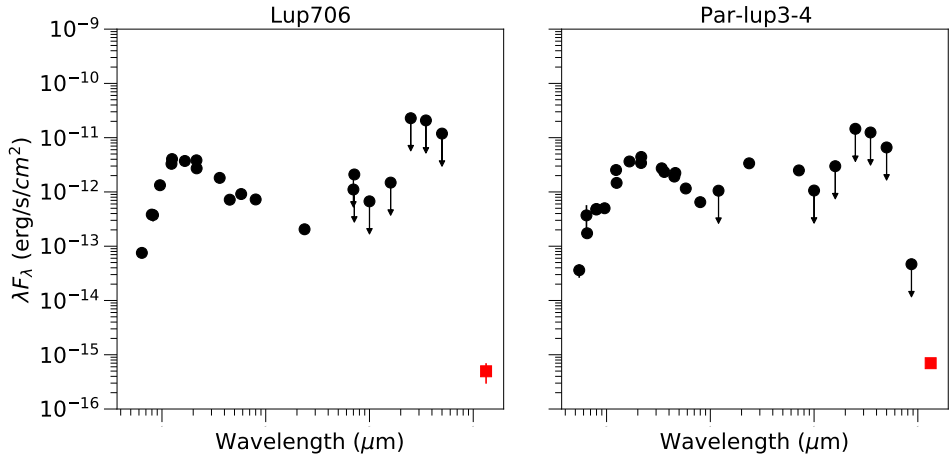

SONYC-lup3-7

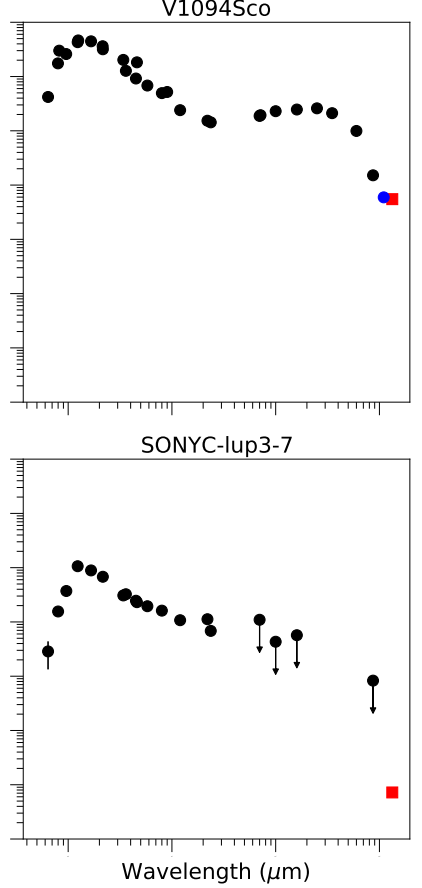

Wavelength $(\mu \mathrm{m})$

Fig. B.3. Continued from Fig. 4. 
A\&A 646, A10 (2021)
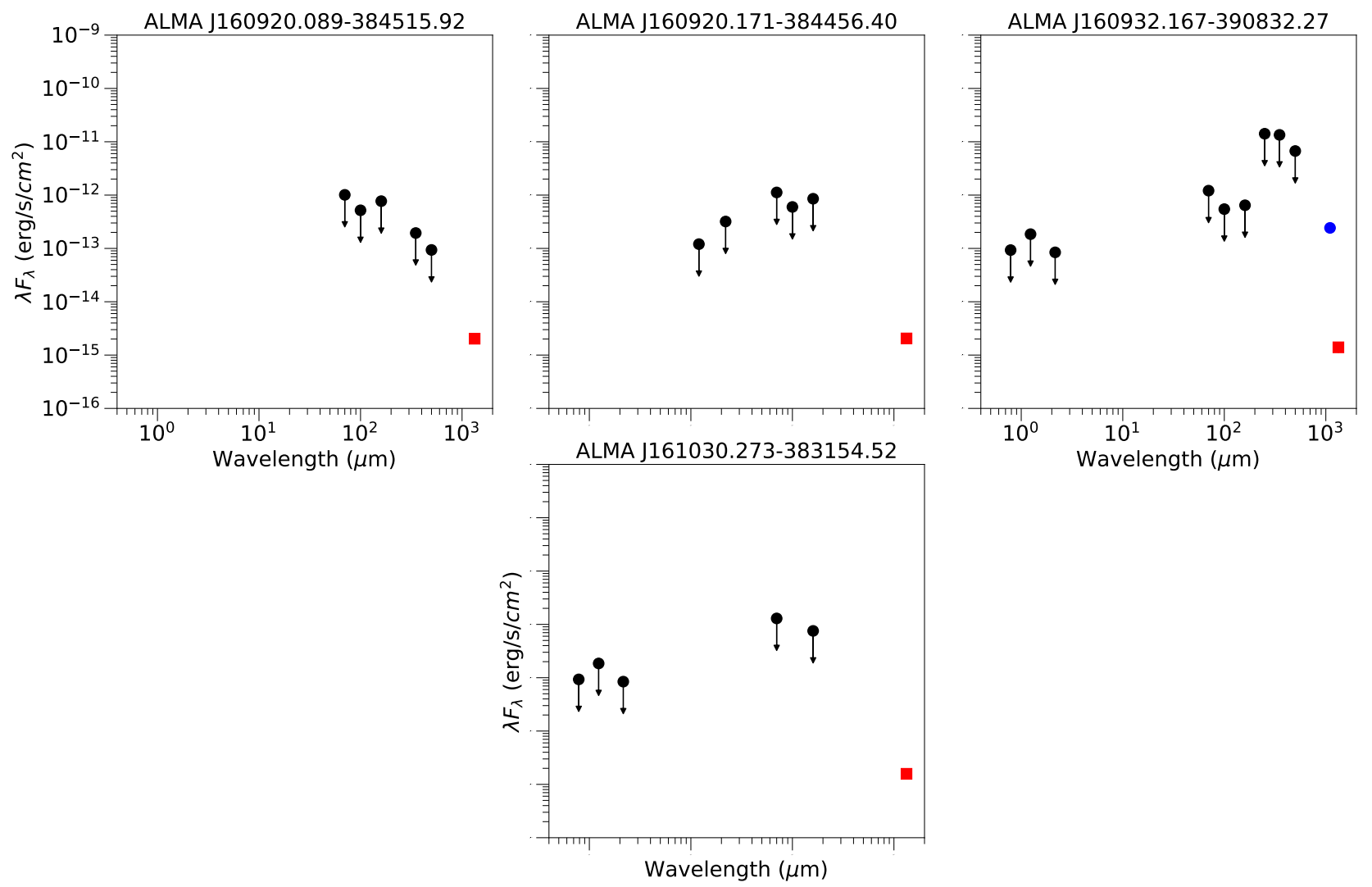

Fig. B.3. continued. 
A. Santamaría-Miranda et al.: Early stages of substellar formation in the Lupus 1 and 3 molecular clouds

ALMA J153914.996-332907.62

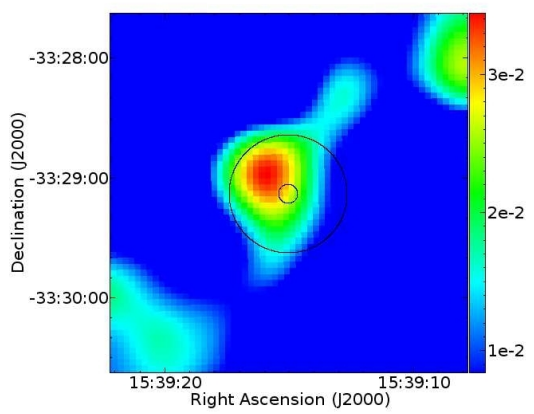

ALMA J154456.522-342532.99

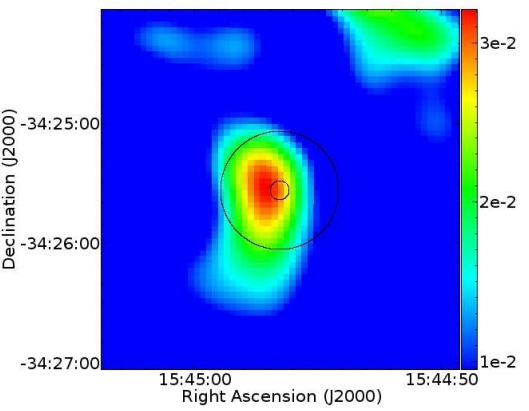

ALMA J154634.169-343301.90

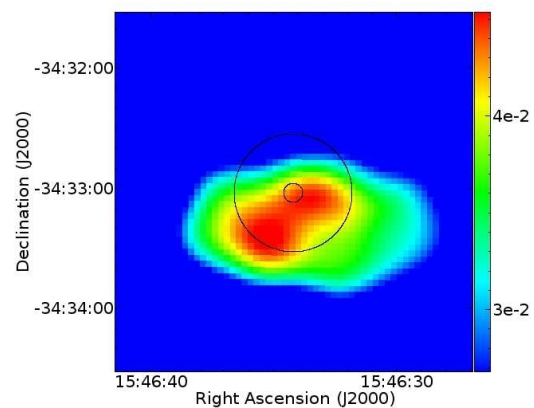

ALMA J160804.168-390452.84

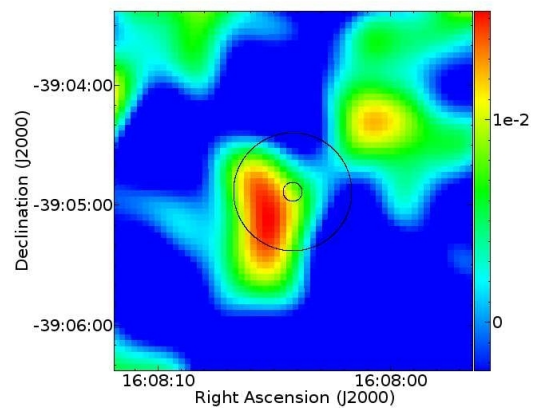

ALMA J154228.675-334230.18

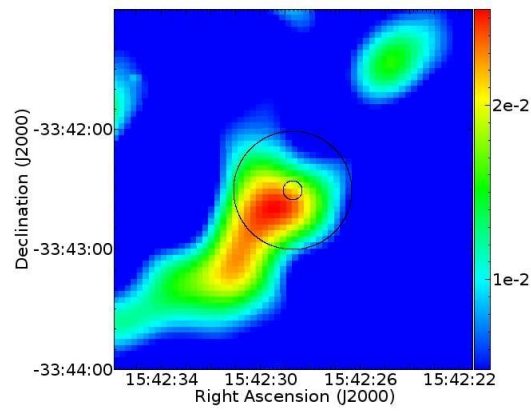

ALMA J154458.061-342528.41

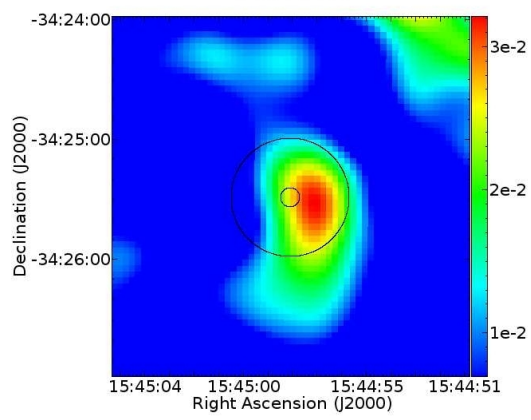

ALMA J160658.804-390407.89

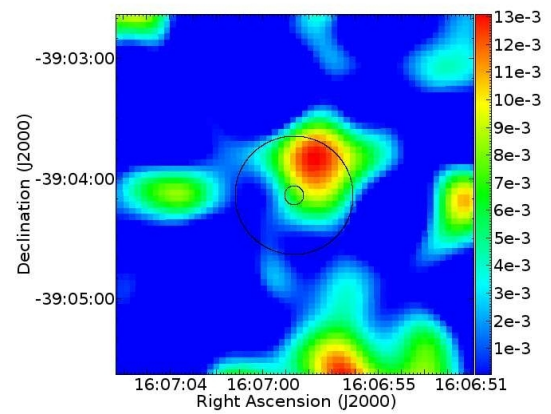

Lup706

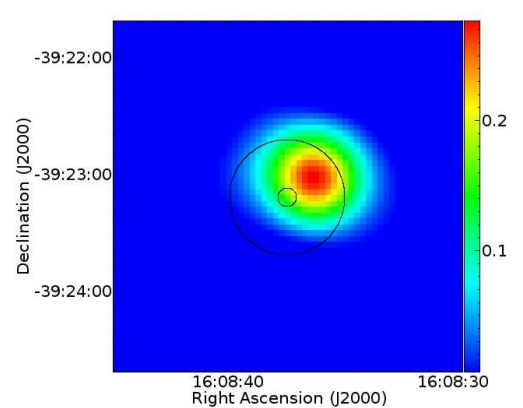

Fig. B.4. AzTEC maps centred at the position of the ALMA detections. The position is in RA and Dec (J2000). The wedge colour bar at the right shows the intensity $\left(\mathrm{mJy}_{\text {beam }}{ }^{-1}\right)$. The smaller circle is $5^{\prime \prime}$ in diameter and the greater one 30". SONYC-Lup3-7 and 161030.6-383151 have no images. 
A\&A 646, A10 (2021)

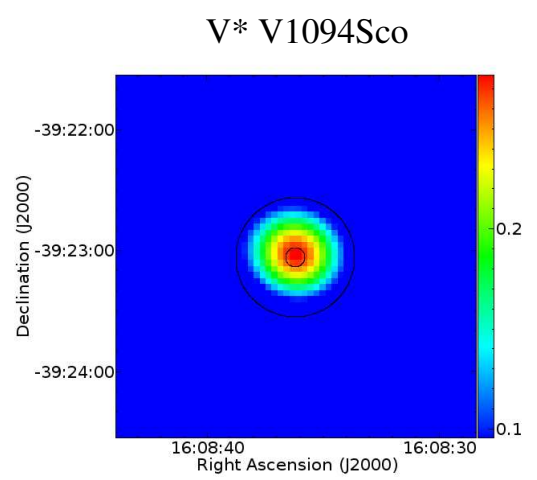

ALMA J160920.089-384515.92

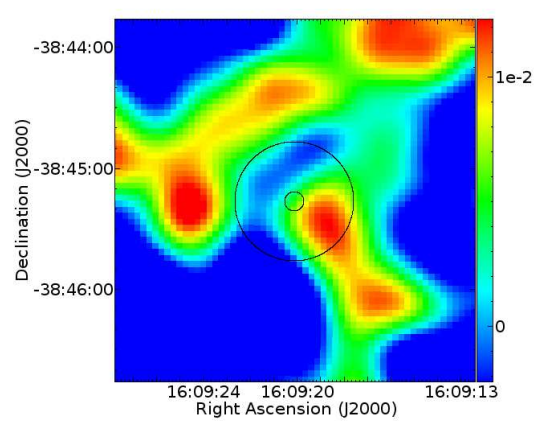

Fig. B.4. continued.

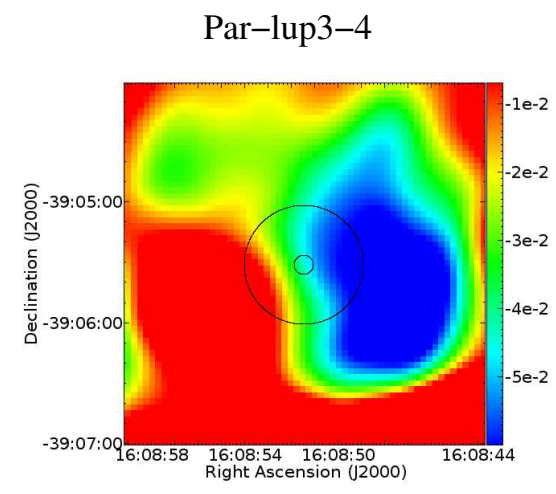

ALMA J160920.171-384456.40

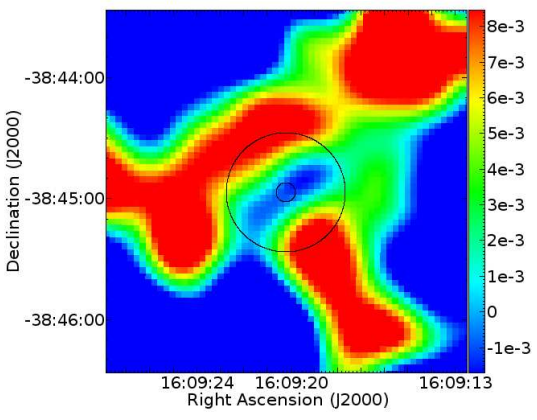

ALMA J160932.167-390832.27

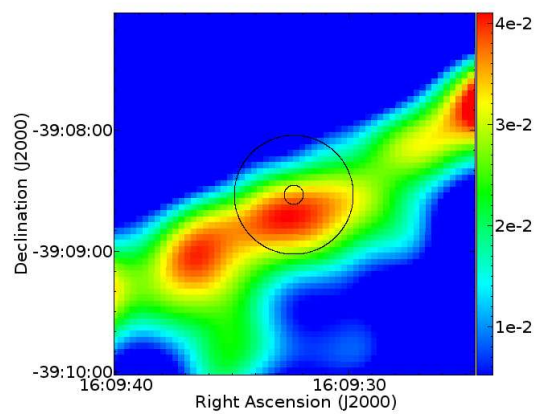


A. Santamaría-Miranda et al.: Early stages of substellar formation in the Lupus 1 and 3 molecular clouds

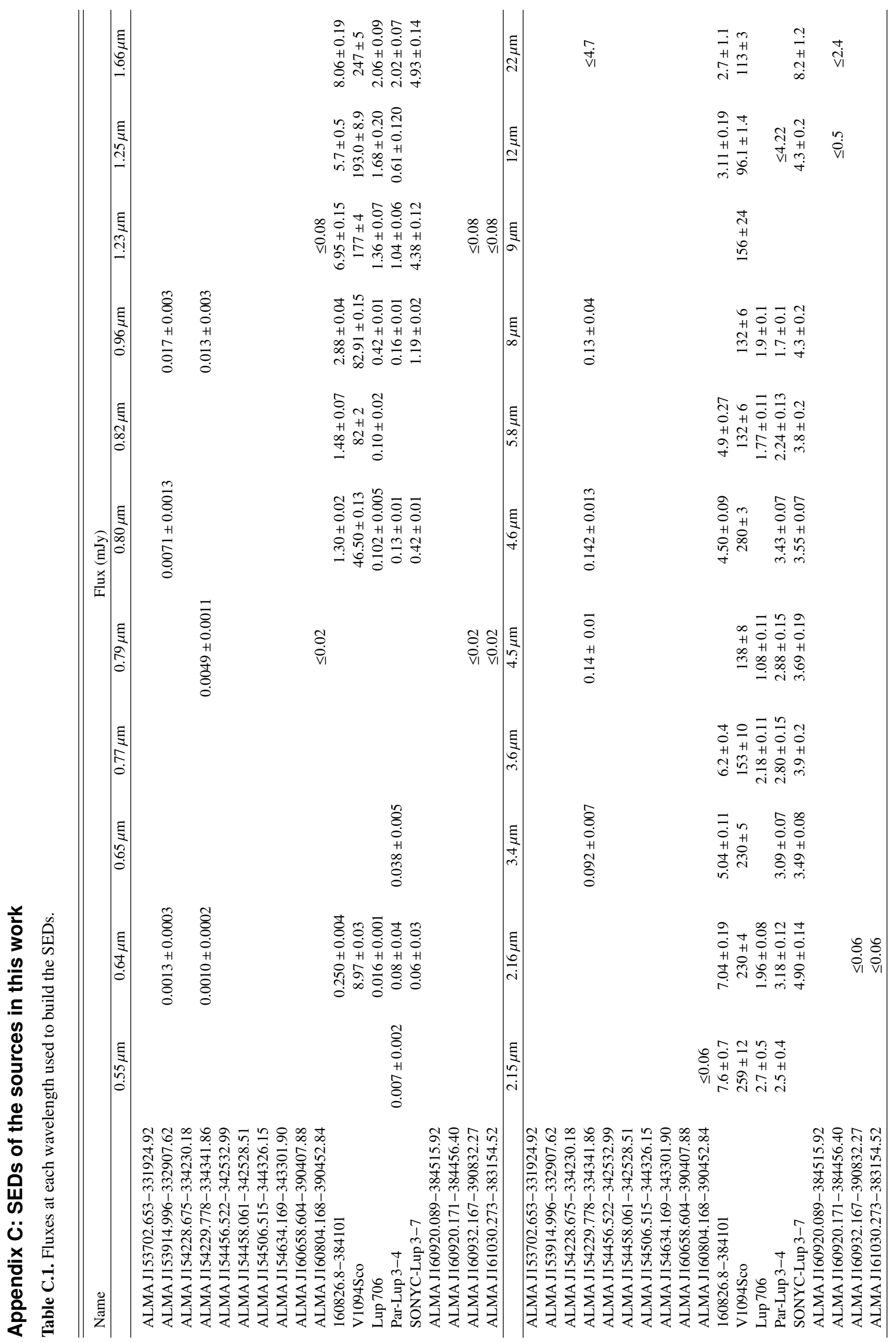




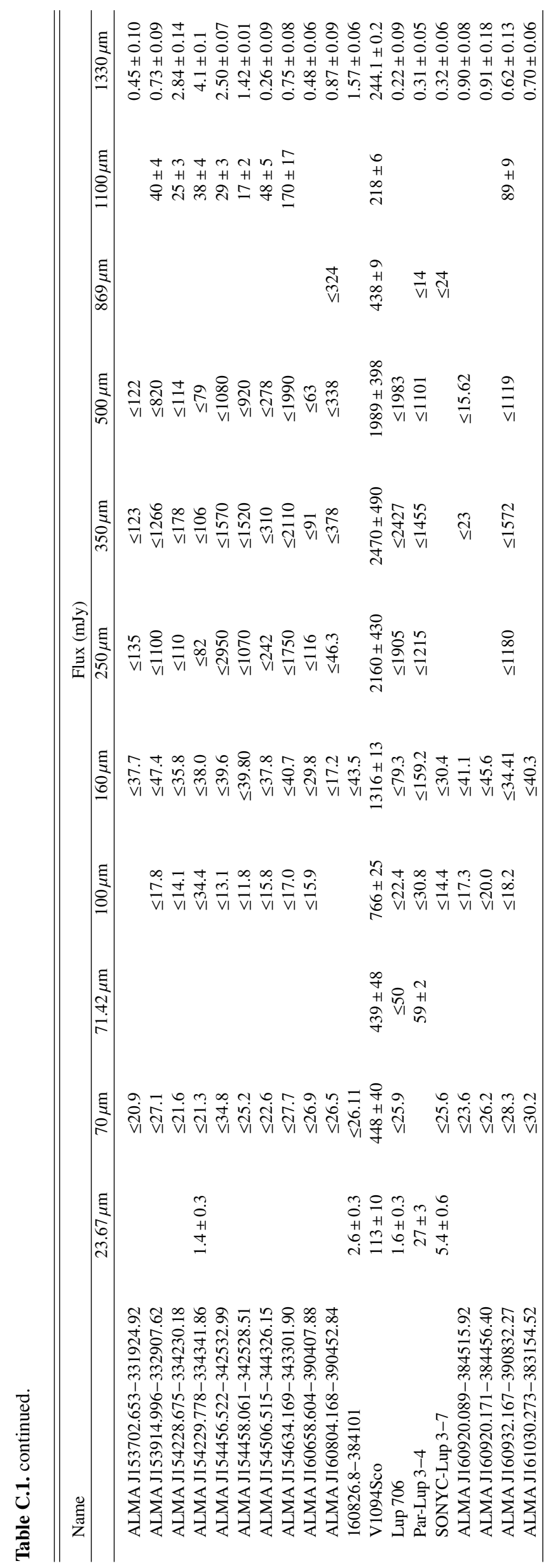

